Network Working Group

Request for Comments: 3665

BCP : 75

Category: Best Current Practice
A. Johnston

MCI

S. Donovan

R. Sparks

C. Cunningham dynamicsoft

K. Summers Sonus

December 2003

\title{
Session Initiation Protocol (SIP) Basic Call Flow Examples
}

Status of this Memo

This document specifies an Internet Best Current Practices for the Internet Community, and requests discussion and suggestions for improvements. Distribution of this memo is unlimited.

Copyright Notice

Copyright (C) The Internet Society (2003). All Rights Reserved.

Abstract

This document gives examples of Session Initiation Protocol (SIP)

call flows. Elements in these call flows include SIP User Agents and Clients, SIP Proxy and Redirect Servers. Scenarios include SIP

Registration and SIP session establishment. Call flow diagrams and message details are shown. 
Table of Contents

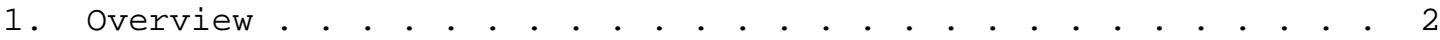

1.1. General Assumptions. . . . . . . . . . . . . . . . 3

1.2. Legend for Message Flows . . . . . . . . . . . . . . . 3

1.3. SIP Protocol Assumptions . . . . . . . . . . . . . . . . . . 4

2. SIP Registration . . . . . . . . . . . . . . . . . . . . . . 4

2.1. Successful New Registration. • • • • • • • • • • • • • 5

2.2. Update of Contact List . . . . . . . . . . . • . • . . 7

2.3. Request for Current Contact List. . . . . . . . . . 8

2.4. Cancellation of Registration . . . . . . . . . . . . . 9

2.5. Unsuccessful Registration. . . . . . . . . . . . . . . 10

3. SIP Session Establishment. • • • • • • • • • • • • . . . . 12

3.1. Successful Session Establishment . . . . . . . . . . 12

3.2. Session Establishment Through Two Proxies. . . . . . 15

3.3. Session with Multiple Proxy Authentication . . . . . . 26

3.4. Successful Session with Proxy Failure. • • • • • • • • 37

3.5. Session Through a SIP ALG. . . . . . . . . . . . . . 46

3.6. Session via Redirect and Proxy Servers with SDP in ACK . 54

3.7. Session with re-INVITE (IP Address Change) . . . . . . . 61

3.8. Unsuccessful No Answer . . . • . • . • • • • • • . 67

3.9. Unsuccessful Busy. . . . . . . . . . . . . . . . . . . . 75

3.10. Unsuccessful No Response from User Agent . . . . . . 80

3.11. Unsuccessful Temporarily Unavailable . • • • • • • • • 85

4. Security Considerations. . . . . . . . . . . . . . . . . . 91

5. References . . . . . . . . . . . . . . . . . . . . . 91

5.1. Normative References . . . . . . . . . . . . . . . 91

5.2. Informative References . . . . . . . . . . . . . . . . 91

6. Intellectual Property Statement. • • • • • • • • • • . . . . . 91

7. Acknowledgments. • • • • • • • • • • . . . . • . . . . 92

8. Authors' Addresses . . . . . . . . . . . . . . . . . . 93

9. Full Copyright Statement . . . . . . . . . . . . . . . 94

\section{Overview}

The call flows shown in this document were developed in the design of a SIP IP communications network. They represent an example minimum set of functionality.

It is the hope of the authors that this document will be useful for SIP implementers, designers, and protocol researchers alike and will help further the goal of a standard implementation of RFC 3261 [1]. These flows represent carefully checked and working group reviewed scenarios of the most basic examples as a companion to the specifications. 
These call flows are based on the current version 2.0 of SIP in RFC 3261 [1] with SDP usage described in RFC 3264 [2]. Other RFCs also comprise the SIP standard but are not used in this set of basic call flows.

Call flow examples of SIP interworking with the PSTN through gateways are contained in a companion document, $\mathrm{RFC} 3666$ [5].

The key words "MUST", "MUST NOT", "REQUIRED", "SHALL", "SHALL NOT", "SHOULD", "SHOULD NOT", "RECOMMENDED", "MAY", and "OPTIONAL" in this document are to be interpreted as described in BCP 14, RFC 2119 [4].

\subsection{General Assumptions}

A number of architecture, network, and protocol assumptions underlie the call flows in this document. Note that these assumptions are not requirements. They are outlined in this section so that they may be taken into consideration and to aid in the understanding of the call flow examples.

The authentication of SIP User Agents in these example call flows is performed using HTTP Digest as defined in [1] and [3].

Some Proxy Servers in these call flows insert Record-Route headers into requests to ensure that they are in the signaling path for future message exchanges.

These flows show TCP, TLS, and UDP for transport. See the discussion in RFC 3261 for details on the transport issues for SIP.

\subsection{Legend for Message Flows}

Dashed lines (---) represent signaling messages that are mandatory to the call scenario. These messages can be SIP or PSTN signaling. The arrow indicates the direction of message flow.

Double dashed lines $(===)$ represent media paths between network elements.

Messages with parentheses around their name represent optional messages.

Messages are identified in the Figures as F1, F2, etc. This references the message details in the list that follows the Figure. Comments in the message details are shown in the following form:

/* Comments. */ 


\subsection{SIP Protocol Assumptions}

This document does not prescribe the flows precisely as they are shown, but rather the flows illustrate the principles for best practice. They are best practices usages (orderings, syntax, selection of features for the purpose, handling of error) of SIP methods, headers and parameters. IMPORTANT: The exact flows here must not be copied as is by an implementer due to specific incorrect characteristics that were introduced into the document for convenience and are listed below. To sum up, the basic flows represent well-reviewed examples of SIP usage, which are best common practice according to IETF consensus.

For simplicity in reading and editing the document, there are a number of differences between some of the examples and actual SIP messages. For example, the HTTP Digest responses are not actual MD5 encodings. Call-IDs are often repeated, and CSeq counts often begin at 1. Header fields are usually shown in the same order. Usually only the minimum required header field set is shown, others that would normally be present such as Accept, supported, Allow, etc are not shown.

Actors:

Element Display Name UR

IP Address

$-------$

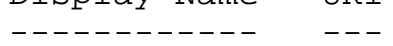

User Agent Alice

User Agent Bob

User Agent

Proxy Server

Proxy/Registrar

Proxy Server

ALG
alice@atlanta.example.com
$192 \cdot 0.2 .101$
bob@biloxi . example.com
boblchicago example.com
$192 \cdot 0.2 .201$
ss1.atlanta.example.com
ss2.biloxi example.com
ss3. chicago.example.com
192.0 .2 .100
$192 \cdot 0.2 .111$
192.0 .2 .222
192.0 .2 .233
alg1.atlanta.example.com

2. SIP Registration

Registration binds a particular device Contact URI with a SIP user Address of Record (AOR). 
2.1. Successful New Registration

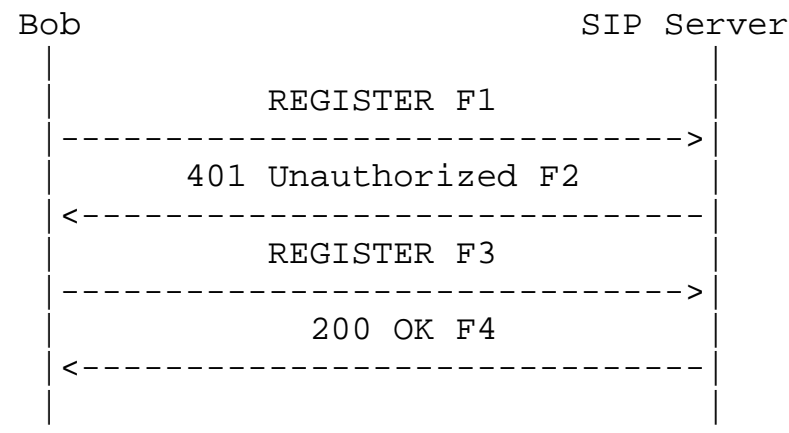

Bob sends a SIP REGISTER request to the SIP server. The request includes the user's contact list. This flow shows the use of HTTP Digest for authentication using TLS transport. TLS transport is used due to the lack of integrity protection in HTTP Digest and the danger of registration hijacking without it, as described in $\mathrm{RFC} 3261$ [1]. The SIP server provides a challenge to Bob. Bob enters her/his valid user ID and password. Bob's SIP client encrypts the user information according to the challenge issued by the SIP server and sends the response to the SIP server. The SIP server validates the user's credentials. It registers the user in its contact database and returns a response (200 OK) to Bob's SIP client. The response includes the user's current contact list in contact headers. The format of the authentication shown is HTTP digest. It is assumed that Bob has not previously registered with this server.

Message Details

F1 REGISTER Bob $->$ SIP Server

REGISTER sips:ss2.biloxi.example.com SIP/2.0

Via: SIP/2.0/TLS client.biloxi.example.com:5061; branch=z9hG4bKnashds 7 Max-Forwards: 70

From: Bob <sips:bobabiloxi.example.com>; tag=a73kszlfl

To: Bob <sips:bobebiloxi.example.com>

Call-ID: 1j9FpLxk3uxtm8tn@biloxi.example.com

CSeq: 1 REGISTER

Contact: <sips:bob@client.biloxi.example.com>

Content-Length: 0 
F2 401 Unauthorized SIP Server $->$ Bob

SIP/2.0 401 Unauthorized

Via: SIP/2.0/TLS client.biloxi.example.com:5061; branch=z9hG4bKnashds 7 i received=192.0.2.201

From: Bob <sips:bobabiloxi.example.com>; tag=a73kszlfl

To: Bob <sips:bob@biloxi.example.com>; tag=1410948204

Call-ID: 1 j9FpLxk3uxtm8tn@biloxi.example.com

CSeq: 1 REGISTER

WWW-Authenticate: Digest realm="atlanta.example.com", qop="auth", nonce="ea9c8e88df84f 1 cec4341ae6cbe5a359", opaque="", stale=FALSE, algorithm=MD5

Content-Length: 0

F3 REGISTER Bob $->$ SIP Server

REGISTER sips:ss2.biloxi.example.com SIP/2.0

Via: SIP/2.0/TLS client.biloxi.example.com:5061; branch=z9hG4bKnashd 92 Max-Forwards: 70

From: Bob <sips:bob@biloxi.example.com>; tag=ja743ks76zlflH

To: Bob <sips:bobebiloxi.example.com>

Call-ID: 1 j9FpLxk3uxtm8tn@biloxi.example.com

CSeq: 2 REGISTER

Contact: <sips:bob@client.biloxi.example.com>

Authorization: Digest username="bob", realm="atlanta.example.com" nonce="ea9c8e88df84f1cec4341ae6cbe5a359", opaque=" ", uri="sips:ss2.biloxi.example.com", response="dfe56131d1958046689d83306477ecc"

Content-Length: 0

F4 200 OK SIP Server $->$ Bob

$\mathrm{SIP} / 2.0200 \mathrm{OK}$

Via: SIP/2.0/TLS client.biloxi.example.com:5061; branch=z9hG4bKnashd 92 ; received=192.0.2.201

From: Bob <sips:boblbiloxi.example.com>; tag=ja743ks76zlflH

To: Bob <sips:bob@biloxi.example.com>; tag=37GkEhwl 6

Call-ID: 1 j9FpLxk3uxtm8tn@biloxi.example.com

CSeq: 2 REGISTER

Contact: <sips:bob@client.biloxi.example.com>; expires $=3600$

Content-Length: 0 
2.2. Update of Contact List

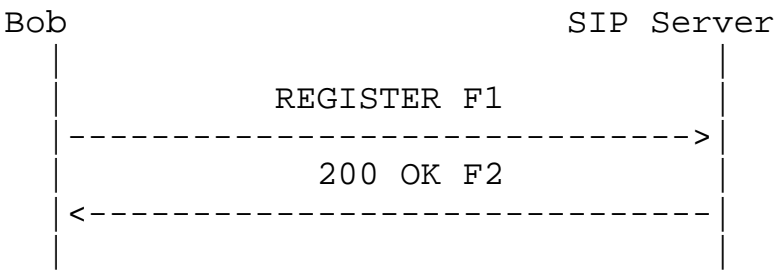

Bob wishes to update the list of addresses where the SIP server will redirect or forward INVITE requests.

Bob sends a SIP REGISTER request to the SIP server. Bob's request includes an updated contact list. Since the user already has authenticated with the server, the user supplies authentication credentials with the request and is not challenged by the server. The SIP server validates the user's credentials. It registers the user in its contact database, updates the user's contact list, and returns a response (200 OK) to Bob's SIP client. The response includes the user's current contact list in Contact headers.

Message Details

F1 REGISTER BOb $->$ SIP Server

REGISTER sips:ss2.biloxi.example.com SIP/2.0

Via: SIP/2.0/TLS client.biloxi.example.com:5061; branch=z9hG4bKnashds 7 Max-Forwards: 70

From: Bob <sips:bob@biloxi.example.com>; tag=a73kszlfl

To: Bob <sips:bob@biloxi.example.com>

Call-ID: 1 j9FpLxk3uxtm8tn@biloxi.example.com

CSeq: 1 REGISTER

Contact: mailto:bobebiloxi.example.com

Authorization: Digest username="bob", realm="atlanta.example.com", qop="auth", nonce="1cec4341ae6cbe5a359ea9c8e88df84f", opaque=" ", uri="sips:ss2.biloxi .example.com", response="71ba27c64bd01de719686aa4590d5824"

Content-Length: 0

F2 200 OK SIP Server $->$ Bob

SIP/2.0 200 OK

Via: SIP/2.0/TLS client.biloxi.example.com:5061; branch=z9hG4bKnashds 7 ; received=192.0.2.201

From: Bob <sips:bob@biloxi.example.com>; tag=a73kszlfl

To: Bob <sips:bob@biloxi.example.com>; tag $=34095828$ jh 
Call-ID: 1j9FpLxk3uxtm8tn@biloxi.example.com

CSeq: 1 REGISTER

Contact: <sips:bob@client.biloxi.example.com>; expires $=3600$

Contact: <mailto:bob@biloxi. example.com>; expires $=4294967295$

Content-Length: 0

2.3. Request for Current Contact List

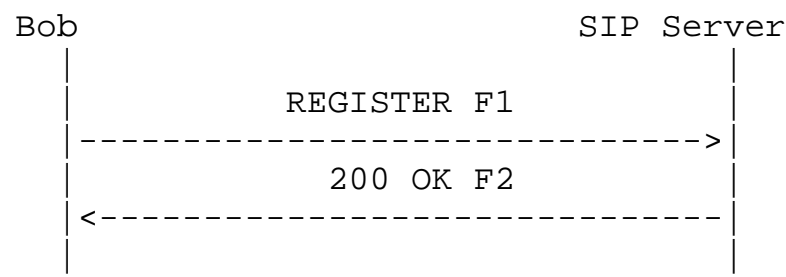

Bob sends a register request to the Proxy Server containing no Contact headers, indicating the user wishes to query the server for the user's current contact list. Since the user already has authenticated with the server, the user supplies authentication credentials with the request and is not challenged by the server. The SIP server validates the user's credentials. The server returns a response (200 OK) which includes the user's current registration list in contact headers.

Message Details

F1 REGISTER Bob $->$ SIP Server

REGISTER sips:ss2.biloxi.example.com SIP/2.0

Via: SIP/2.0/TLS client.biloxi.example.com:5061; branch=z9hG4bKnashds 7 Max-Forwards: 70

From: Bob <sips:bob@biloxi.example.com>; tag=a73kszlfl

To: Bob <sips:bobebiloxi.example.com>

Call-ID: 1 j9FpLxk3uxtm8tn@biloxi.example.com

CSeq: 1 REGISTER

Authorization: Digest username="bob", realm="atlanta.example.com", nonce="df84f1cec4341ae6cbe5ap359a9c8e88", opaque=" ", uri="sips:ss2.biloxi.example.com", response="aa7ab4678258377c6f7d4be6087e2f60"

Content-Length: 0

F2 200 OK SIP Server -> Bob

SIP $/ 2.0200$ OK

Via: SIP/2.0/TLS client.biloxi.example.com:5061; branch=z9hG4bKnashds 7 ; received=192.0.2.201 
From: Bob <sips:bob@biloxi. example.com>; tag=a73kszlfl

To: Bob <sips:bob@biloxi.example.com>; tag=jqoiweu75

Call-ID: 1j9FpLxk3uxtm8tn@biloxi.example.com

CSeq: 1 REGISTER

Contact: <sips:bobeclient.biloxi.example.com>; expires $=3600$

Contact: <mailto:bob@biloxi. example. com>; expires $=4294967295$

Content-Length: 0

2.4. Cancellation of Registration

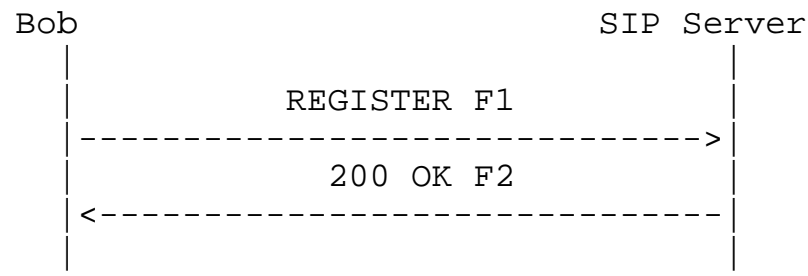

Bob wishes to cancel their registration with the SIP server. Bob sends a SIP REGISTER request to the SIP server. The request has an expiration period of 0 and applies to all existing contact locations. Since the user already has authenticated with the server, the user supplies authentication credentials with the request and is not challenged by the server. The SIP server validates the user's credentials. It clears the user's contact list, and returns a response (200 OK) to Bob's SIP client.

Message Details

F1 REGISTER Bob $->$ SIP Server

REGISTER sips:ss2.biloxi.example.com SIP/2.0

Via: SIP/2.0/TLS client.biloxi.example.com:5061; branch=z9hG4bKnashds 7 Max-Forwards: 70

From: Bob <sips:bob@biloxi.example.com>; tag=a73kszlfl

To: Bob <sips:bobebiloxi.example.com>

Call-ID: 1j9FpLxk3uxtm8tn@biloxi.example.com

CSeq: 1 REGISTER

Expires: 0

Contact: *

Authorization: Digest username="bob", realm="atlanta.example.com", nonce="88df84f1cac4341aea9c8ee6cbe5a359", opaque=" ", uri="sips:ss2.biloxi.example.com", response="ff0437c51696f9a76244f0cf1dbabbea"

Content-Length: 0

F2 200 OK SIP Server $\rightarrow$ Bob 
SIP $/ 2.0200$ OK

Via: SIP/2.0/TLS client.biloxi.example.com:5061; branch=z9hG4bKnashds 7 ; received=192.0.2.201

From: Bob <sips:bob@biloxi.example.com>; tag=a73kszlfl

To: Bob <sips:bob@biloxi.example.com>; tag=1418nmdsrf

Call-ID: 1 j9FpLxk3uxtm8tn@biloxi.example.com

CSeq: 1 REGISTER

Content-Length: 0

2.5. Unsuccessful Registration

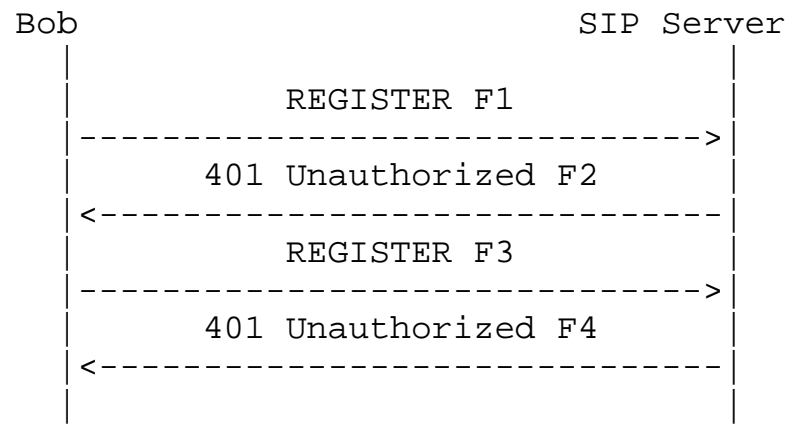

Bob sends a SIP REGISTER request to the SIP Server. The SIP server provides a challenge to Bob. Bob enters her/his user ID and password. Bob's SIP client encrypts the user information according to the challenge issued by the SIP server and sends the response to the SIP server. The SIP server attempts to validate the user's credentials, but they are not valid (the user's password does not match the password established for the user's account). The server returns a response (401 Unauthorized) to Bob's SIP client.

Message Details

F1 REGISTER Bob $->$ SIP Server

REGISTER sips:ss2.biloxi.example.com SIP/2.0

Via: SIP/2.0/TLS client.biloxi.example.com:5061; branch=z9hG4bKnashds 7 ; received=192.0.2.201

From: Bob <sips:bob@biloxi. example.com>; tag=a73kszlfl

To: Bob <sips:bob@biloxi.example.com>

Call-ID: 1j9FpLxk3uxtm8tn@biloxi.example.com

CSeq: 1 REGISTER

Contact: <sips:bob@client.biloxi.example.com>

Content-Length: 0 


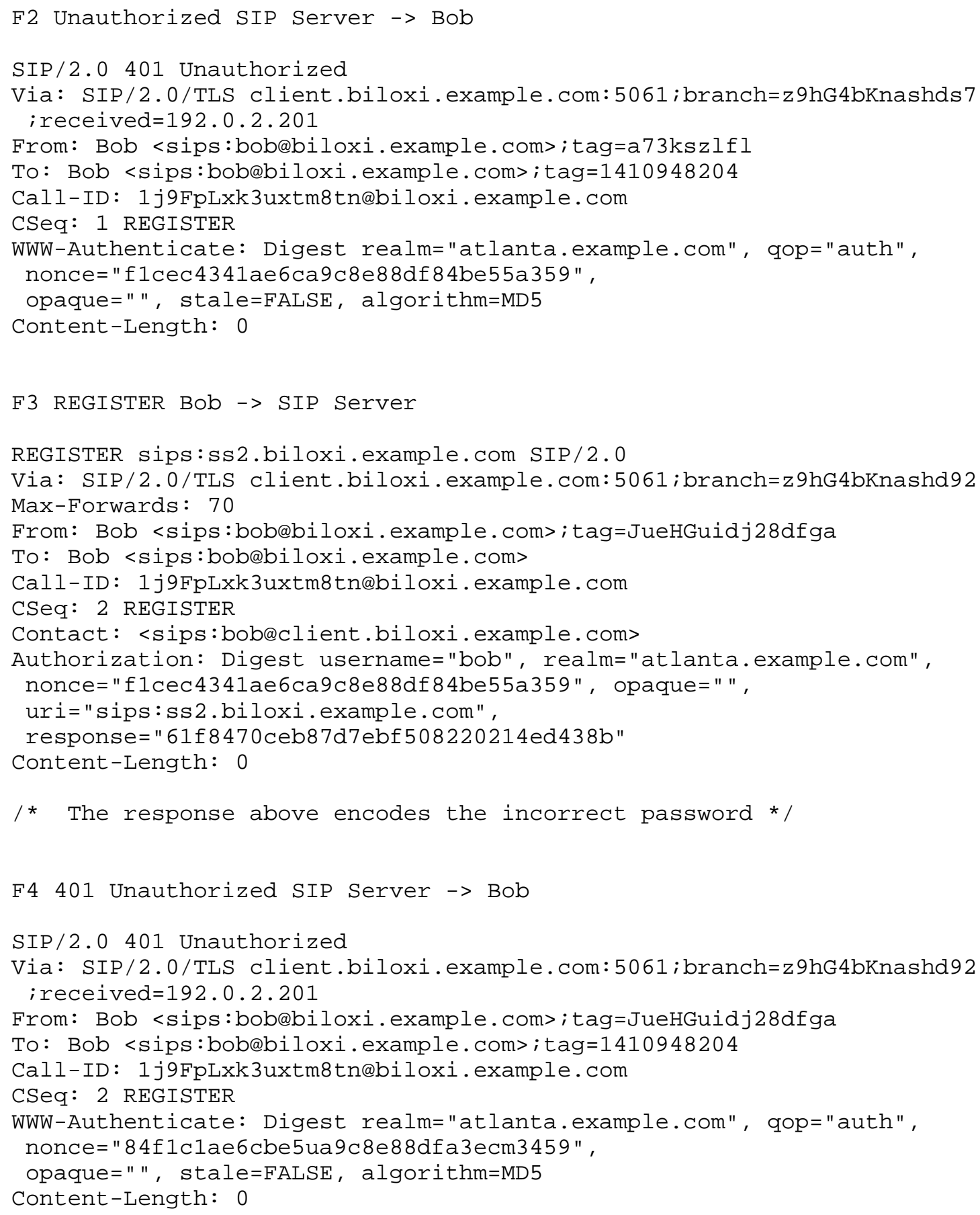


3. SIP Session Establishment

This section details session establishment between two SIP User Agents (UAs): Alice and Bob. Alice (sip:alicelatlanta.example.com) and Bob (sip:boblbiloxi.example.com) are assumed to be SIP phones or SIP-enabled devices. The successful calls show the initial signaling, the exchange of media information in the form of SDP payloads, the establishment of the media session, then finally the termination of the call.

HTTP Digest authentication is used by Proxy Servers to authenticate the caller Alice. It is assumed that Bob has registered with Proxy Server Proxy 2 as per section 2 to be able to receive the calls via the Proxy.

3.1. Successful Session Establishment

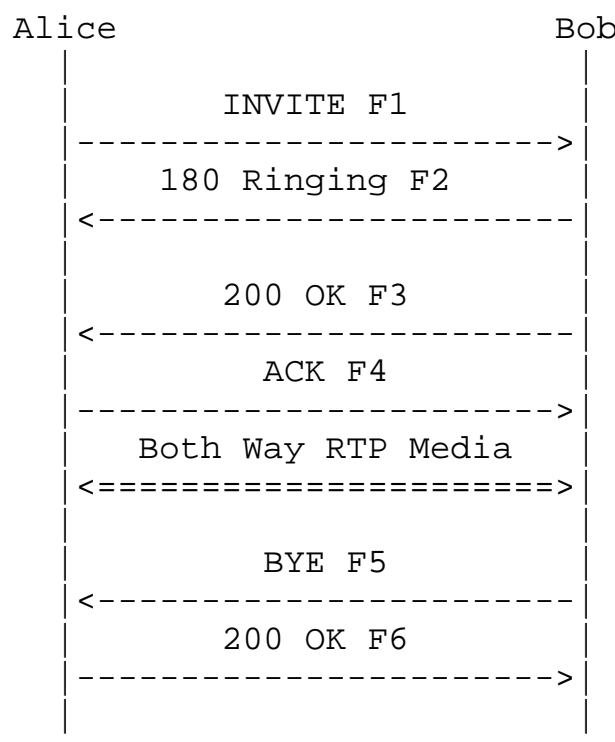

In this scenario, Alice completes a call to Bob directly.

Message Details

F1 INVITE Alice $->$ Bob

INVITE sip:bob@biloxi.example.com SIP/2.0

Via: SIP/2.0/TCP client.atlanta.example.com:5060; branch=z9hG4bK74bf 9 Max-Forwards: 70

From: Alice <sip:alicelatlanta.example.com>; tag=9fxced76sl

To: Bob <sip:bob@biloxi.example.com> 


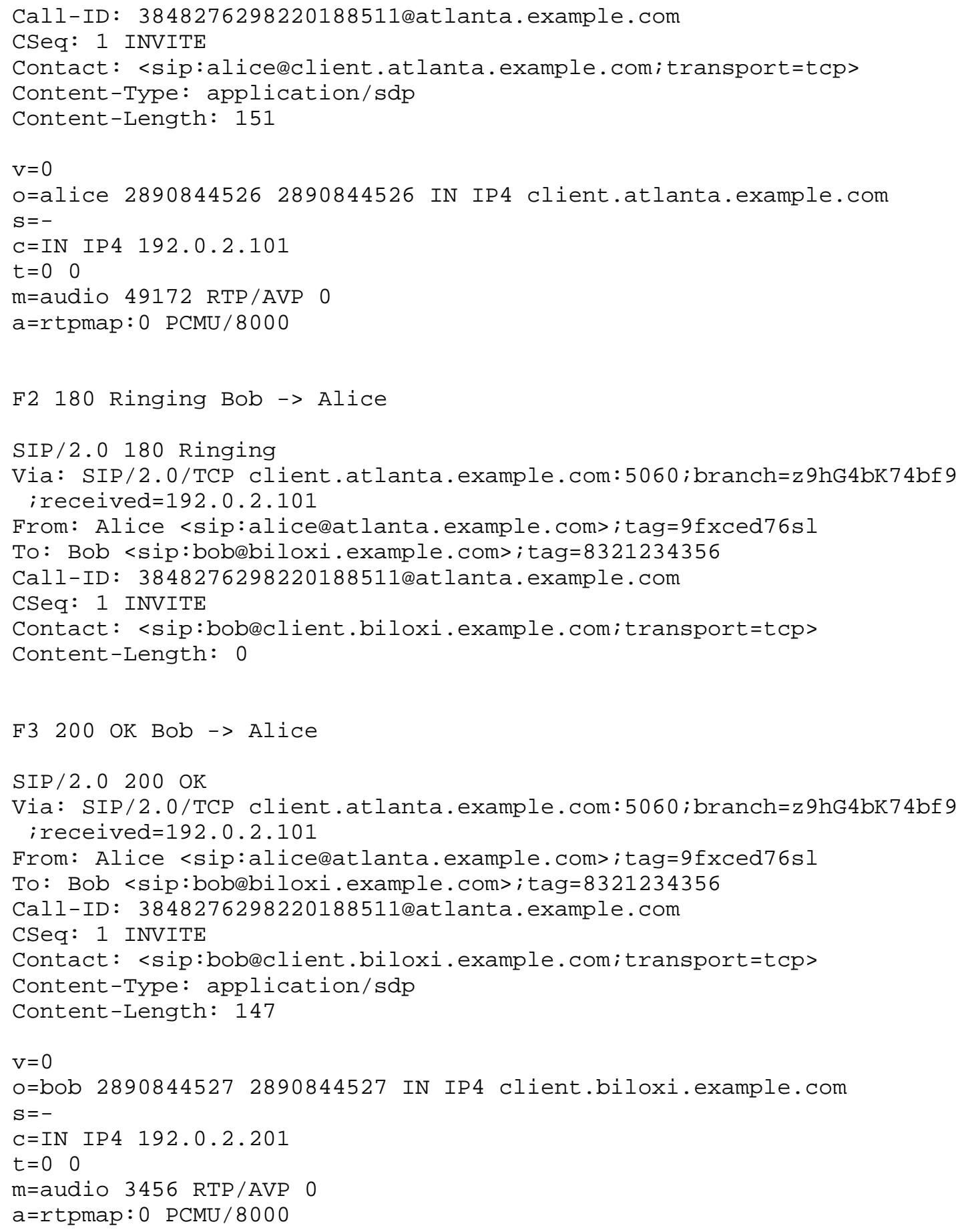




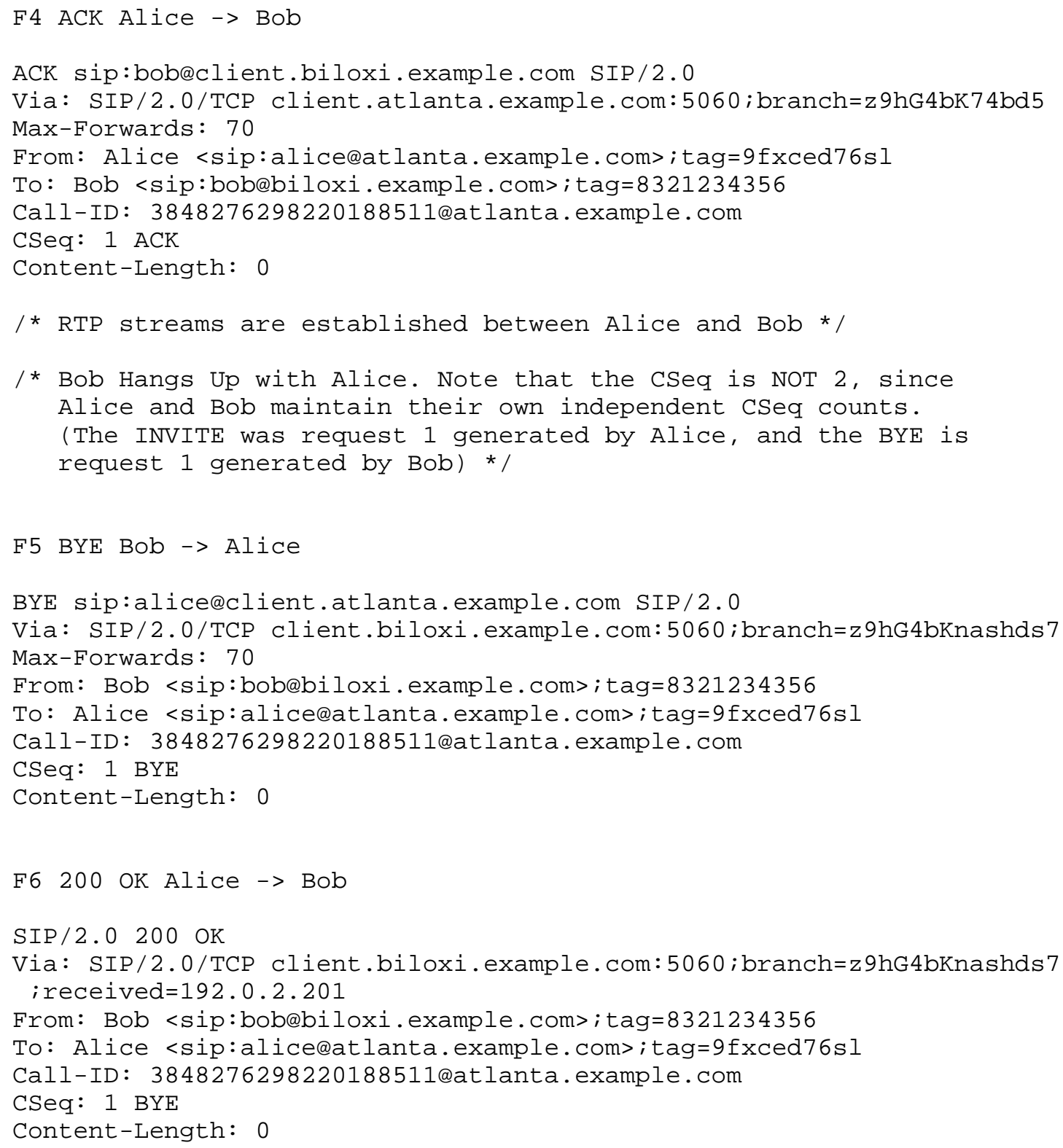




\subsection{Session Establishment Through Two Proxies}

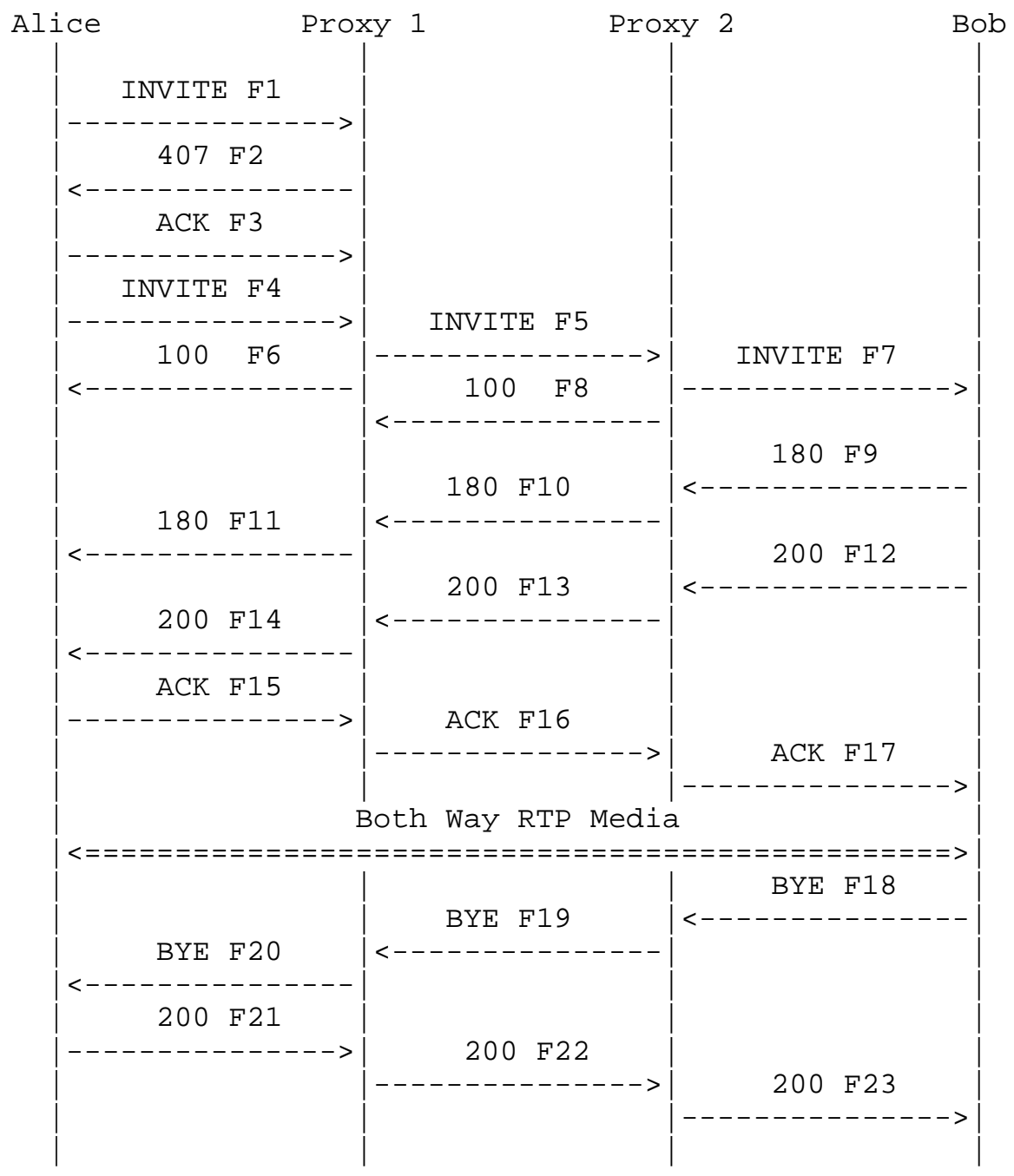

In this scenario, Alice completes a call to Bob using two proxies Proxy 1 and Proxy 2. The initial INVITE (F1) contains a pre-loaded Route header with the address of Proxy 1 (Proxy 1 is configured as a default outbound proxy for Alice). The request does not contain the Authorization credentials Proxy 1 requires, so a 407 Proxy Authorization response is sent containing the challenge information. A new INVITE (F4) is then sent containing the correct credentials and the call proceeds. The call terminates when Bob disconnects by initiating a BYE message. 
Proxy 1 inserts a Record-Route header into the INVITE message to ensure that it is present in all subsequent message exchanges. Proxy 2 also inserts itself into the Record-Route header. The ACK (F15) and BYE (F18) both have a Route header.

Message Details

F1 INVITE Alice $->$ Proxy 1

INVITE sip:bob@biloxi.example.com SIP/2.0

Via: SIP/2.0/TCP client. atlanta. example. com:5060; branch=z9hG4bK 74 b 43 Max-Forwards: 70

Route: <sip:ssl.atlanta.example.com;lr>

From: Alice <sip:alice@atlanta.example.com>; tag=9fxced76sl

To: Bob <sip:bobebiloxi.example.com>

Call-ID: 3848276298220188511 datlanta.example.com

CSeq: 1 INVITE

Contact: <sip:alicedclient.atlanta.example.com; transport=tcp>

Content-Type: application/sdp

Content-Length: 151

$\mathrm{V}=0$

o=alice 28908445262890844526 IN IP4 client.atlanta.example.com

$\mathrm{S}=-$

$\mathrm{C}=\mathrm{IN}$ IP 4 192.0.2.101

$t=0 \quad 0$

m=audio 49172 RTP/AVP 0

$\mathrm{a}=$ rtpmap $: 0 \mathrm{PCMU} / 8000$

/* Proxy 1 challenges Alice for authentication */

F2 407 Proxy Authorization Required Proxy 1 -> Alice

SIP/2.0 407 Proxy Authorization Required

Via: SIP/2.0/TCP client.atlanta.example.com:5060; branch=z9hG4bK74b43 ; received=192.0.2.101

From: Alice <sip:alicelatlanta.example.com>; tag=9fxced76sl

To: Bob <sip:bob@biloxi.example.com>; tag=3flal12sf

Call-ID: 3848276298220188511 latlanta.example.com

CSeq: 1 INVITE

Proxy-Authenticate: Digest realm="atlanta.example.com", qop="auth", nonce="f84f1cec4le6cbe5aea9c8e88d359",

opaque="", stale=FALSE, algorithm=MD5

Content-Length: 0 
F3 ACK Alice $\rightarrow$ Proxy 1

ACK sip:bob@biloxi.example.com SIP/2.0

Via: SIP/2.0/TCP client.atlanta. example.com:5060; branch=z9hG4bK74b43

Max-Forwards: 70

From: Alice <sip:alicelatlanta.example.com>; tag=9fxced76sl

To: Bob <sip:bob@biloxi.example.com>; tag=3flal12sf

Call-ID: 3848276298220188511 eatlanta.example.com

CSeq: 1 ACK

Content-Length: 0

/* Alice responds be re-sending the INVITE with authentication credentials in it. */

F4 INVITE Alice $->$ Proxy 1

INVITE sip:bob@biloxi.example.com SIP/2.0

Via: SIP/2.0/TCP client.atlanta.example.com:5060; branch=z9hG4bK74bf 9 Max-Forwards: 70

Route: <sip:ssl.atlanta.example.com; lr>

From: Alice <sip:alicelatlanta.example.com>; tag=9fxced76sl

To: Bob <sip:bobebiloxi.example.com>

Call-ID: 3848276298220188511 @atlanta.example.com

CSeq: 2 INVITE

Contact: <sip:alicedclient.atlanta.example.com; transport=tcp>

Proxy-Authorization: Digest username="alice",

realm="atlanta.example.com",

nonce="wf84f1ceczx41ae6cbe5aea9c8e88d359", opaque=" ", uri="sip:bob@biloxi.example.com", response $=" 42 \mathrm{ce} 3 \mathrm{cef} 44 \mathrm{~b} 22 \mathrm{f50c6a6071bc8"}$

Content-Type: application/sdp

Content-Length: 151

$\mathrm{V}=0$

o=alice 28908445262890844526 IN IP4 client.atlanta.example.com

$\mathrm{S}=-$

$\mathrm{C}=\mathrm{IN}$ IP 4 192.0.2.101

$t=0 \quad 0$

m=audio 49172 RTP/AVP 0

a =rtpmap: 0 PCMU $/ 8000$

/* Proxy 1 accepts the credentials and forwards the INVITE to Proxy 2. Client for Alice prepares to receive data on port 49172 from the network. */ 


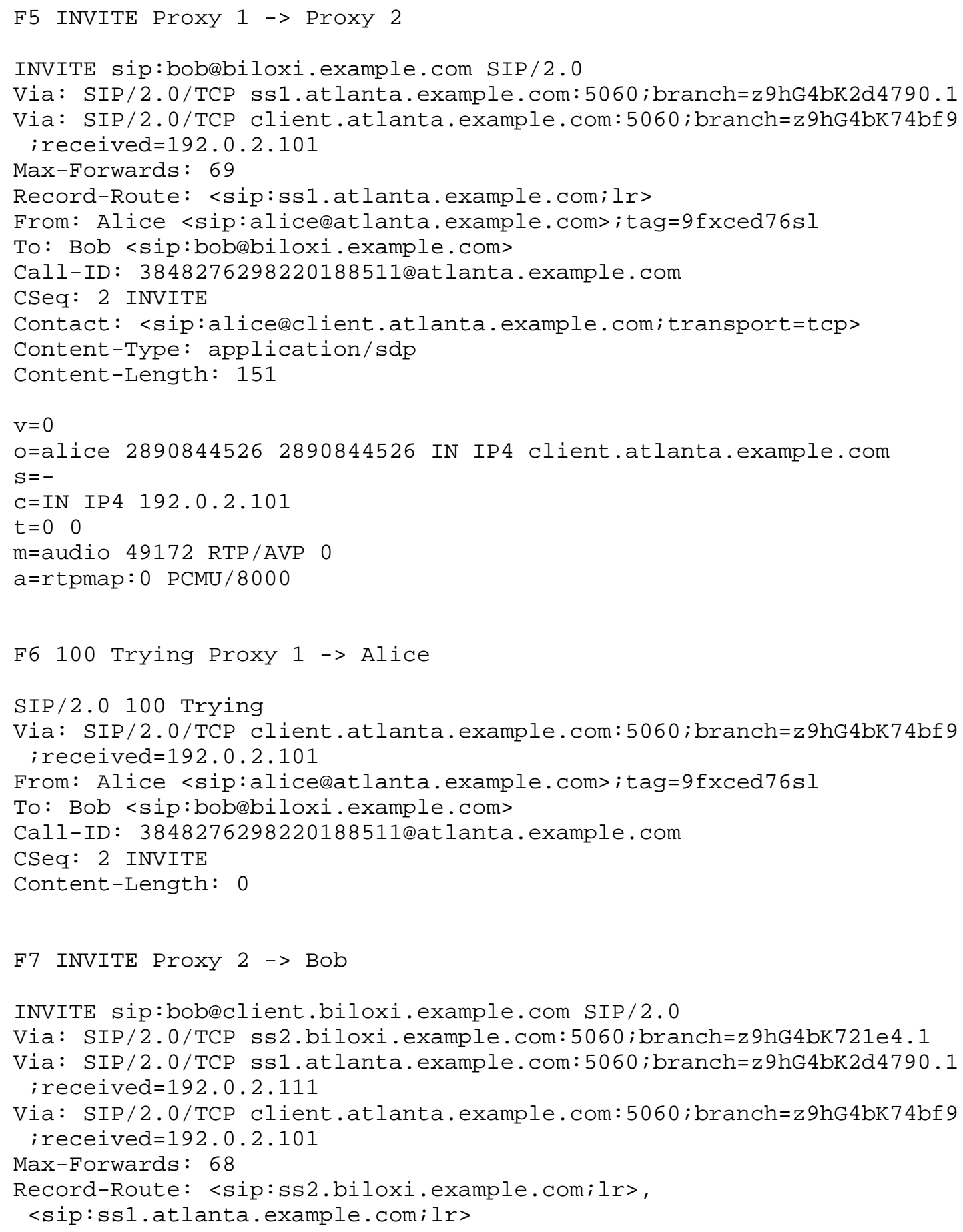




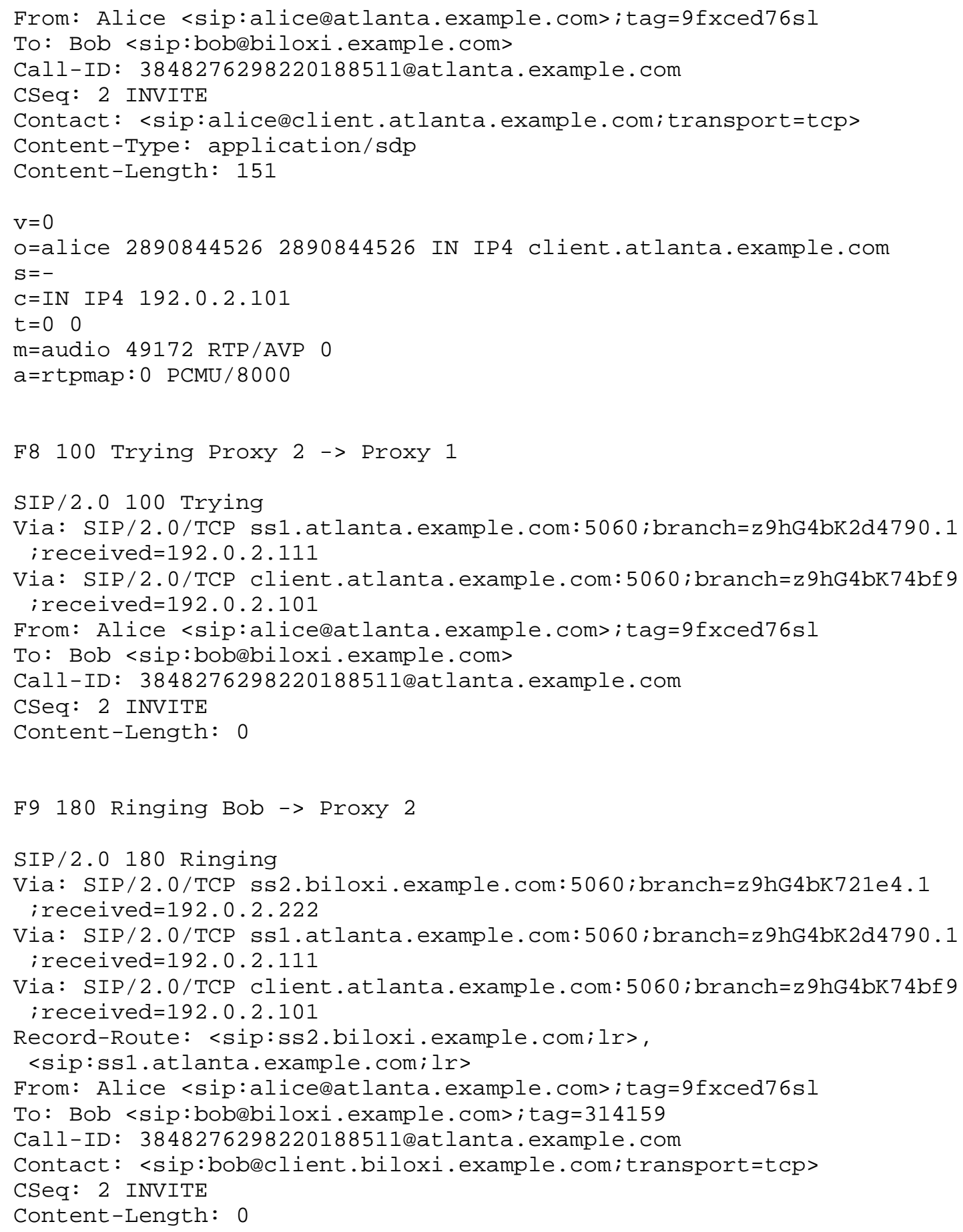


F10 180 Ringing Proxy $2 \rightarrow$ Proxy 1

SIP/2.0 180 Ringing

Via: SIP/2.0/TCP ss1.atlanta. example.com:5060; branch=z9hG4bK2d4790.1 ; received $=192.0 .2 .111$

Via: SIP/2.0/TCP client.atlanta.example.com:5060; branch=z9hG4bK74bf 9 ; received=192.0.2.101

Record-Route: <sip:ss2.biloxi.example.com;lr>, <sip:ssl.atlanta.example.com;lr>

From: Alice <sip:alicelatlanta.example.com>; tag=9fxced76sl

To: Bob <sip:bob@biloxi.example.com>; tag=314159

Call-ID: 3848276298220188511 @atlanta.example.com

Contact: <sip:bob@client.biloxi.example.com; transport=tcp>

CSeq: 2 INVITE

Content-Length: 0

F11 180 Ringing Proxy 1 -> Alice

SIP/2.0 180 Ringing

Via: SIP/2.0/TCP client.atlanta. example.com:5060; branch=z9hG4bK74bf 9 ; received=192.0.2.101

Record-Route: <sip:ss2.biloxi.example.com;lr>, <sip:ss1.atlanta.example.com; lr>

From: Alice <sip:alicelatlanta.example.com>; tag=9fxced76sl

To: Bob <sip:bobebiloxi.example.com>; tag=314159

Call-ID: 3848276298220188511 eatlanta.example.com

Contact: <sip:bobdclient.biloxi.example.com; transport=tcp>

CSeq: 2 INVITE

Content-Length: 0

F12 200 OK Bob $->$ Proxy 2

SIP $/ 2.0200$ OK

Via: SIP/2.0/TCP ss2.biloxi.example.com:5060; branch=z9hG4bK721e4.1 ; received $=192.0 .2 .222$

Via: SIP/2.0/TCP ss1.atlanta.example.com:5060; branch=z 9hG4bK2d4790.1 ; received=192.0.2.111

Via: SIP/2.0/TCP client.atlanta. example.com:5060; branch=z9hG4bK74bf 9 i received=192.0.2.101

Record-Route: <sip:ss2.biloxi.example.com; lr>, <sip:ssl.atlanta.example.com;lr>

From: Alice <sip:alicelatlanta. example.com>; tag=9fxced76sl

To: Bob <sip:bob@biloxi.example.com>; tag=314159

Call-ID: 3848276298220188511 datlanta.example.com

CSeq: 2 INVITE 


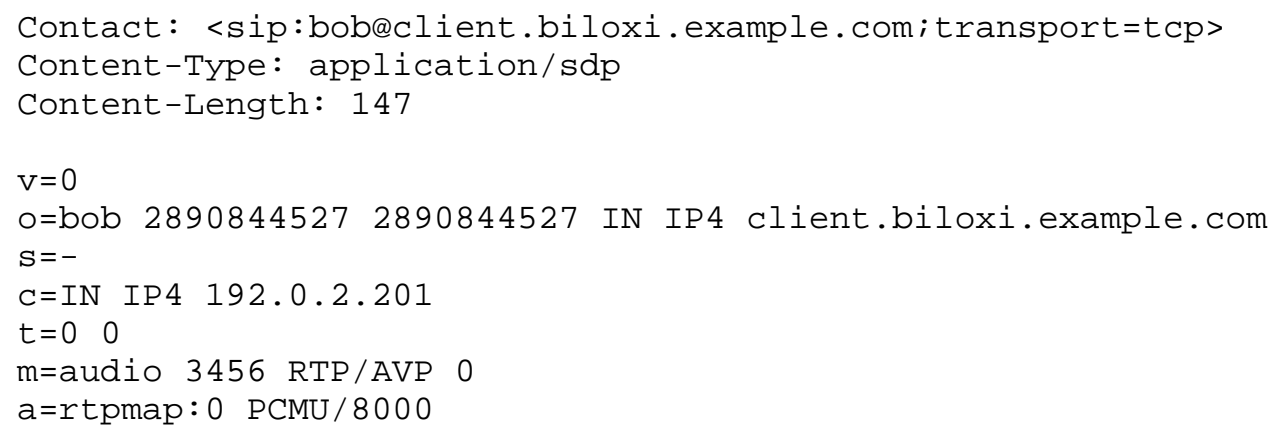




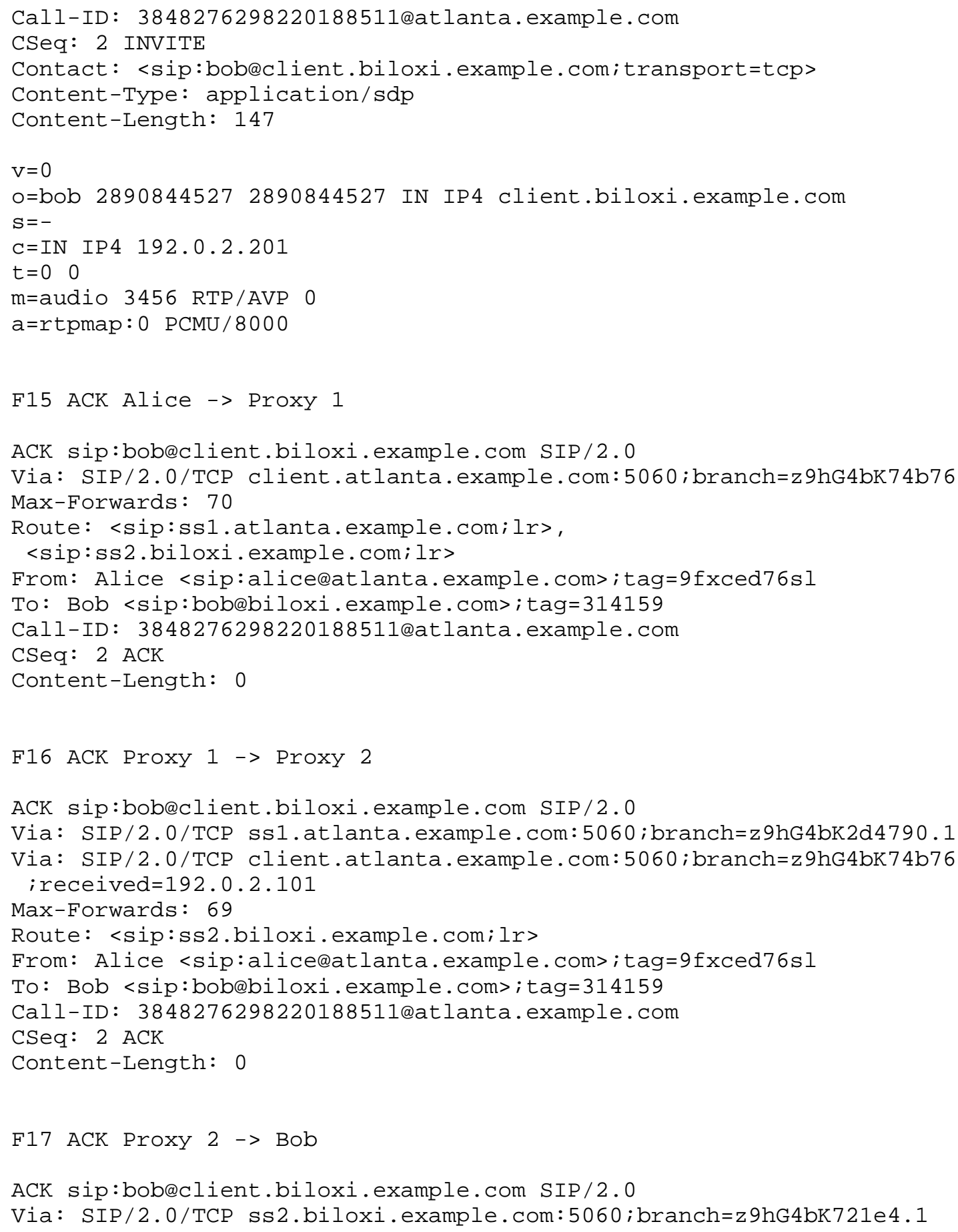


Via: SIP/2.0/TCP ss1.atlanta. example.com:5060; branch=z9hG4bK2d4790.1 ; received $=192.0 .2 .111$

Via: SIP/2.0/TCP client.atlanta.example.com:5060;branch=z9hG4bK74b76 ; received=192.0.2.101

Max-Forwards: 68

From: Alice <sip:alicelatlanta.example.com>; tag=9fxced76sl

To: Bob <sip:bobebiloxi.example.com>; tag=314159

Call-ID: 3848276298220188511 datlanta.example.com

CSeq: 2 ACK

Content-Length: 0

/* RTP streams are established between Alice and Bob */

/* Bob Hangs Up with Alice. */

/* Again, note that the CSeq is NOT 3. Alice and Bob maintain their own separate CSeq counts */

F18 BYE Bob -> Proxy 2

BYE sip:alicedclient.atlanta.example.com SIP/2.0

Via: SIP/2.0/TCP client.biloxi.example.com:5060; branch=z9hG4bKnashds 7 Max-Forwards: 70

Route: <sip:ss2.biloxi.example.com; lr>, <sip:ssl.atlanta.example.com;lr>

From: Bob <sip:bob@biloxi.example.com>; tag=314159

To: Alice <sip:alicedatlanta.example.com>; tag=9fxced76sl

Call-ID: 3848276298220188511 latlanta.example.com

CSeq: 1 BYE

Content-Length: 0

F19 BYE Proxy $2->$ Proxy 1

BYE sip:alicedclient.atlanta.example.com SIP/2.0

Via: SIP/2.0/TCP ss2.biloxi.example.com:5060; branch=z9hG4bK721e4.1

Via: SIP/2.0/TCP client.biloxi.example.com:5060; branch=z9hG4bKnashds 7 ; received=192.0.2.201

Max-Forwards: 69

Route: <sip:ssl.atlanta.example.com; lr>

From: Bob <sip:bob@biloxi.example.com>; tag=314159

To: Alice <sip:alicedatlanta.example.com>; tag=9fxced76sl

Call-ID: 3848276298220188511 eatlanta.example.com

CSeq: 1 BYE

Content-Length: 0 


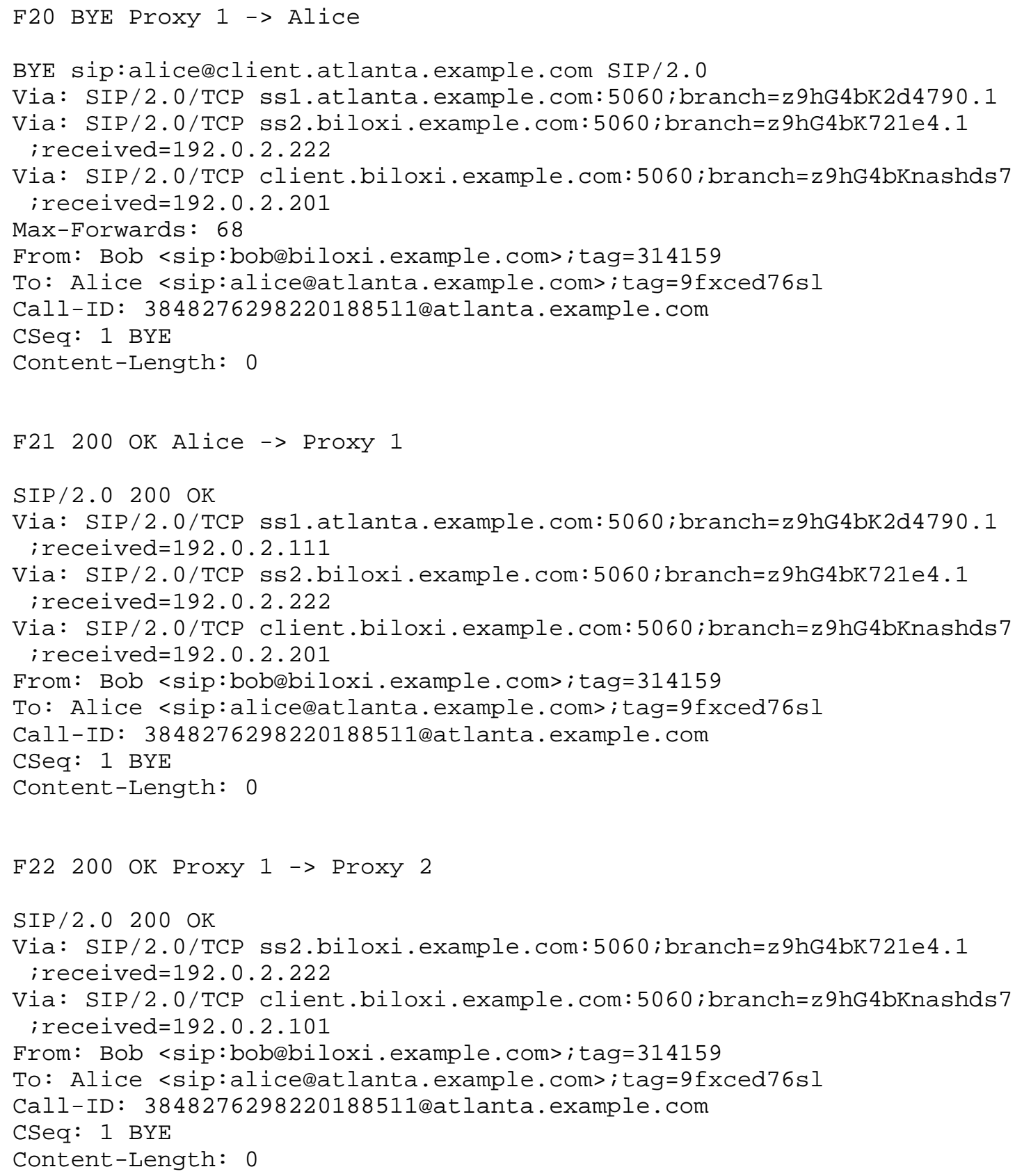


F23 200 OK Proxy $2 \rightarrow$ Bob

SIP/2.0 200 OK

Via: SIP/2.0/TCP client.biloxi.example.com:5060; branch=z9hG4bKnashds 7 ; received=192.0.2.201

From: Bob <sip:boblbiloxi.example.com>; tag=314159

To: Alice <sip:alicedatlanta.example.com>; tag=9fxced76sl

Call-ID: 3848276298220188511 datlanta.example.com

CSeq: 1 BYE

Content-Length: 0 
3.3. Session with Multiple Proxy Authentication

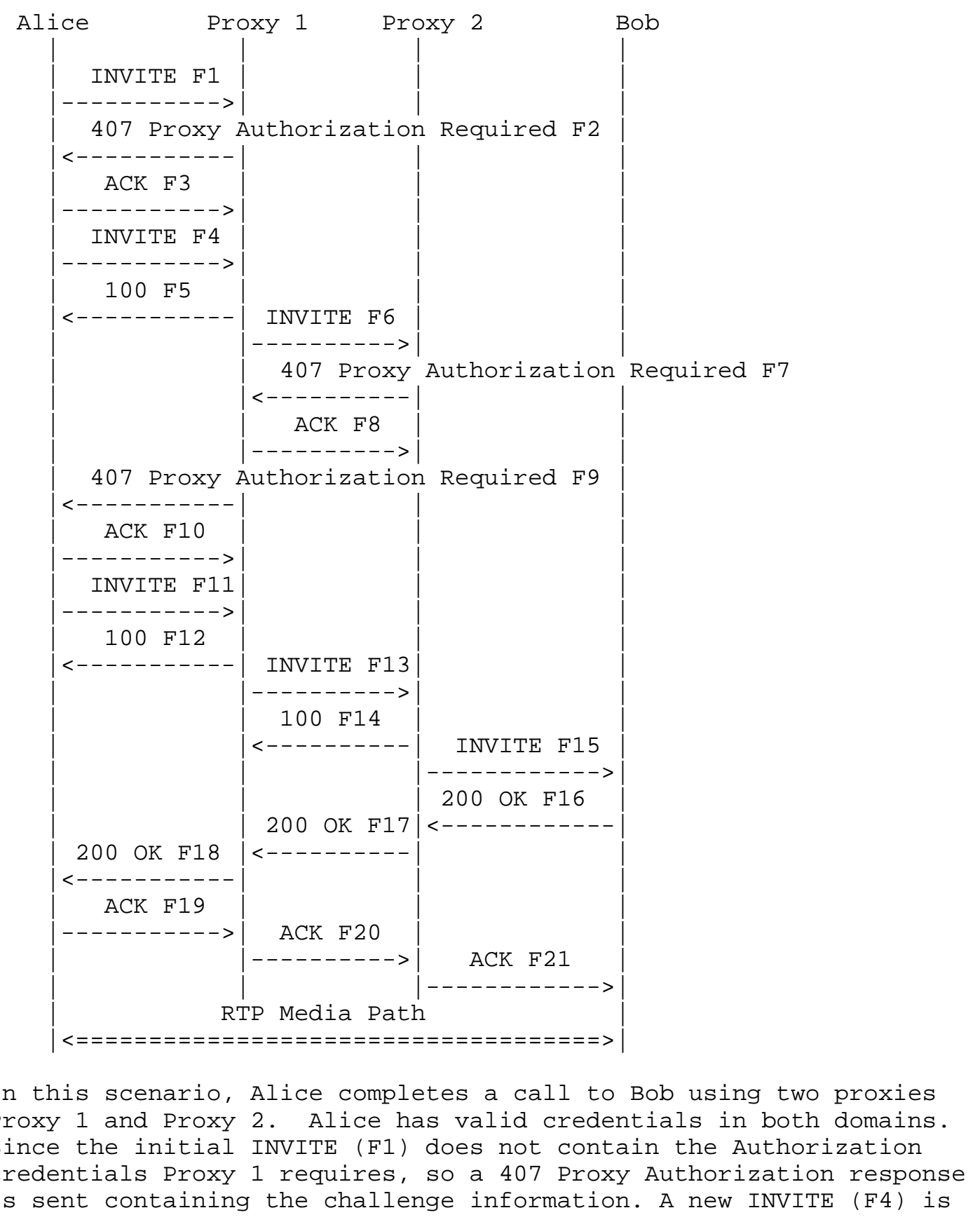




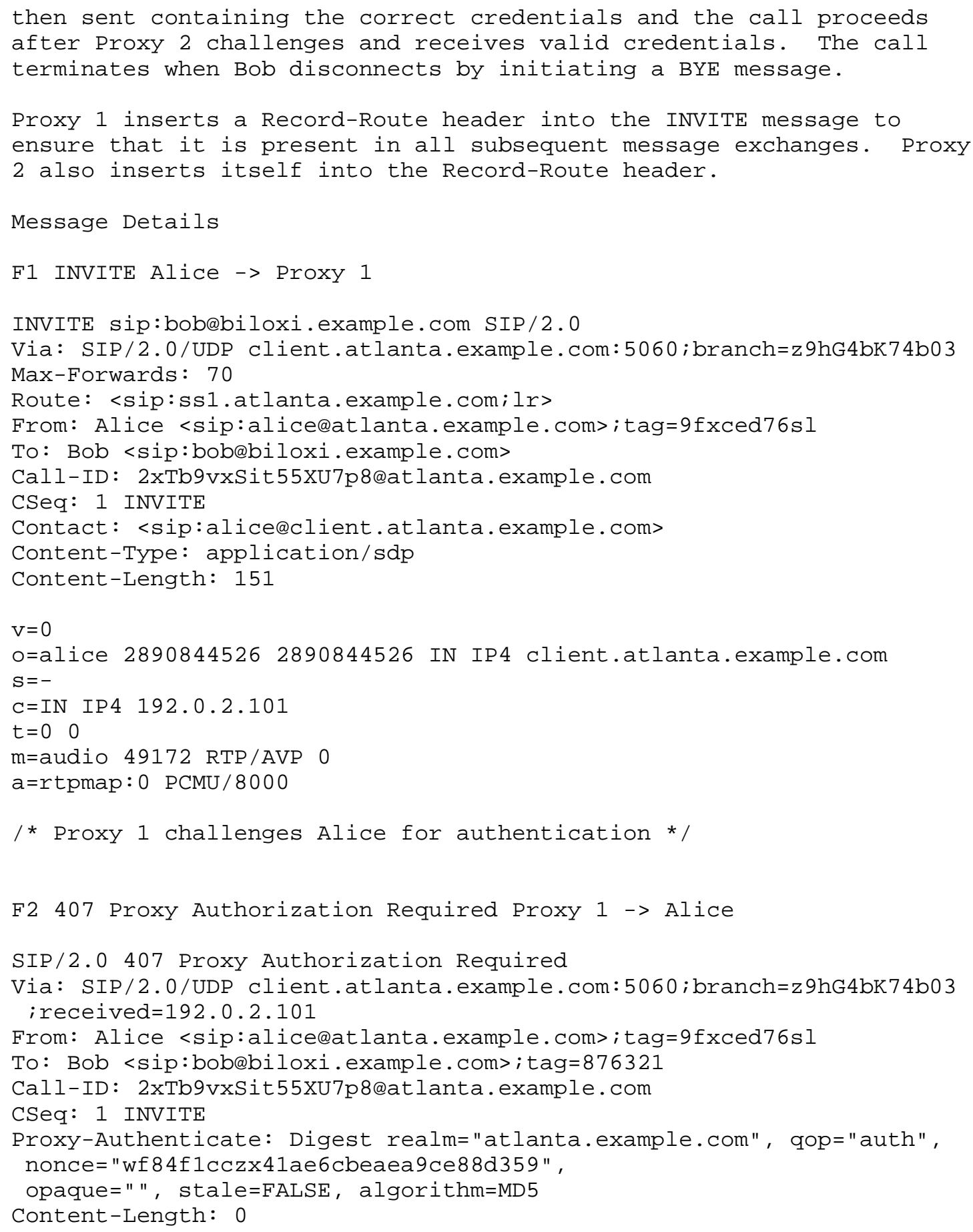




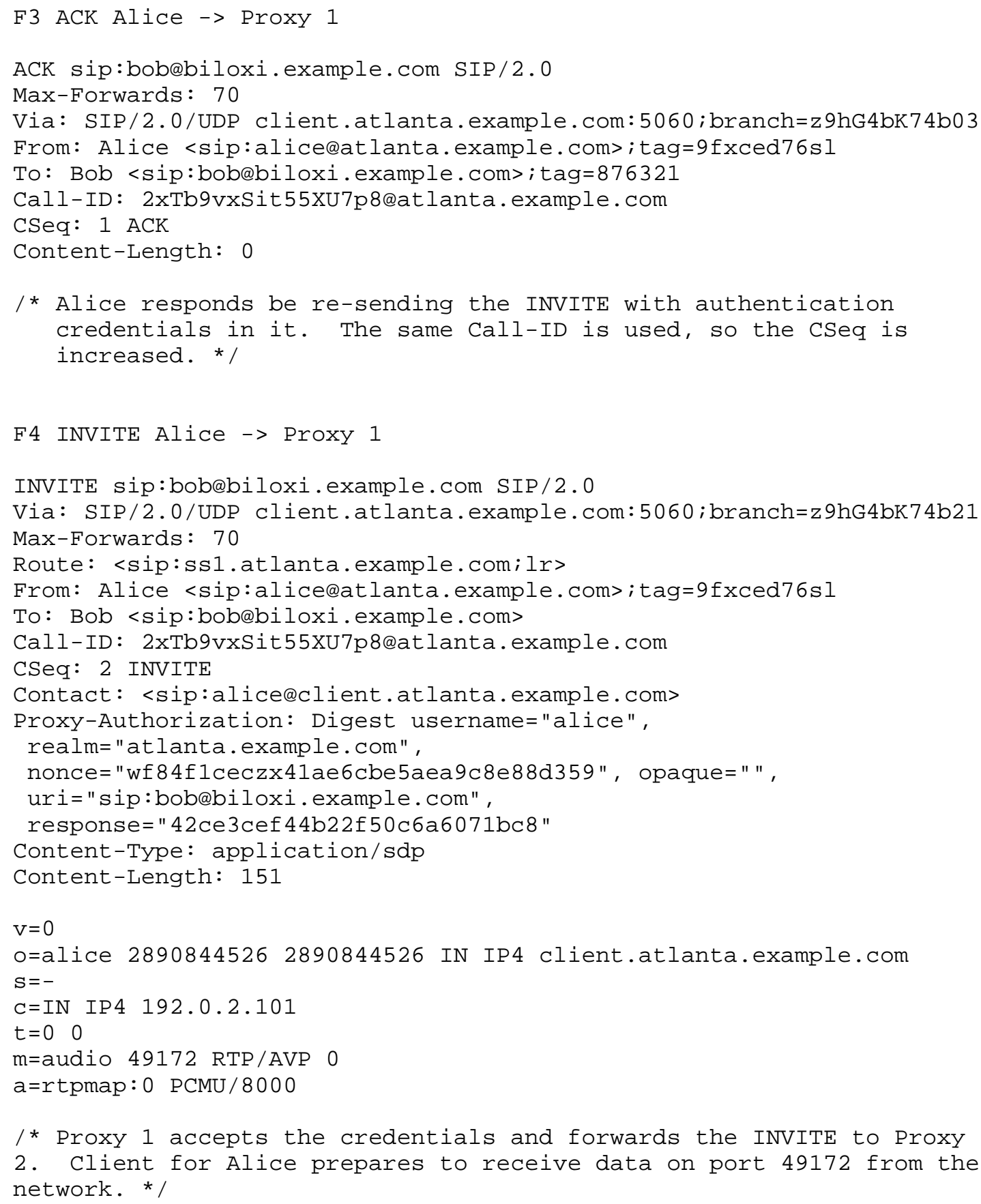




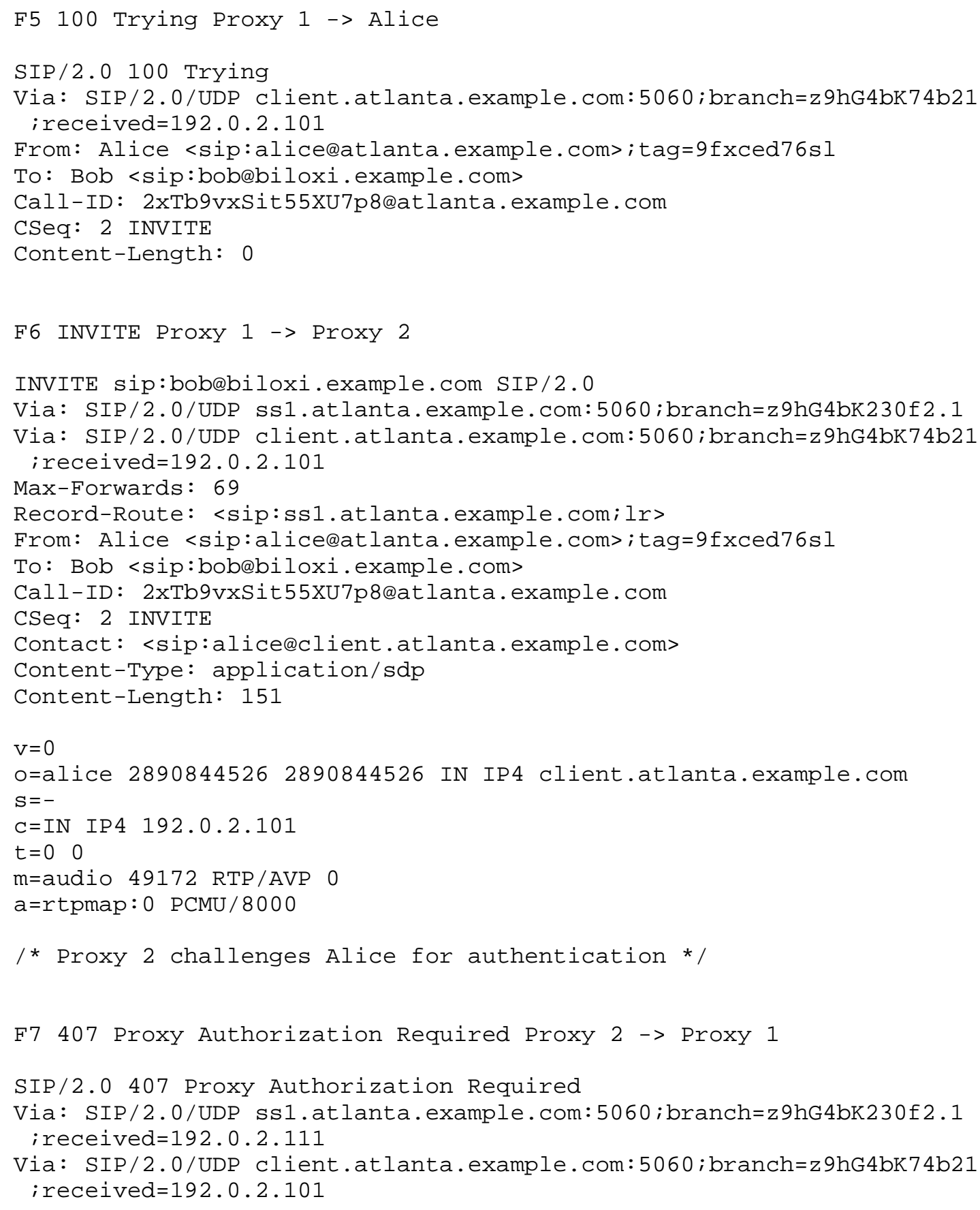




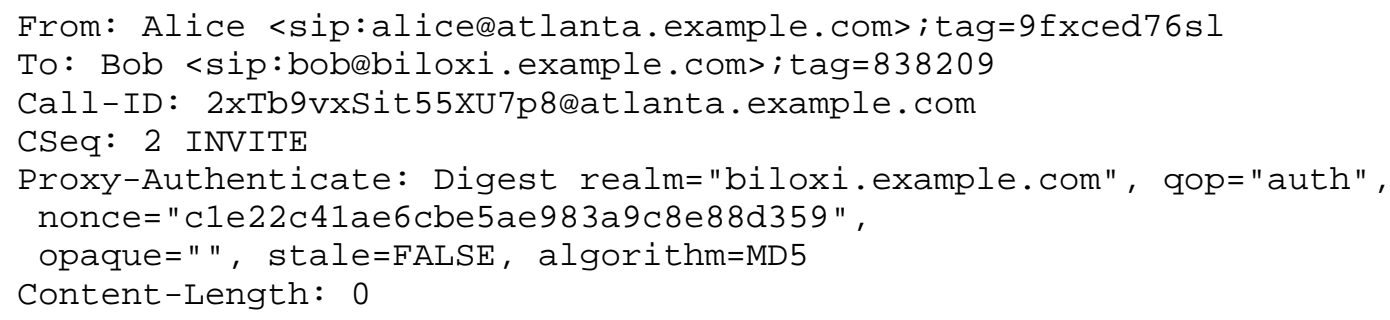




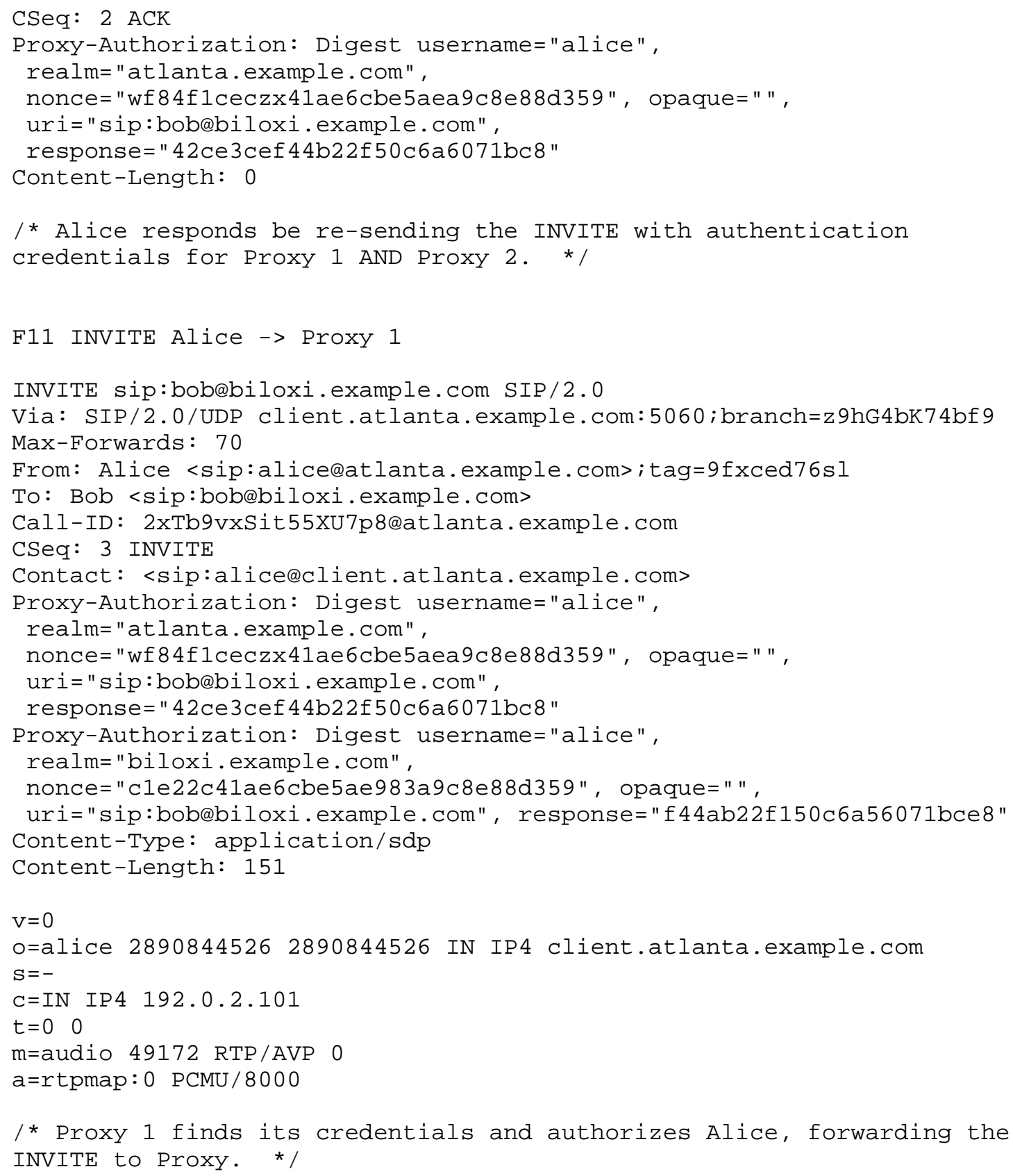




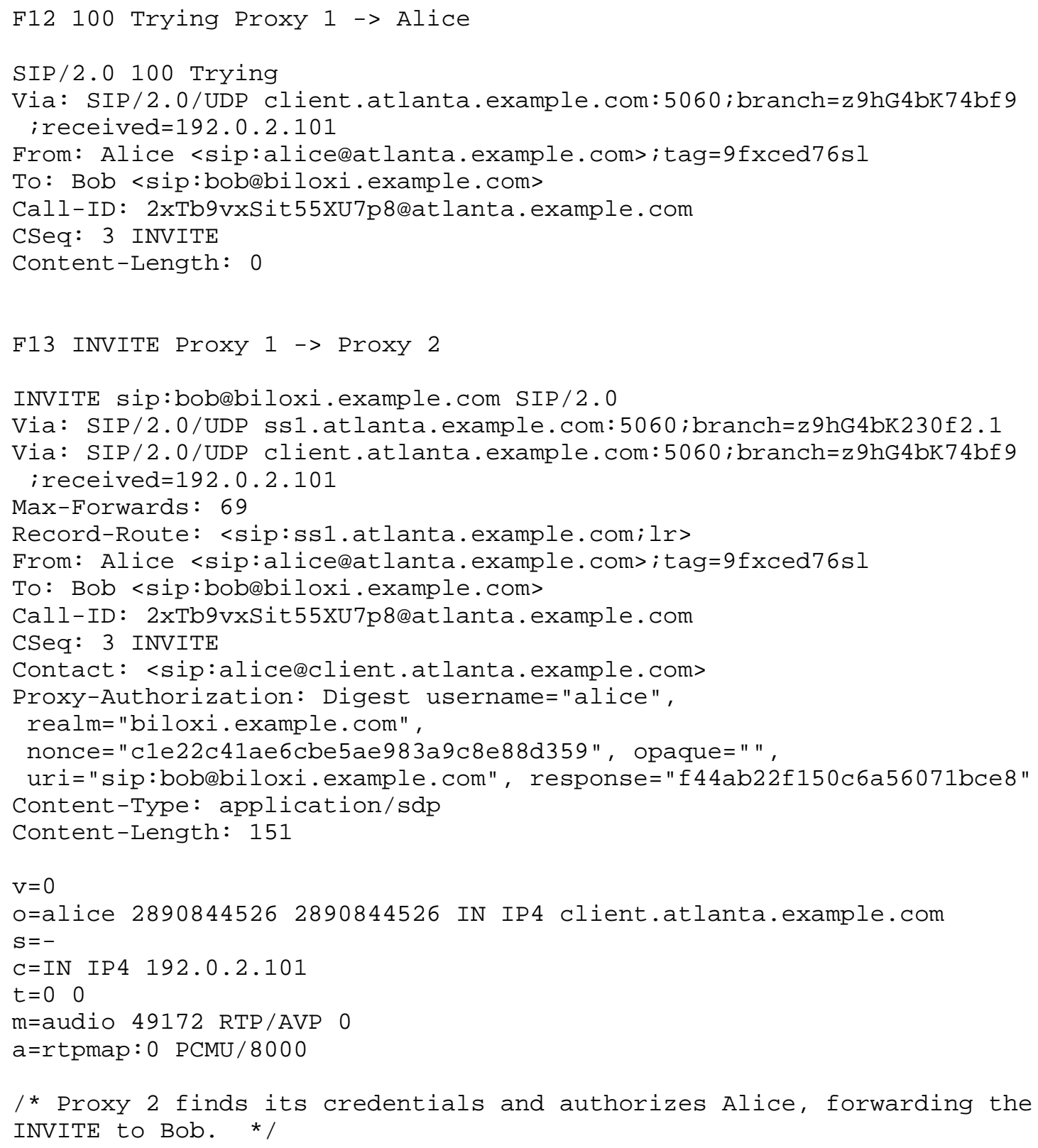




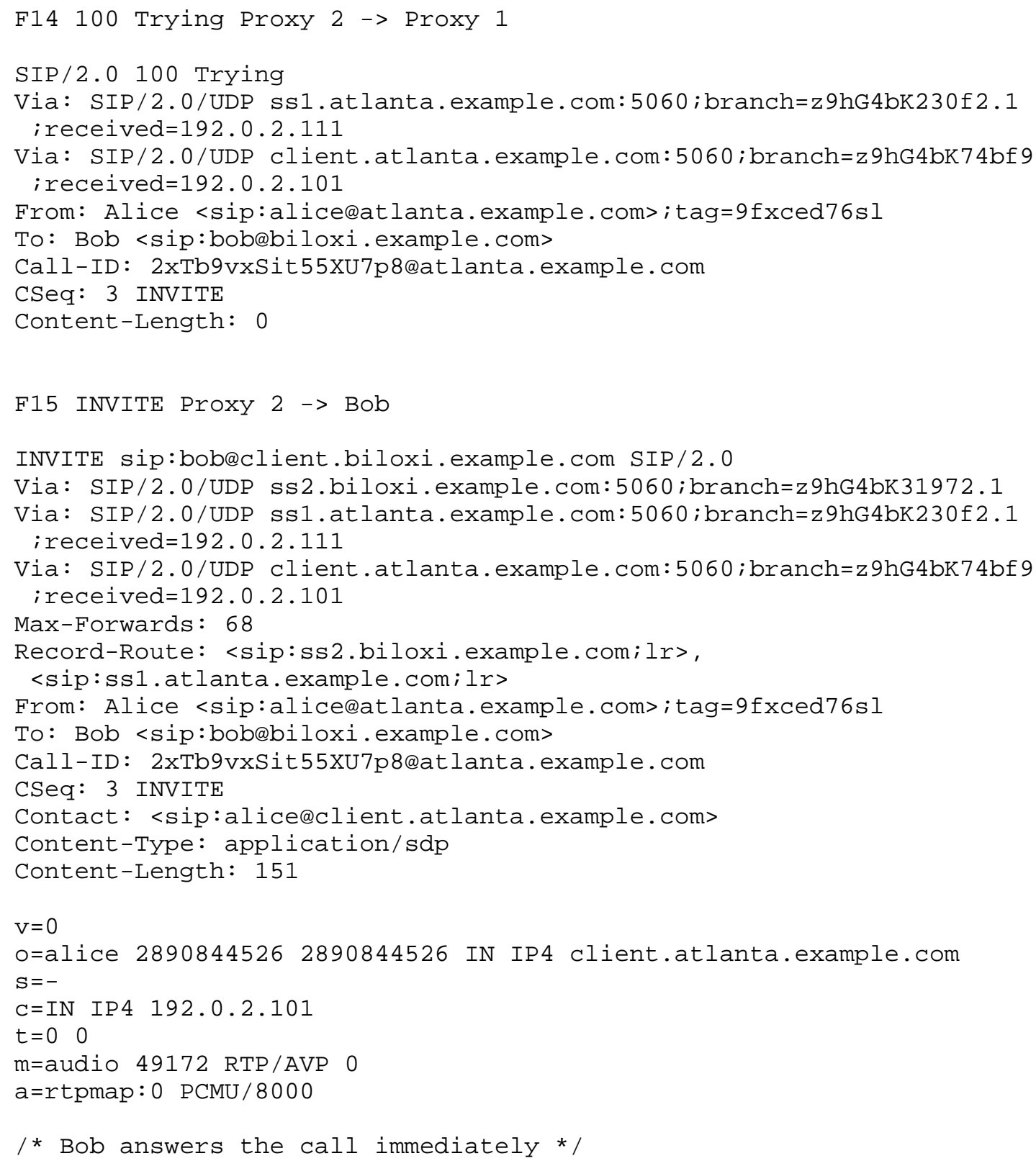




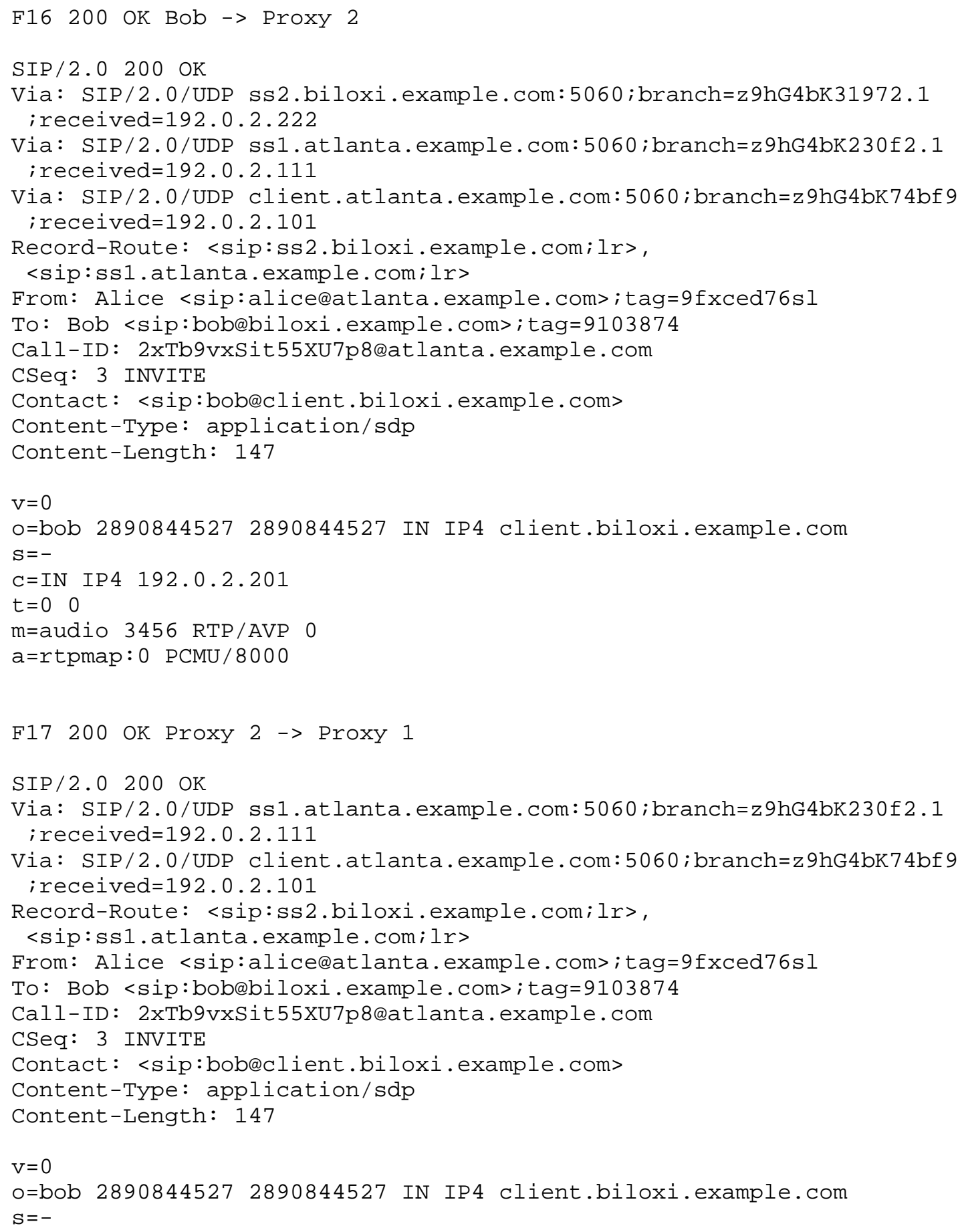




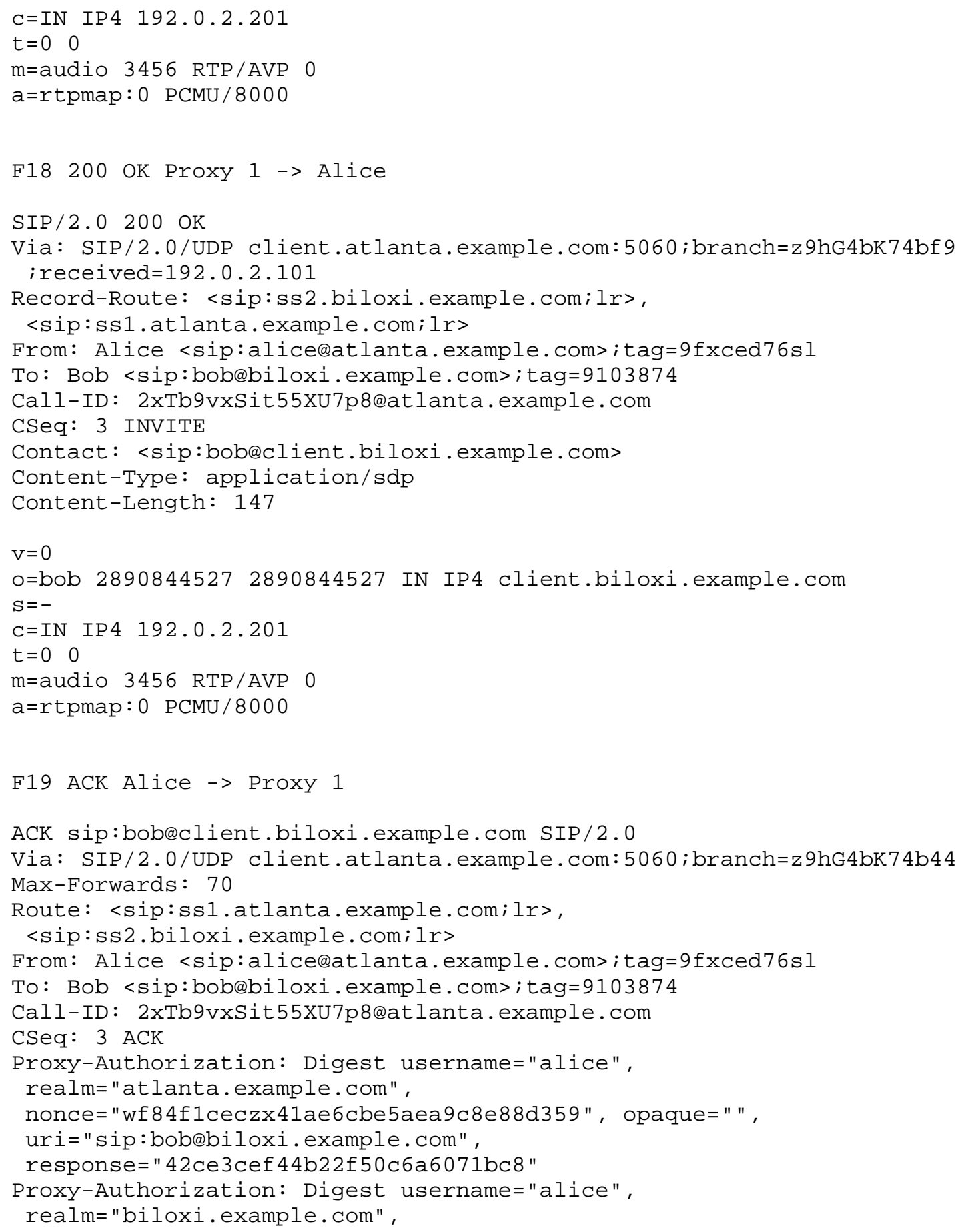




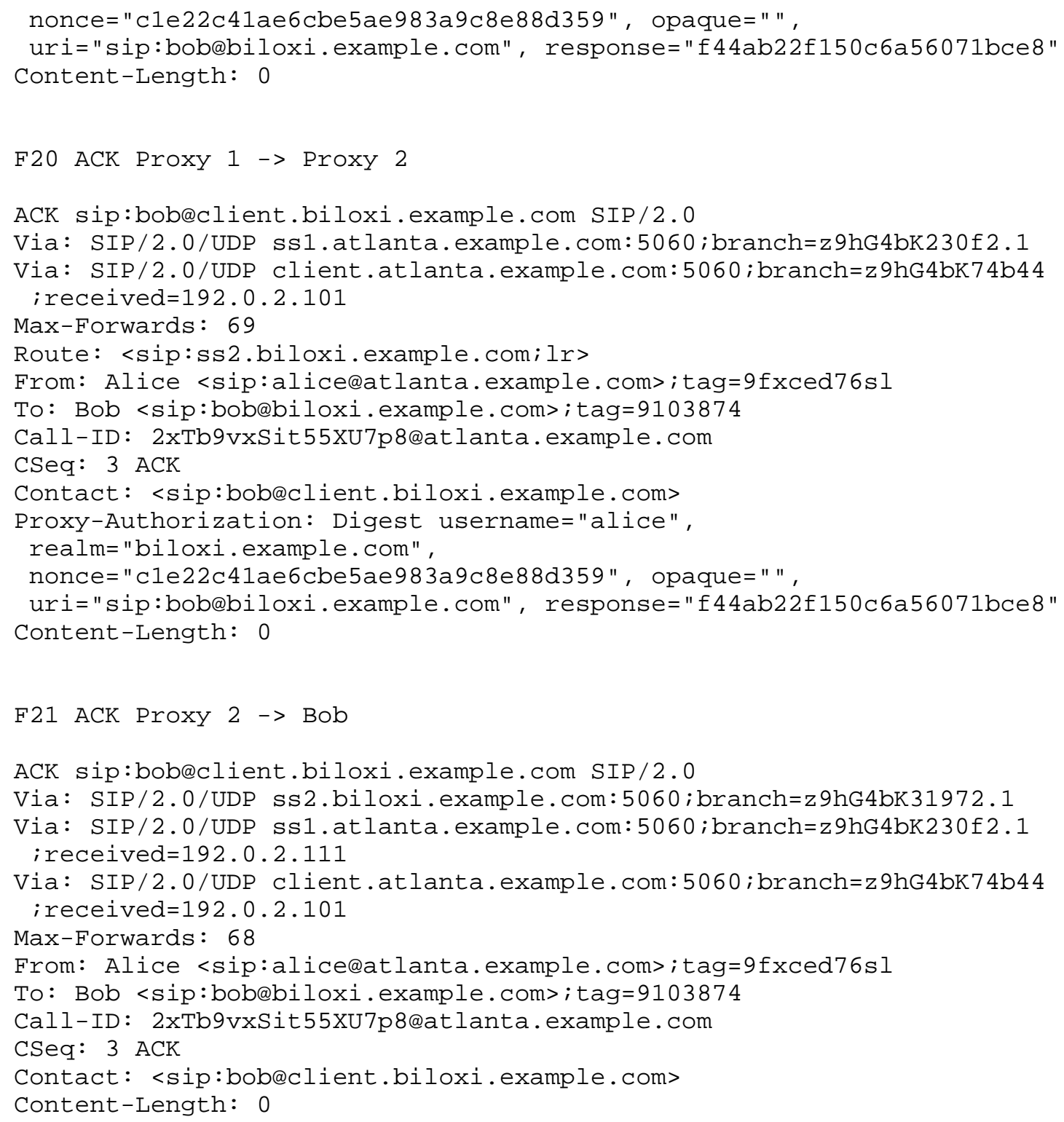


3.4. Successful Session with Proxy Failure

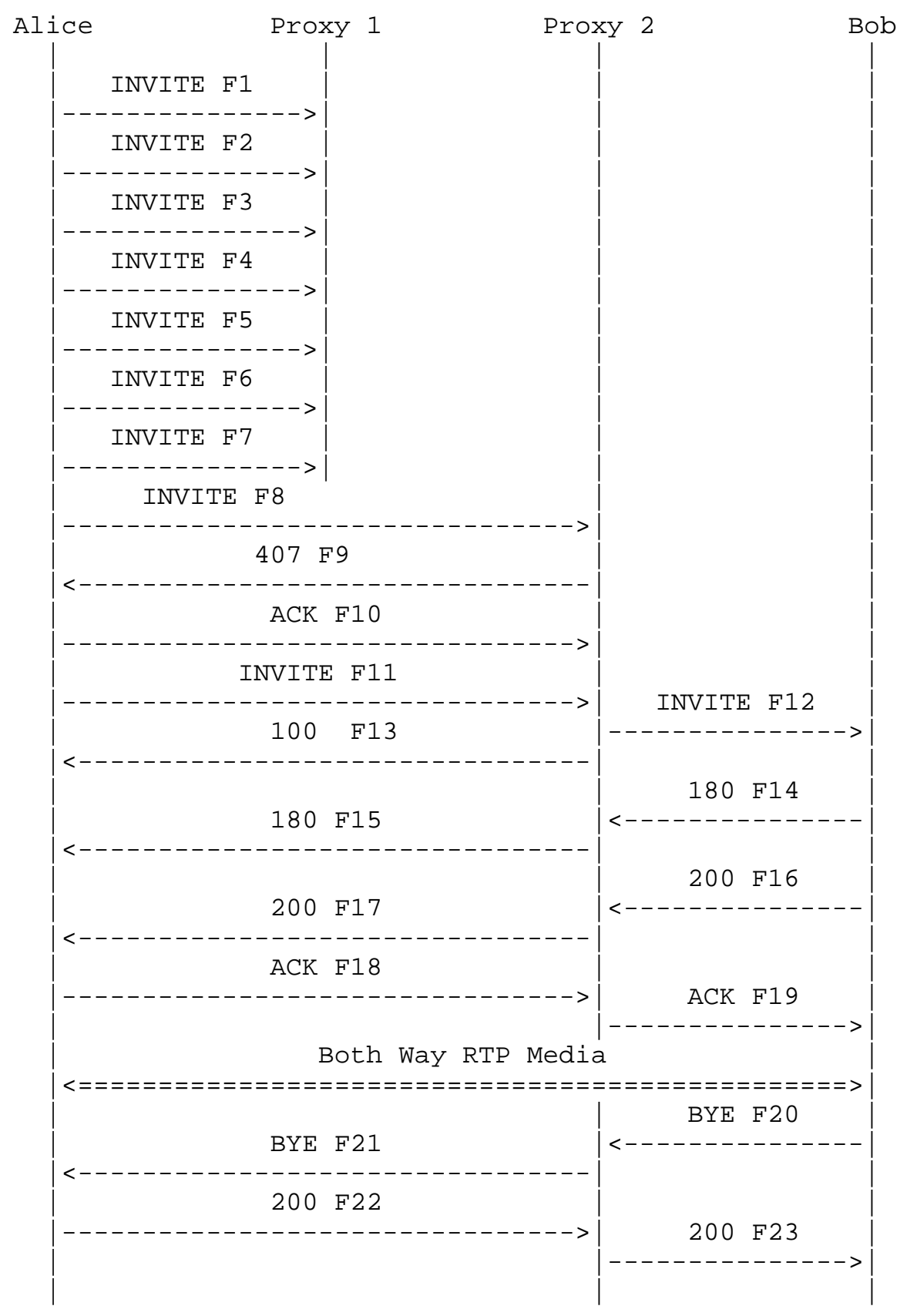




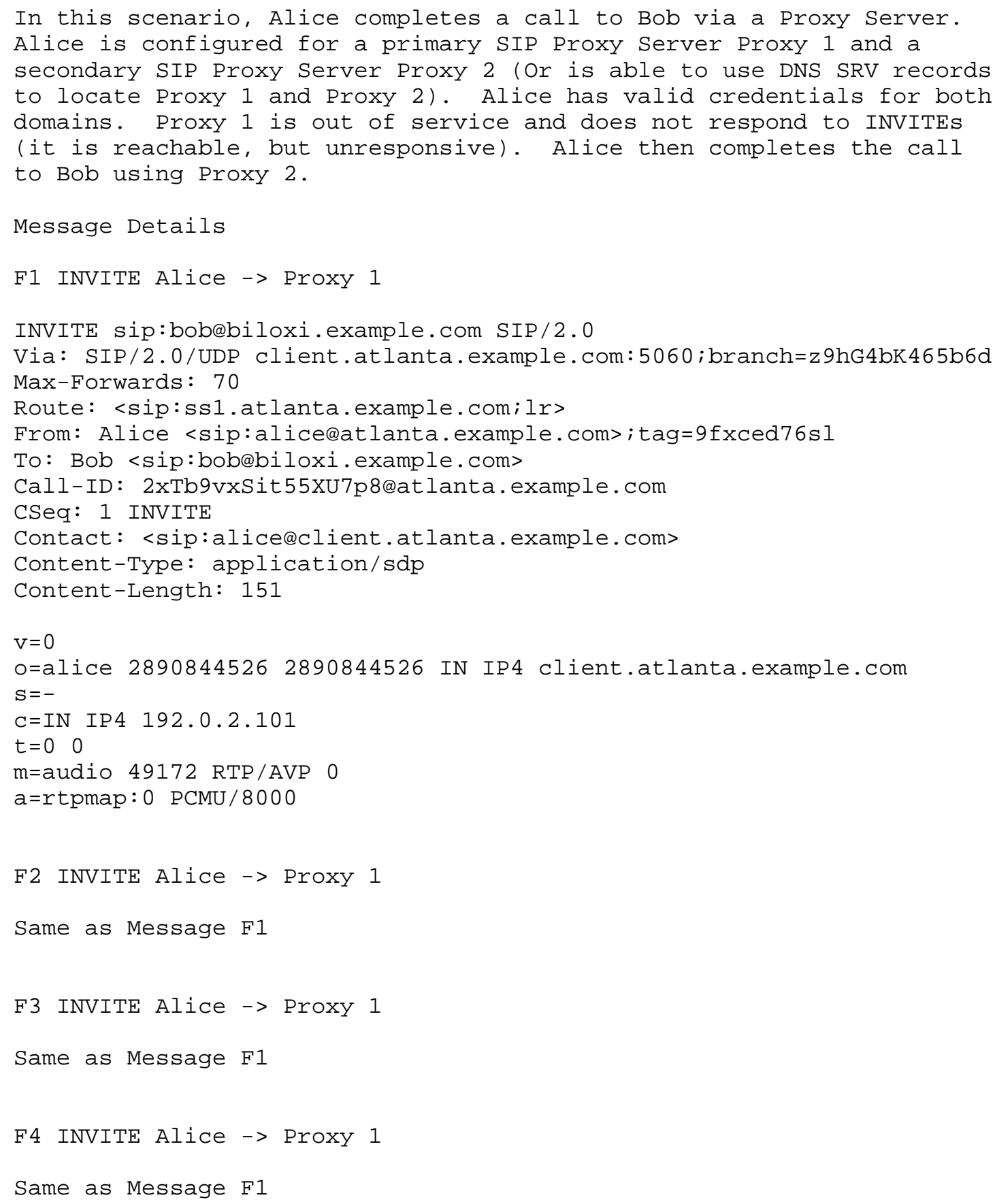




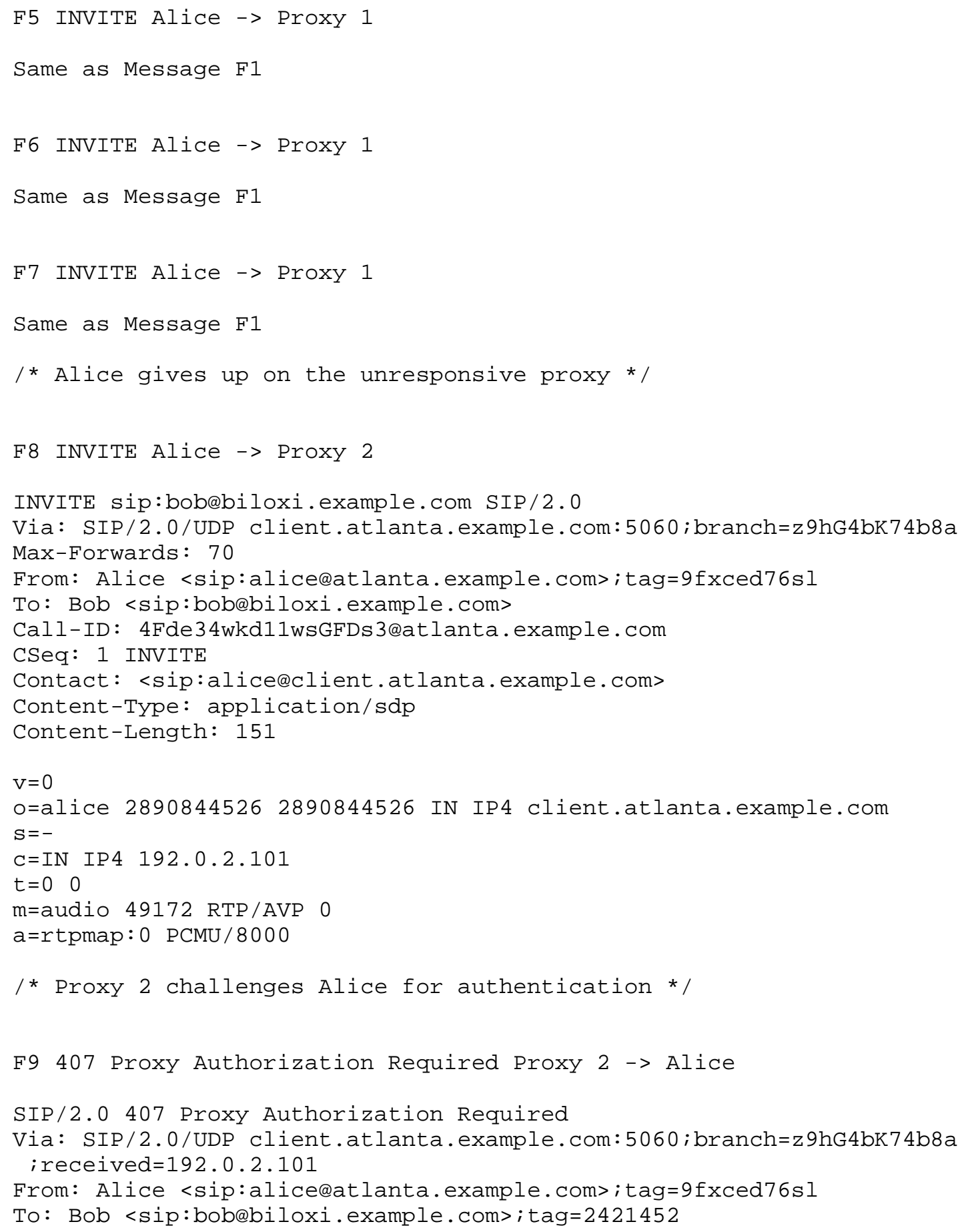




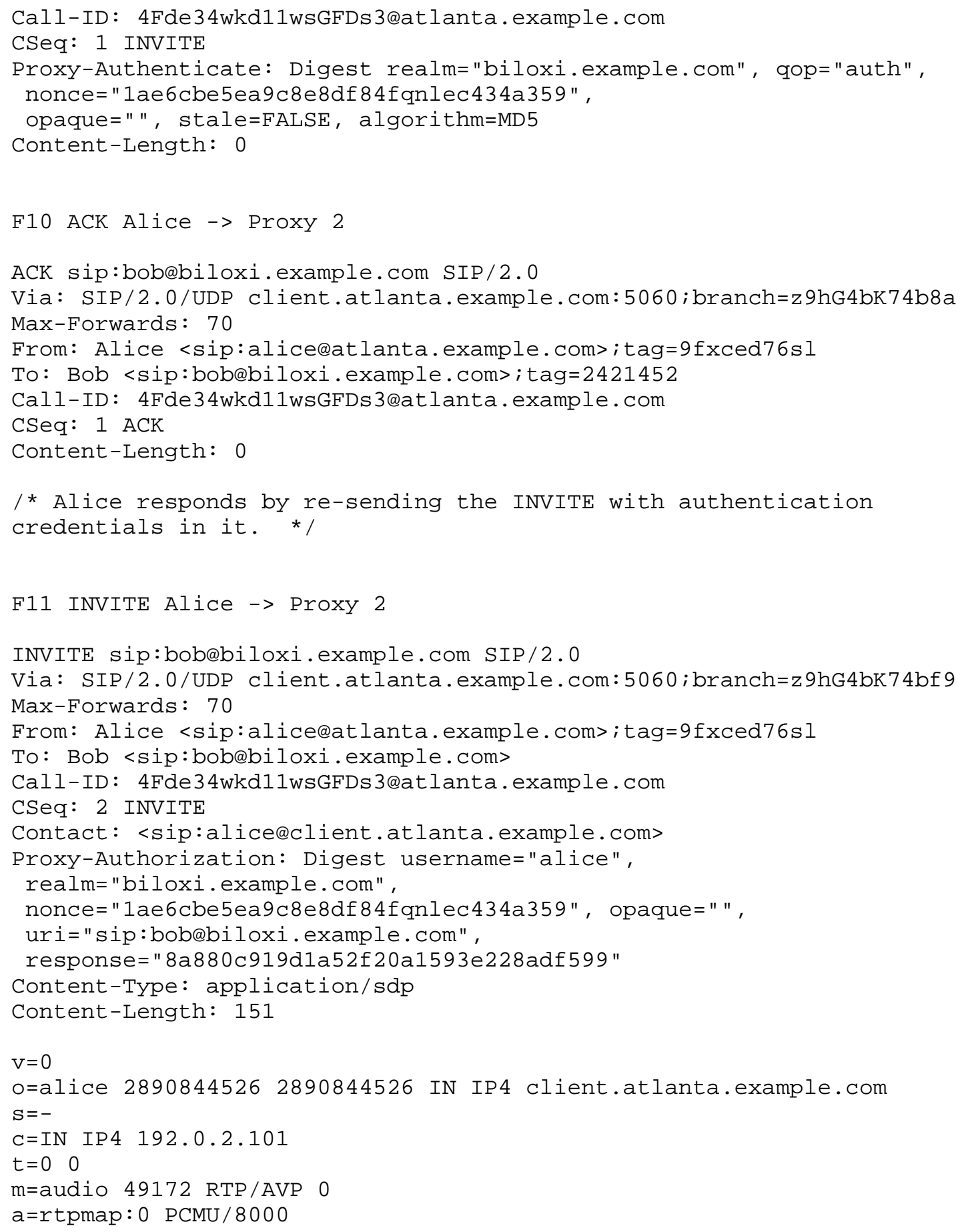


/* Proxy 2 accepts the credentials and forwards the INVITE to Bob. Client for Alice prepares to receive data on port 49172 from the network. $\star /$

F12 INVITE Proxy $2->$ Bob

INVITE sip:bob@client.biloxi.example.com SIP/2.0

Via: SIP/2.0/UDP ss2.biloxi.example.com:5060; branch=z9hG4bK721e4.1

Via: SIP/2.0/UDP client.atlanta.example.com:5060; branch=z9hG4bK74bf 9 ; received $=192.0 .2 .101$

Max-Forwards: 69

Record-Route: <sip:ss2.biloxi.example.com;lr>

From: Alice <sip:alicedatlanta.example.com>; tag=9fxced76sl

To: Bob <sip:bob@biloxi.example.com>

Call-ID: 4Fde34wkd11wsGFDs3eatlanta.example.com

CSeq: 2 INVITE

Contact: <sip:alicedclient.atlanta.example.com>

Content-Type: application/sdp

Content-Length: 151

$\mathrm{V}=0$

o=alice 28908445262890844526 IN IP4 client.atlanta.example.com

$\mathrm{S}=-$

$\mathrm{C}=\mathrm{IN}$ IP 4 192.0.2.101

$t=0 \quad 0$

m=audio 49172 RTP/AVP 0

a =rtpmap:0 PCMU / 8000

F13 100 Trying Proxy 2 -> Alice

SIP/2.0 100 Trying

Via: SIP/2.0/UDP client.atlanta.example.com:5060; branch=z9hG4bK74bf 9 ; received=192.0.2.101

From: Alice <sip:alicelatlanta. example.com>; tag=9fxced76sl

To: Bob <sip:boblbiloxi.example.com>

Call-ID: 4Fde34wkd11wsGFDs3eatlanta.example.com

CSeq: 2 INVITE

Content-Length: 0

F14 180 Ringing Bob $->$ Proxy 2

SIP/2.0 180 Ringing

Via: SIP/2.0/UDP ss2.biloxi.example.com:5060; branch=z9hG4bK721e4.1 ; received=192.0.2.222 
Via: SIP/2.0/UDP client.atlanta.example.com:5060; branch=z9hG4bK74bf 9 ; received=192.0.2.101

Record-Route: <sip:ss2.biloxi.example.com; lr>

From: Alice <sip:alicelatlanta.example.com>; tag=9fxced76sl

To: Bob <sip:bobebiloxi.example.com>; tag=314159

Call-ID: 4Fde34wkd11wsGFDs3eatlanta.example.com

CSeq: 2 INVITE

Contact: <sip:bob@client.biloxi.example.com>

Content-Length: 0

F15 180 Ringing Proxy $2 \rightarrow$ Alice

SIP/2.0 180 Ringing

Via: SIP/2.0/UDP client.atlanta.example.com:5060; branch=z9hG4bK74bf 9 ; received=192.0.2.101

Record-Route: <sip:ss2.biloxi.example.com; lr>

From: Alice <sip:alicelatlanta.example.com>; tag=9fxced76sl

To: Bob <sip:bob@biloxi.example.com>; tag=314159

Call-ID: 4Fde34wkd11wsGFDs3eatlanta.example.com

CSeq: 2 INVITE

Contact: <sip:bob@client.biloxi.example.com>

Content-Length: 0

F16 200 OK Bob -> Proxy 2

SIP $/ 2.0200$ OK

Via: SIP/2.0/UDP ss2.biloxi.example.com:5060; branch=z9hG4bK721e4.1

; received=192.0.2.222

Via: SIP/2.0/UDP client.atlanta.example.com:5060; branch=z9hG4bK74bf 9

; received=192.0.2.101

Record-Route: <sip:ss2.biloxi.example.com; lr>

From: Alice <sip:alicelatlanta.example.com>; tag=9fxced76sl

To: Bob <sip:bobebiloxi.example. com>; tag=314159

Call-ID: 4Fde34wkd11wsGFDs3eatlanta.example.com

CSeq: 2 INVITE

Contact: <sip:bobdclient.biloxi.example.com>

Content-Type: application/sdp

Content-Length: 147

$\mathrm{V}=0$

o=bob 28908445272890844527 IN IP4 client.biloxi.example.com

$\mathrm{S}=-$

$\mathrm{C}=\mathrm{IN}$ IP 4 192.0.2.201

$t=0 \quad 0$

m=audio 3456 RTP/AVP 0

$\mathrm{a}=r t$ pmap : $0 \mathrm{PCMU} / 8000$ 


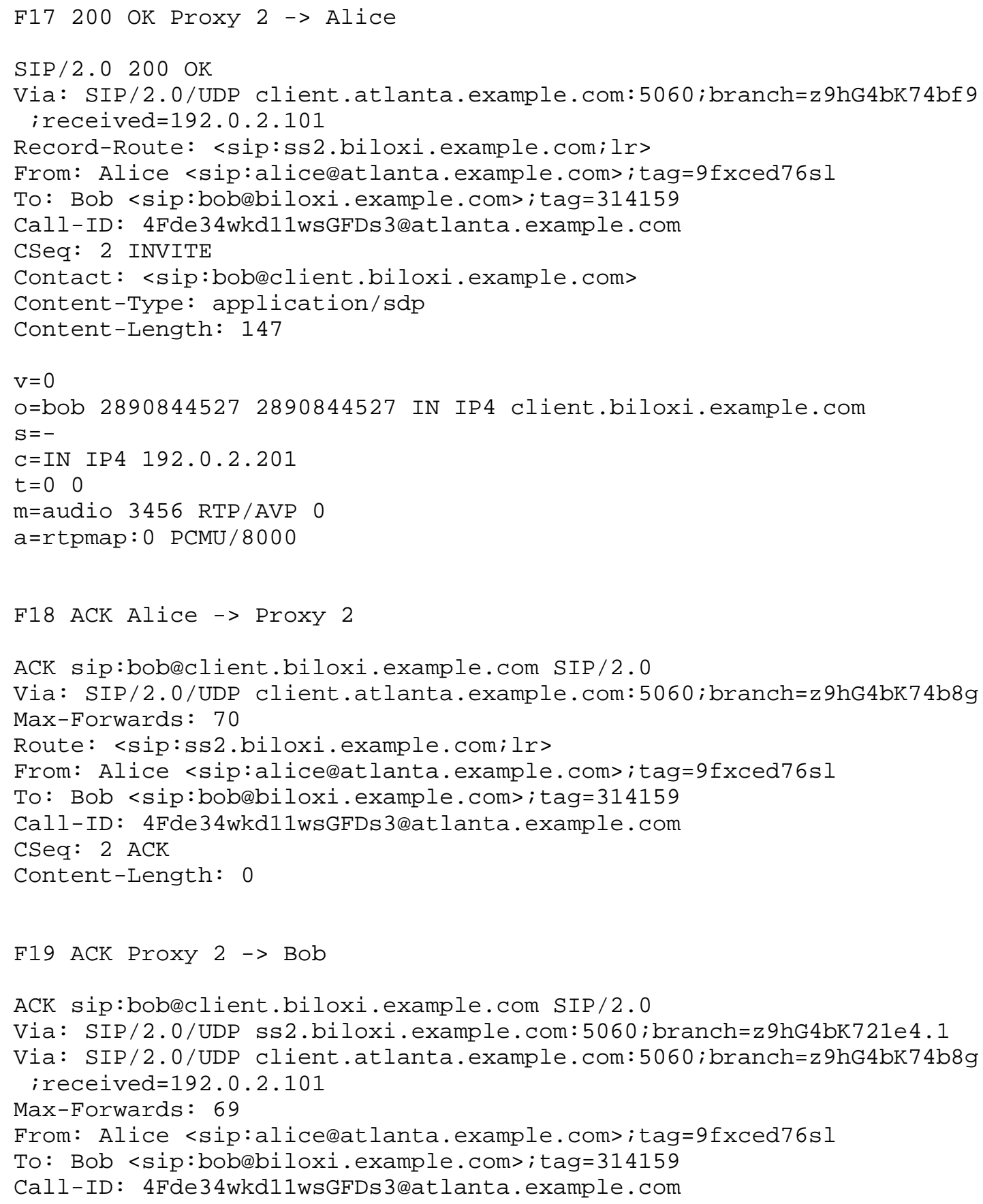




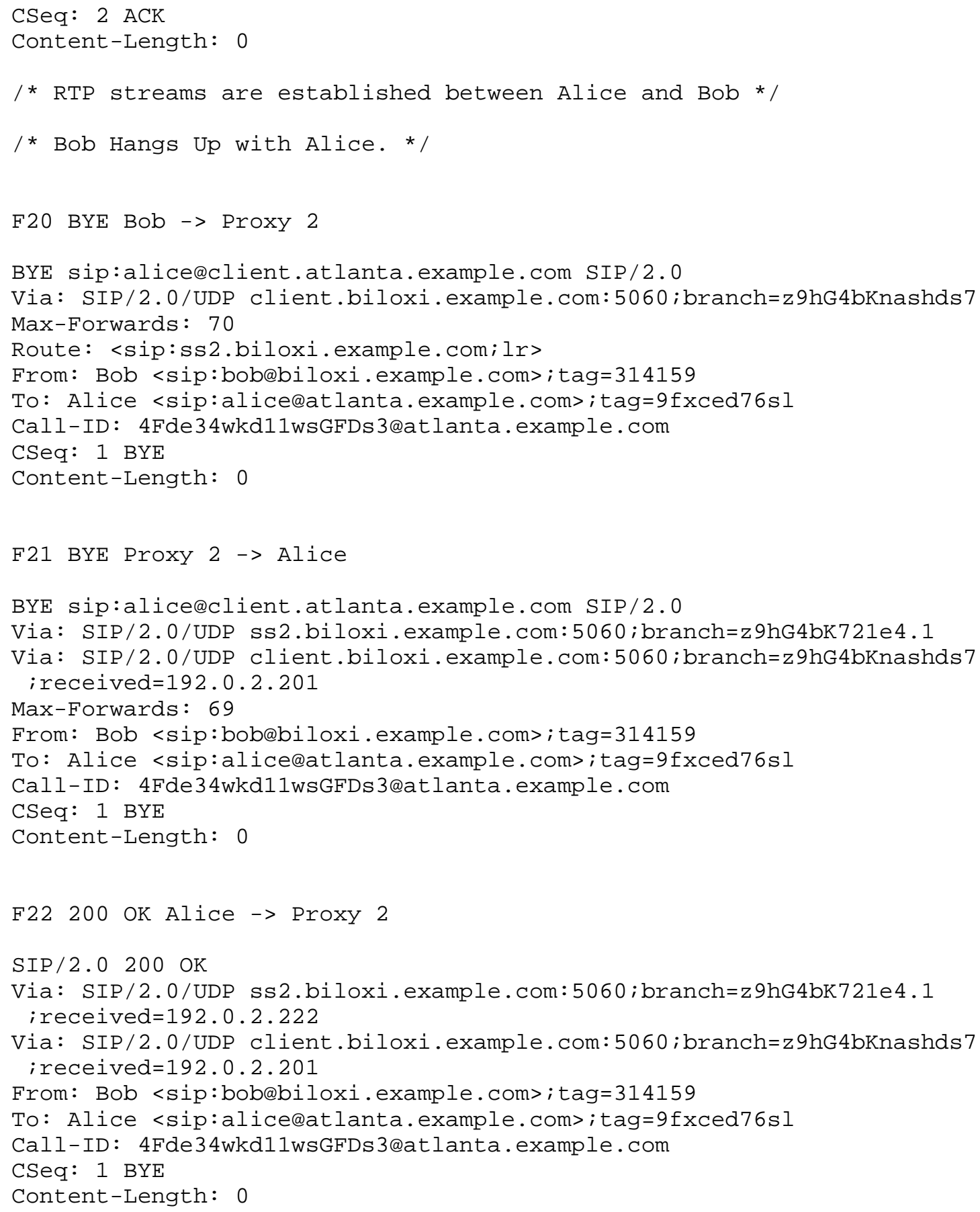


F23 200 OK Proxy $2->$ Bob

SIP $/ 2.0200$ OK

Via: SIP/2.0/UDP client.biloxi.example.com:5060; branch=z9hG4bKnashds 7 ; received $=192.0 .2 .201$

From: Bob <sip:bob@biloxi.example.com>; tag=314159

To: Alice <sip:alicelatlanta.example.com>; tag=9fxced76sl

Call-ID: 4Fde34wkd11wsGFDs3eatlanta.example.com

CSeq: 1 BYE

Content-Length: 0 
3.5. Session Through a SIP ALG

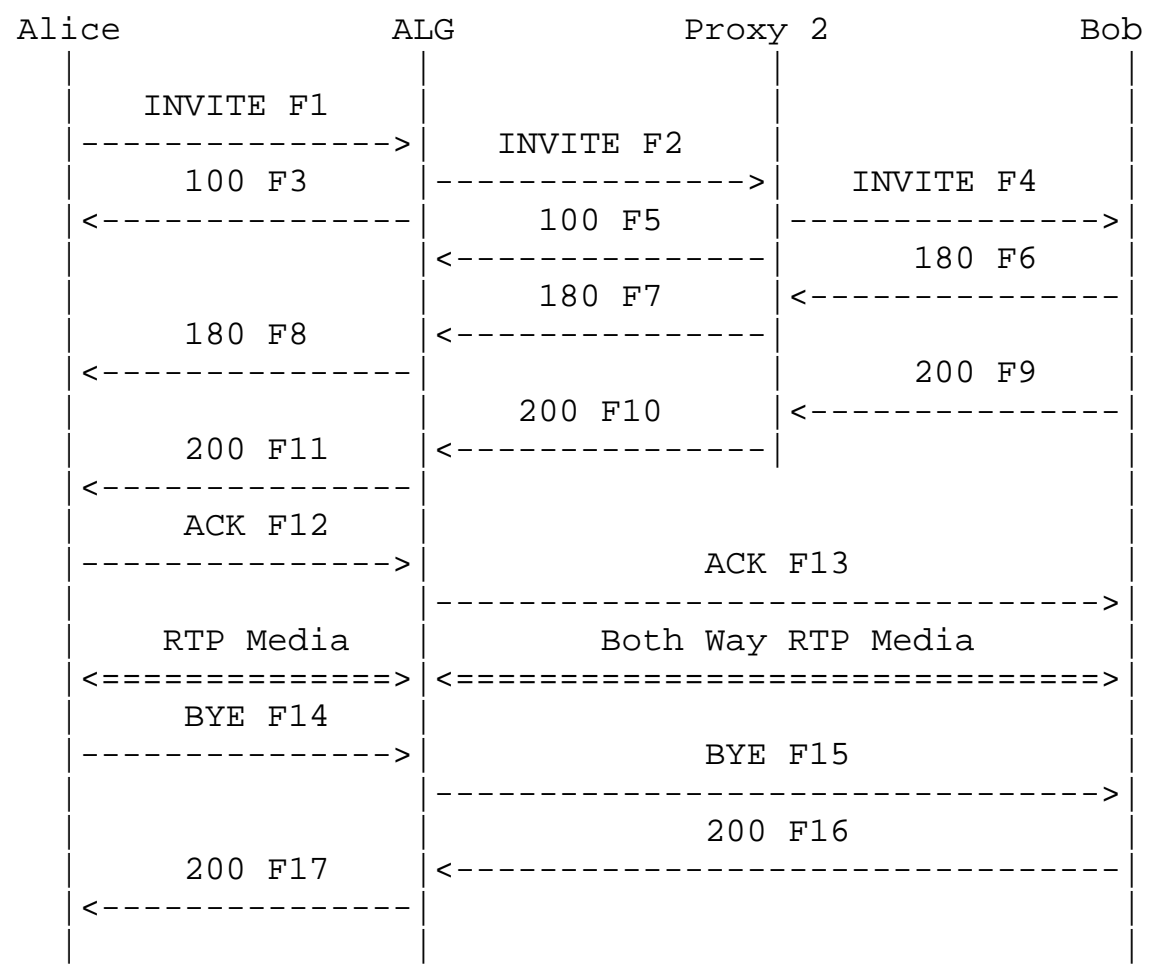

Alice completes a call to Bob through a ALG (Application Layer Gateway) and a SIP Proxy. The routing through the ALG is accomplished using a pre-loaded Route header in the INVITE F1. Note that the media stream setup is not end-to-end - the ALG terminates both media streams and bridges them. This is done by the ALG modifying the SDP in the INVITE (F1) and 200 OK (F10) messages, and possibly any $18 x$ or ACK messages containing SDP.

In addition to firewall traversal, this Back-to-Back User Agent (B2BUA) could be used as part of an anonymizer service (in which all identifying information on Alice would be removed), or to perform codec media conversion, such as mu-law to A-law conversion of PCM on an international call.

Also note that Proxy 2 does not Record-Route in this call flow. 


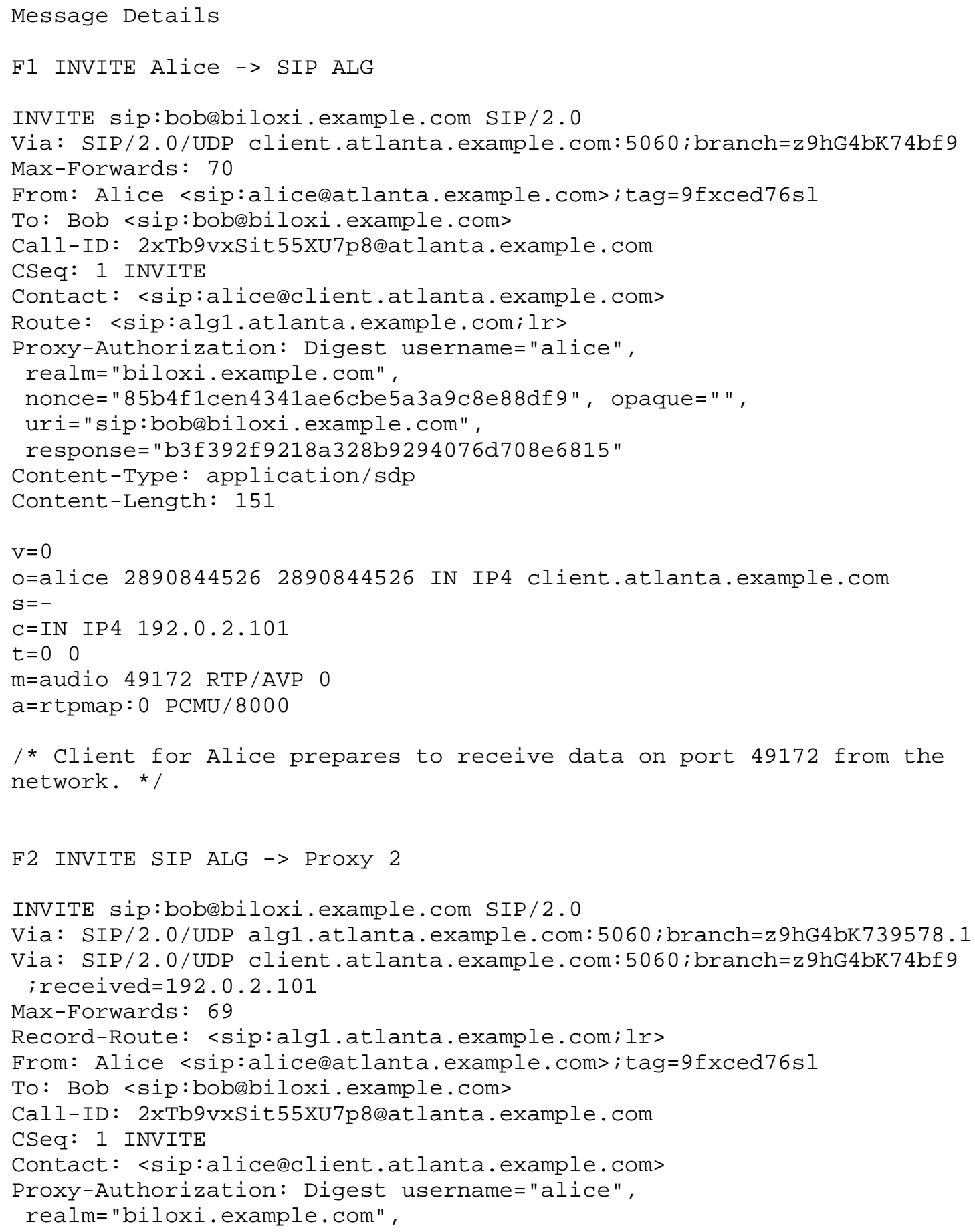




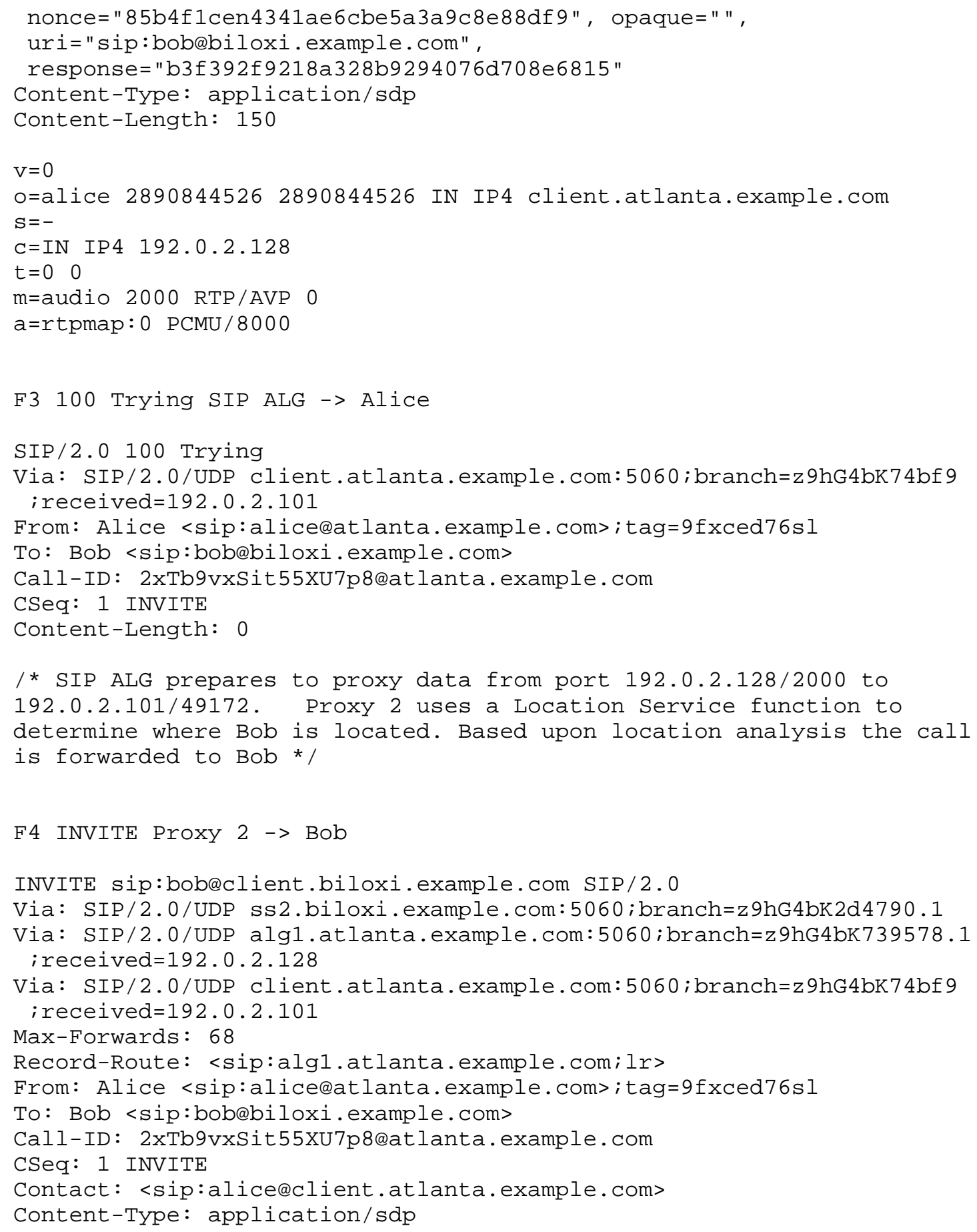




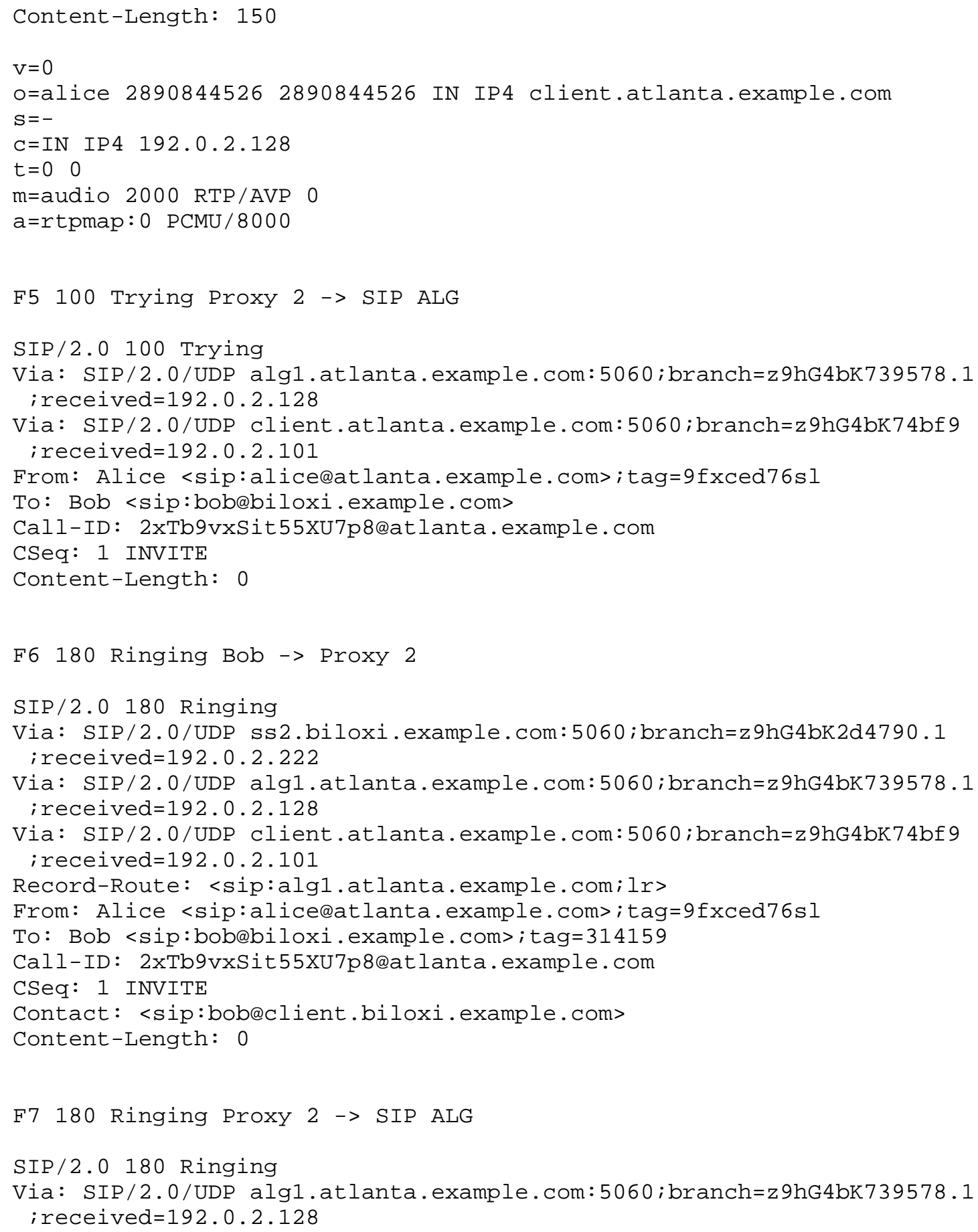


Via: SIP/2.0/UDP client.atlanta.example.com:5060; branch=z9hG4bK74bf 9 ; received=192.0.2.101

Record-Route: <sip:algl.atlanta.example.com;lr>

From: Alice <sip:alicelatlanta.example.com>; tag=9fxced76sl

To: Bob <sip:bobebiloxi.example. com>; tag=314159

Call-ID: 2xTb9vxSit55xU7p8@atlanta.example.com

CSeq: 1 INVITE

Contact: <sip:bob@client.biloxi.example.com>

Content-Length: 0

F8 180 Ringing SIP ALG $\rightarrow$ Alice

SIP/2.0 180 Ringing

Via: SIP/2.0/UDP client.atlanta.example.com:5060; branch=z9hG4bK74bf 9 ; received=192.0.2.101

Record-Route: <sip:alg1.atlanta.example.com;lr>

From: Alice <sip:alicelatlanta.example.com>; tag=9fxced76sl

To: Bob <sip:bob@biloxi.example.com>; tag=314159

Call-ID: 2xTb9vxSit55XU7p8eatlanta.example.com

CSeq: 1 INVITE

Contact: <sip:bob@client.biloxi.example.com>

Content-Length: 0

F9 200 OK Bob $->$ Proxy 2

SIP/2.0 200 OK

Via: SIP/2.0/UDP ss2.biloxi.example.com:5060; branch=z9hG4bK2d4790.1 ; received=192.0.2.222

Via: SIP/2.0/UDP alg1.atlanta.example.com:5060; branch=z9hG4bK739578.1 ; received $=192.0 .2 .128$

Via: SIP/2.0/UDP client.atlanta. example.com:5060; branch=z9hG4bK74bf 9 ; received=192.0.2.101

Record-Route: <sip:algl.atlanta.example.com; lr>

From: Alice <sip:alicedatlanta.example.com>; tag=9fxced76sl

To: Bob <sip:bob@biloxi.example.com>; tag=314159

Call-ID: 2xTb9vxSit55XU7p8datlanta.example.com

CSeq: 1 INVITE

Contact: <sip:bobeclient.biloxi.example.com>

Content-Type: application/sdp

Content-Length: 147

$\mathrm{V}=0$

o=bob 28908445272890844527 IN IP4 client.biloxi.example.com

$\mathrm{S}=-$

$\mathrm{C}=\mathrm{IN}$ IP 4 192.0.2.201

$t=0 \quad 0$

Johnston, et al.

Best Current Practice

[Page 50] 


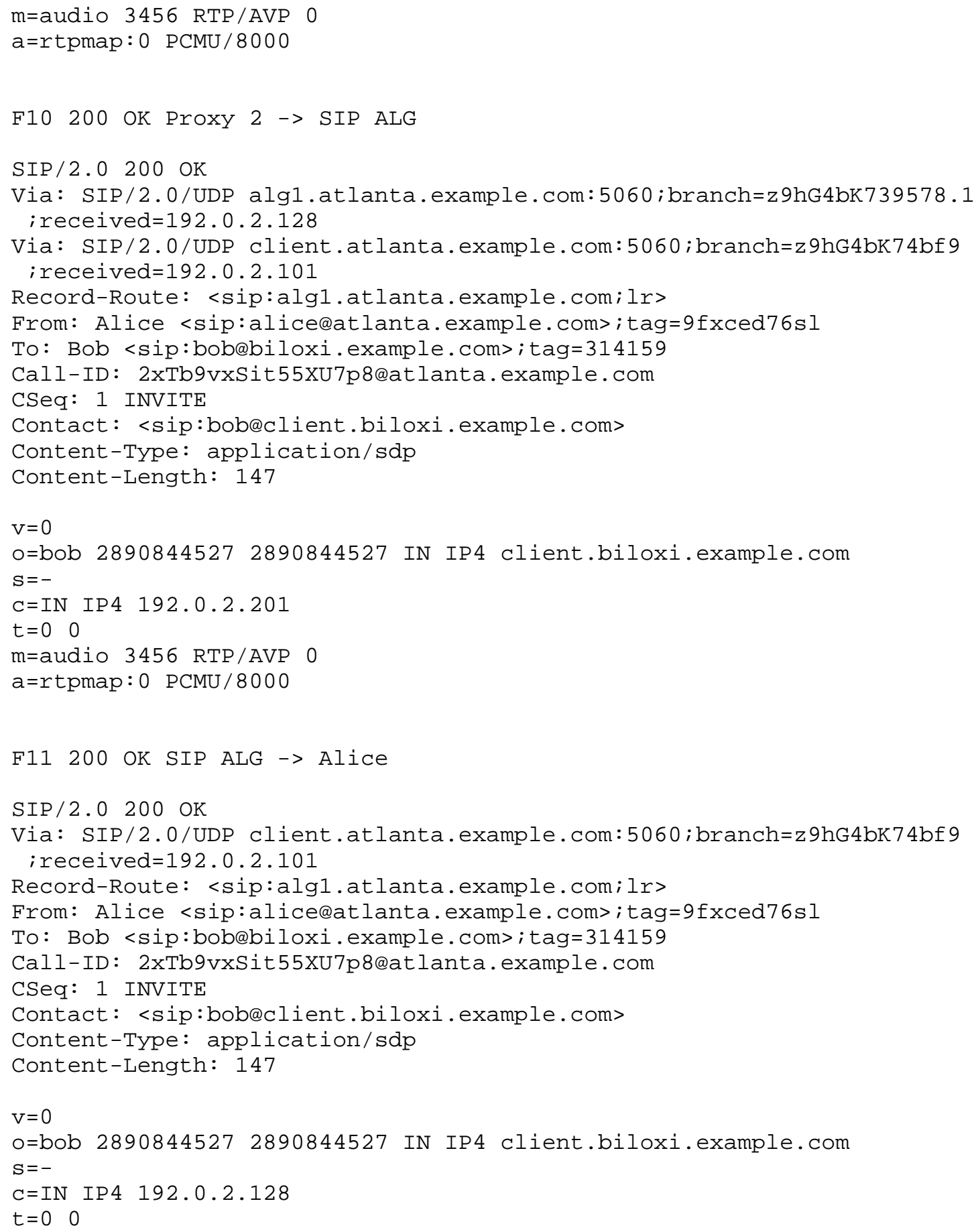




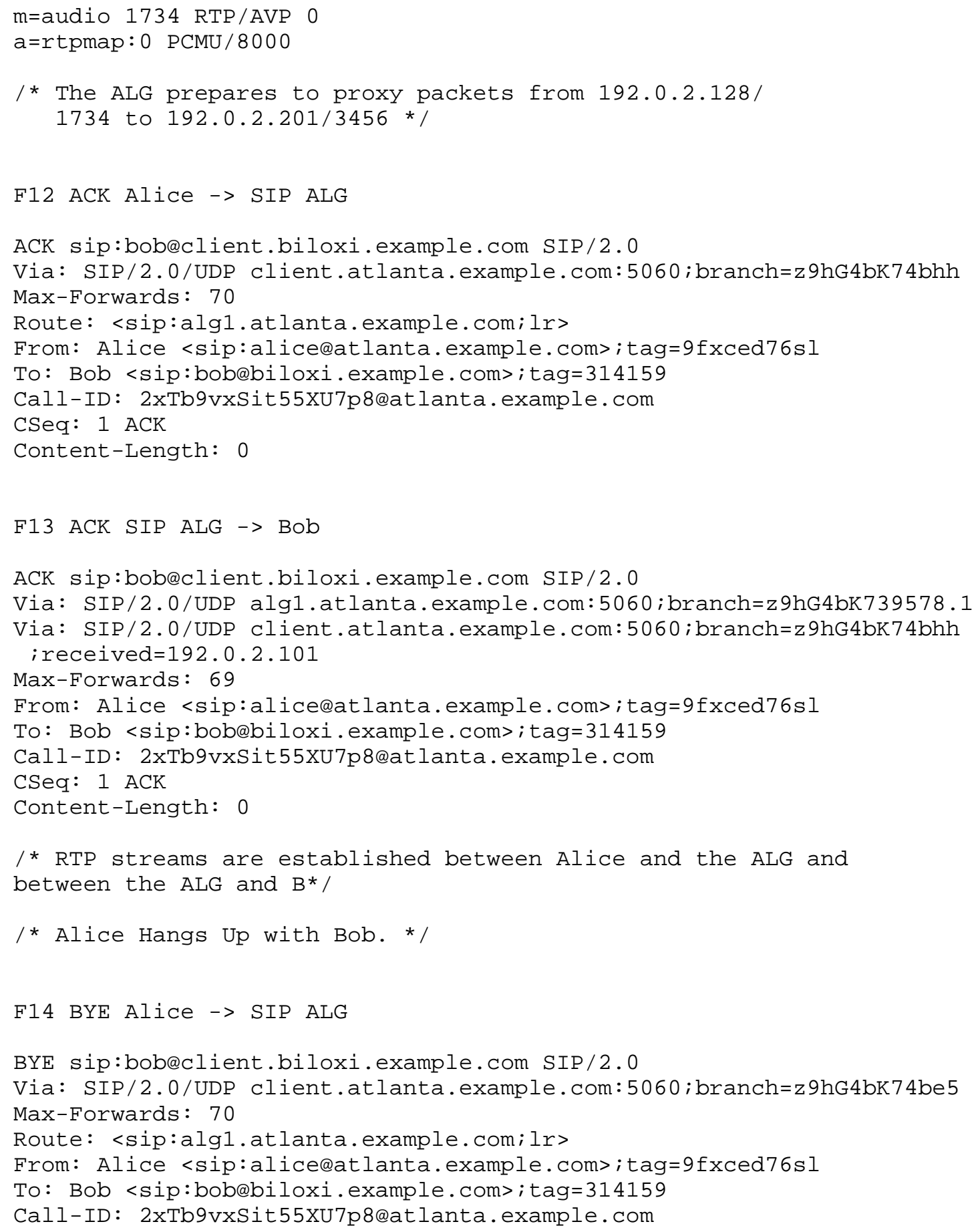




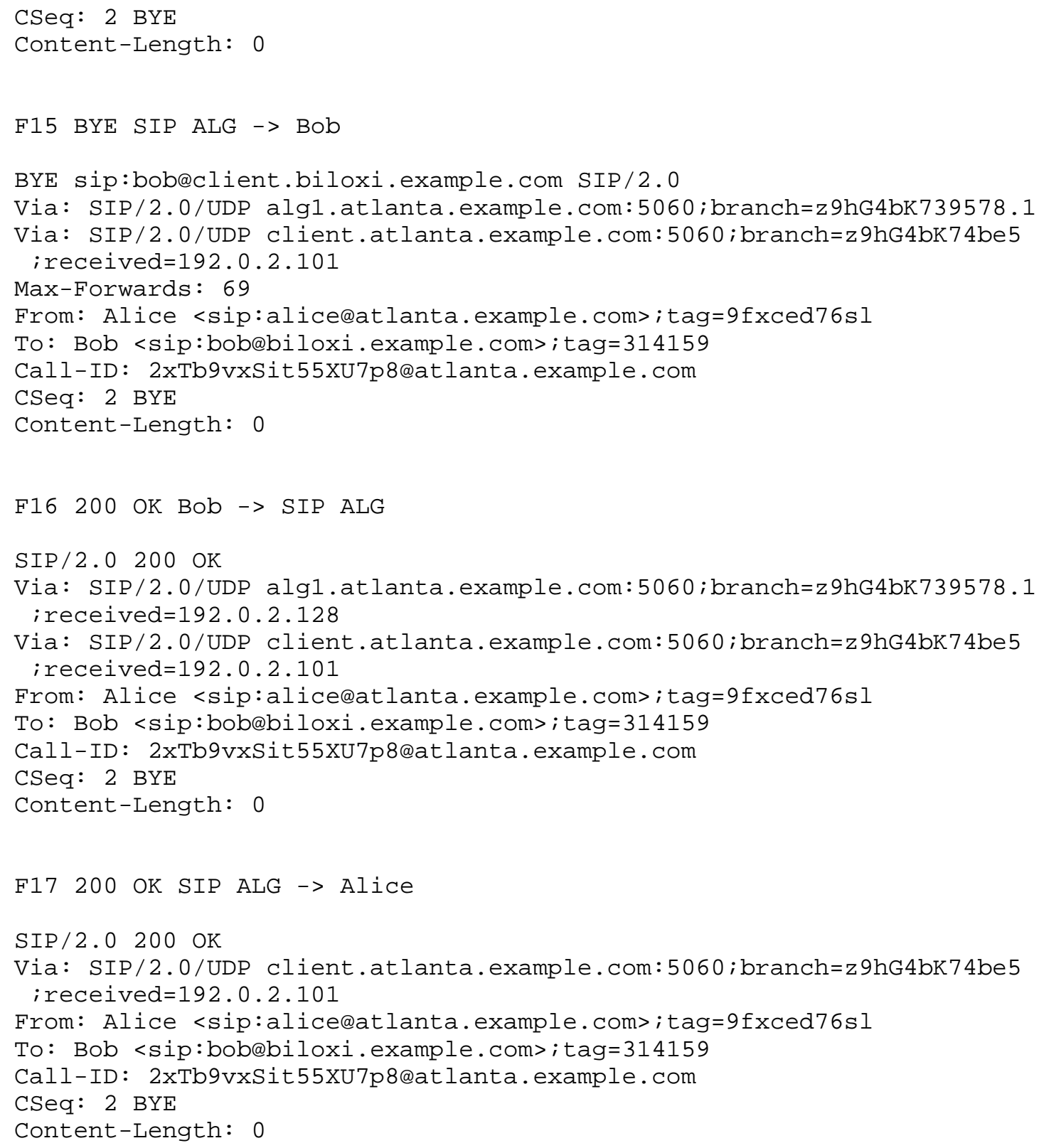


3.6. Session via Redirect and Proxy Servers with SDP in ACK

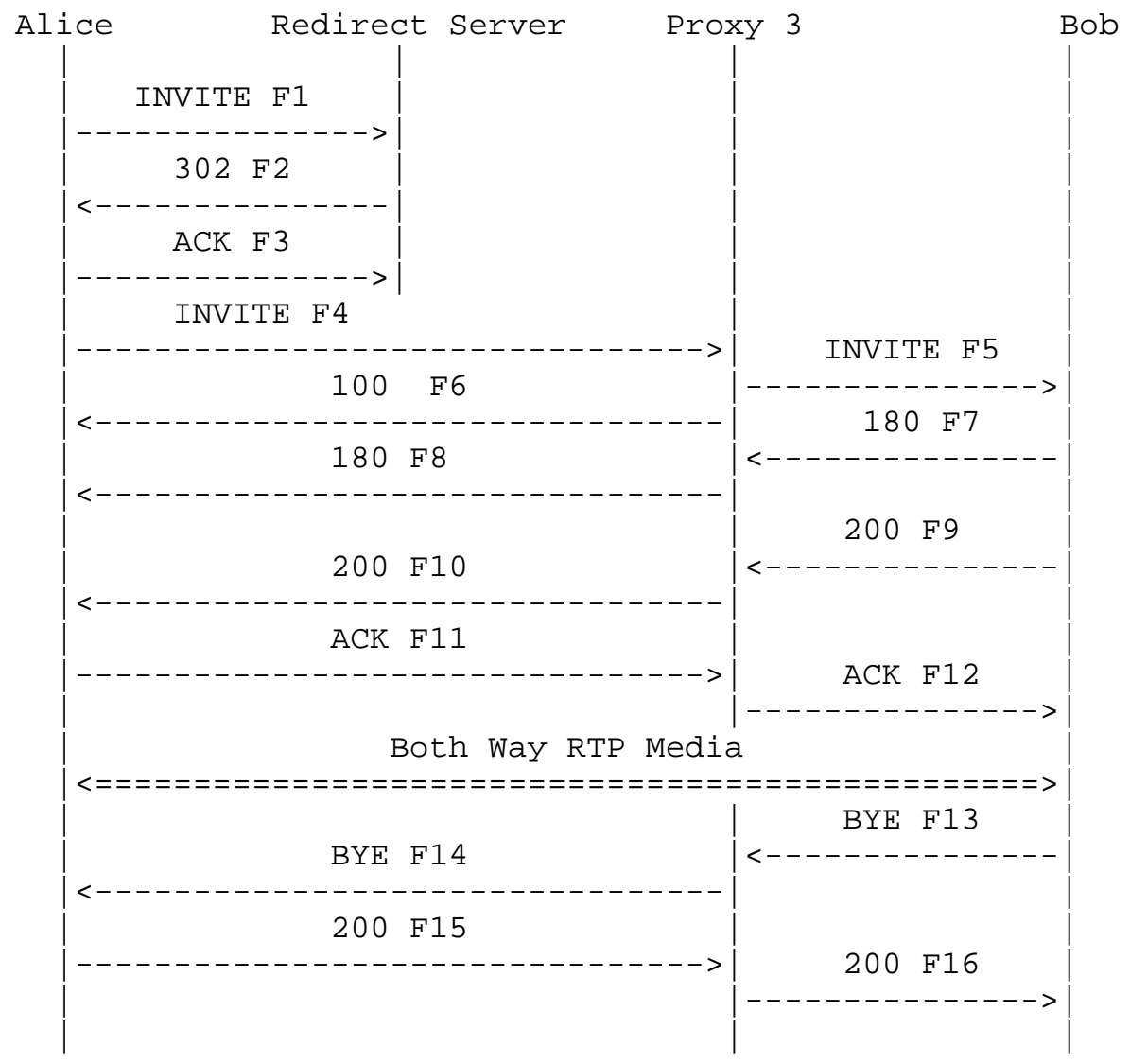

In this scenario, Alice places a call to Bob using first a Redirect server then a Proxy server. The INVITE message is first sent to the Redirect Server. The Server returns a 302 Moved Temporarily response (F2) containing a contact header with Bob's current SIP address. Alice then generates a new INVITE and sends to Bob via the Proxy Server and the call proceeds normally. In this example, no SDP is present in the INVITE, so the SDP is carried in the ACK message.

The call is terminated when Bob sends a BYE message. 


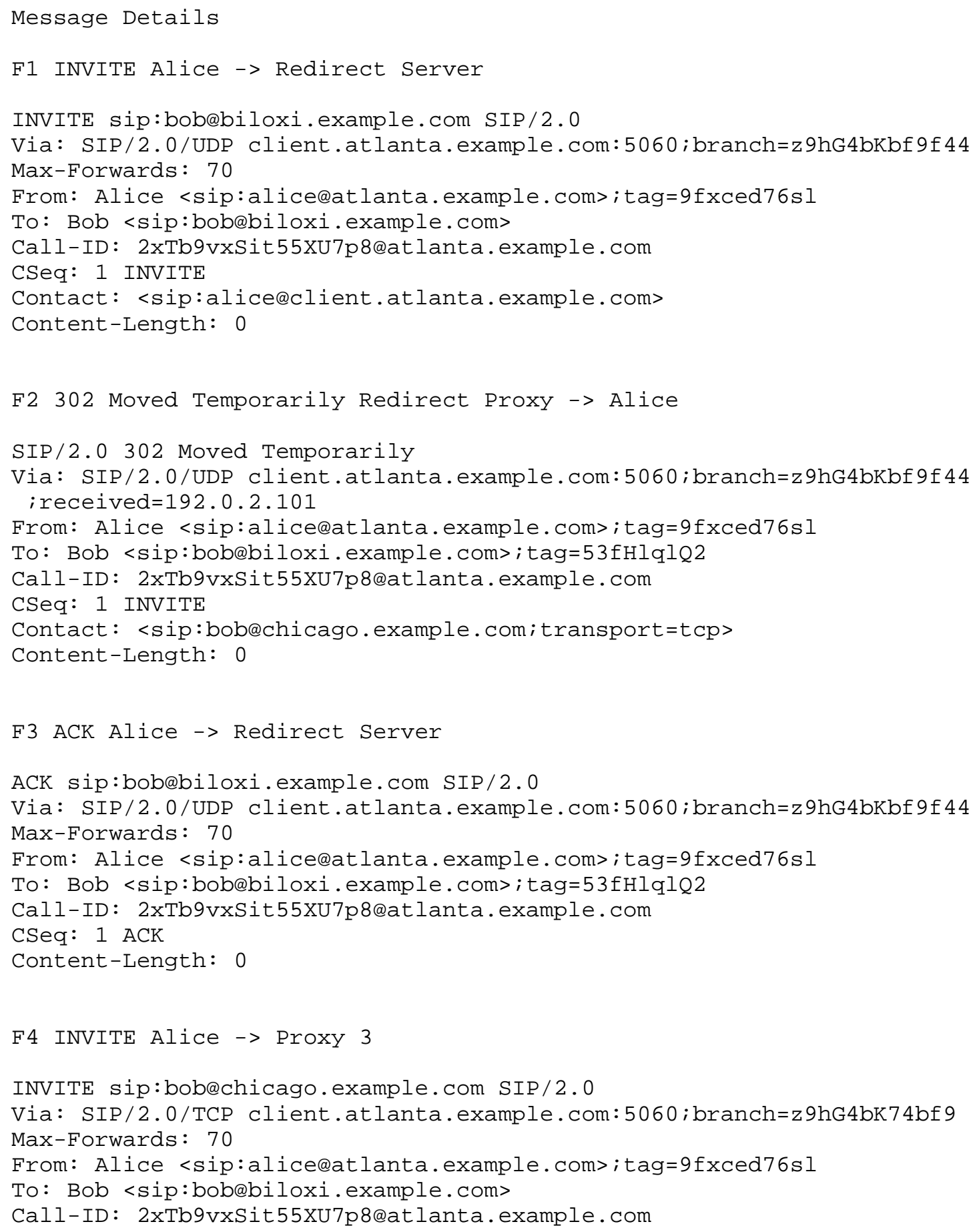




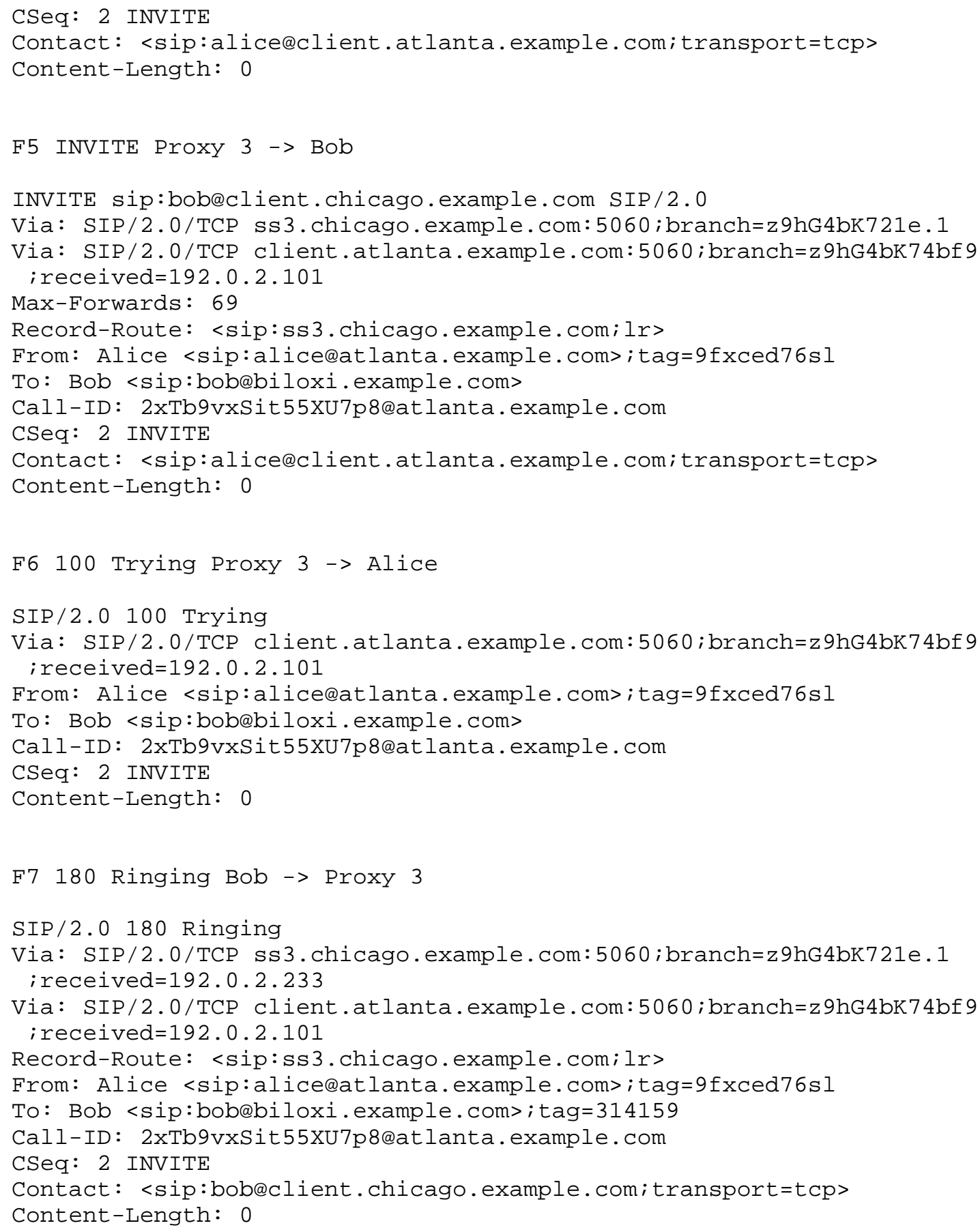


F8 180 Ringing Proxy $3->$ Alice

SIP/2.0 180 Ringing

Via: SIP/2.0/TCP client.atlanta. example.com:5060; branch=z9hG4bK74bf 9 i received=192.0.2.101

Record-Route: <sip:ss3.chicago.example.com;lr>

From: Alice <sip:alicelatlanta.example.com>; tag=9fxced76sl

To: Bob <sip:bob@biloxi.example.com>; tag=314159

Call-ID: 2xTb9vxSit55XU7p8eatlanta.example.com

CSeq: 2 INVITE

Contact: <sip:bobeclient. chicago.example.com; transport=tcp>

Content-Length: 0

F9 200 OK Bob $->$ Proxy 3

SIP $/ 2.0200$ OK

Via: SIP/2.0/TCP ss3. chicago.example.com:5060; branch=z9hG4bK721e.1 ; received=192.0.2.233

Via: SIP/2.0/TCP client.atlanta. example.com:5060; branch=z9hG4bK74bf 9 ; received=192.0.2.101

Record-Route: <sip:ss3.chicago.example.com;lr>

From: Alice <sip:alicelatlanta.example.com>; tag=9fxced76sl

To: Bob <sip:bob@biloxi.example.com>; tag=314159

Call-ID: 2xTb9vxSit55XU7p8eatlanta.example.com

CSeq: 2 INVITE

Contact: <sip:bobeclient.chicago.example.com; transport=tcp>

Content-Type: application/sdp

Content-Length: 148

$\mathrm{V}=0$

o=bob 28908445272890844527 IN IP4 client.chicago.example.com

$\mathrm{s}=-$

$\mathrm{C}=\mathrm{IN}$ IP 4 192.0.2.100

$t=0 \quad 0$

m=audio 3456 RTP/AVP 0

a=rtpmap: 0 PCMU/8000

F10 200 OK Proxy -> Alice

SIP/2.0 200 OK

Via: SIP/2.0/TCP client. atlanta.example.com:5060; branch=z9hG 4 bK 74 bf 9 ; received=192.0.2.101

Record-Route: <sip:ss3.chicago.example.com;lr>

From: Alice <sip:alicelatlanta.example.com>; tag=9fxced76sl

To: Bob <sip:bob@biloxi.example.com>; tag=314159 


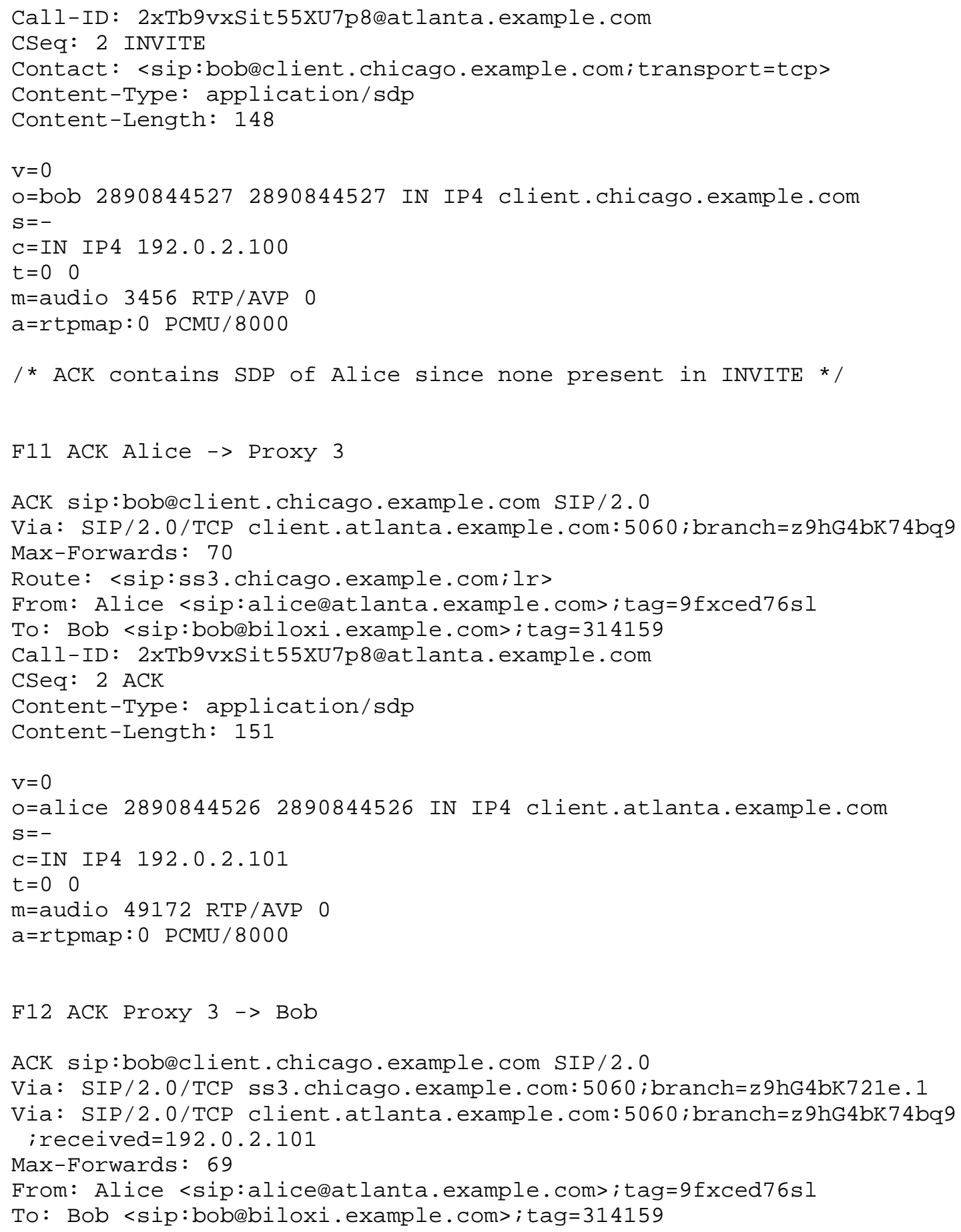




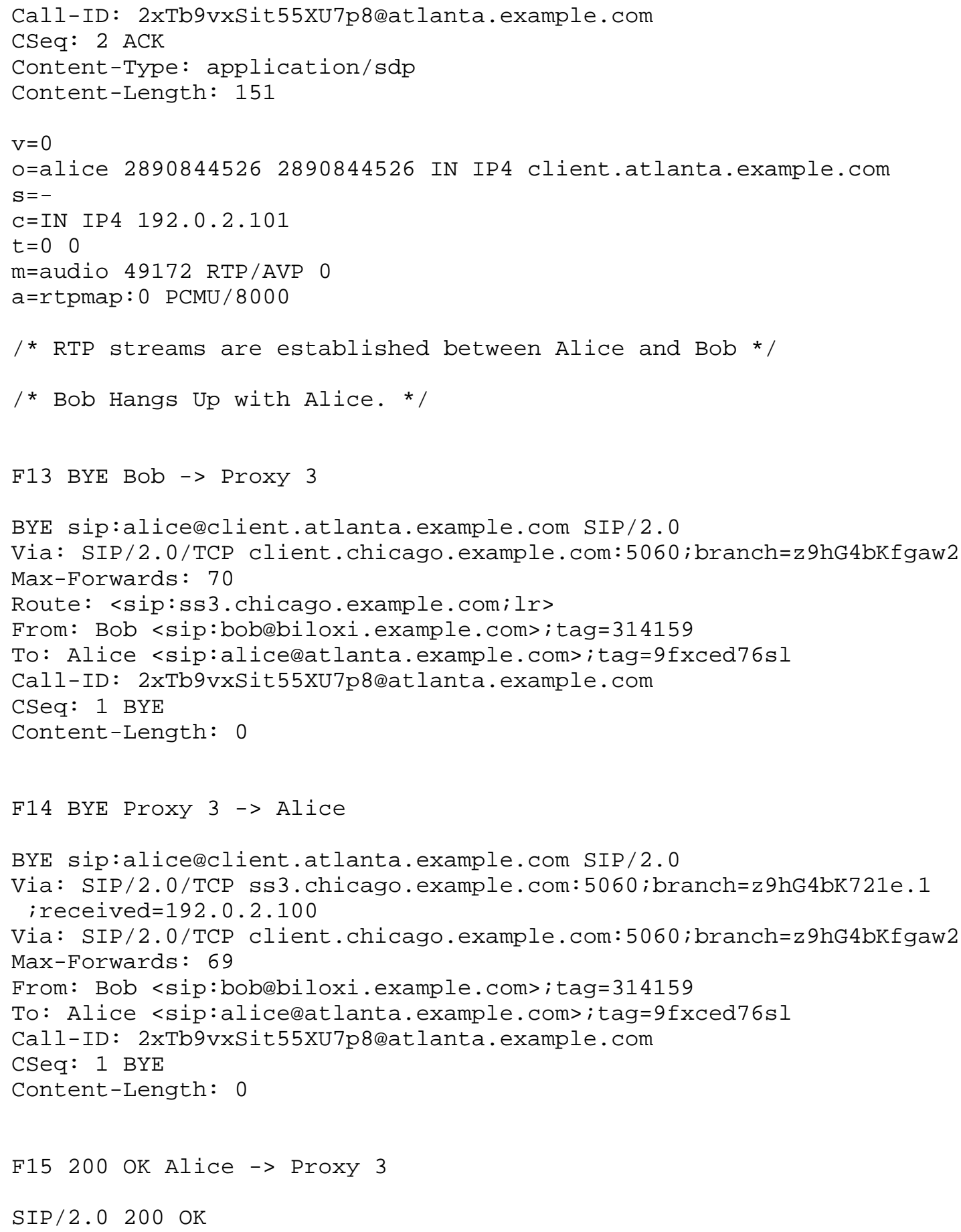


Via: SIP/2.0/TCP ss3. chicago. example.com:5060; branch=z9hG4bK721e.1 ; received $=192.0 .2 .233$

Via: SIP/2.0/TCP client. chicago.example.com:5060;branch=z9hG4bKfgaw2 ; received=192.0.2.100

From: Bob <sip:bobebiloxi.example.com>; tag=314159

To: Alice <sip:alicelatlanta.example.com>; tag=9fxced76sl

Call-ID: 2xTb9vxSit55XU7p8@atlanta.example.com

CSeq: 1 BYE

Content-Length: 0

F16 200 OK Proxy $3->$ Bob

$\mathrm{SIP} / 2.0200 \mathrm{OK}$

Via: SIP/2.0/TCP client. chicago.example.com:5060;branch=z9hG4bKfgaw2 ; received=192.0.2.100

From: Bob <sip:bob@biloxi.example.com>; tag=314159

To: Alice <sip:alicelatlanta.example.com>;tag=9fxced76sl

Call-ID: 2xTb9vxSit55XU7p8eatlanta.example.com

CSeq: 1 BYE

Content-Length: 0 


\subsection{Session with re-INVITE (IP Address Change)}

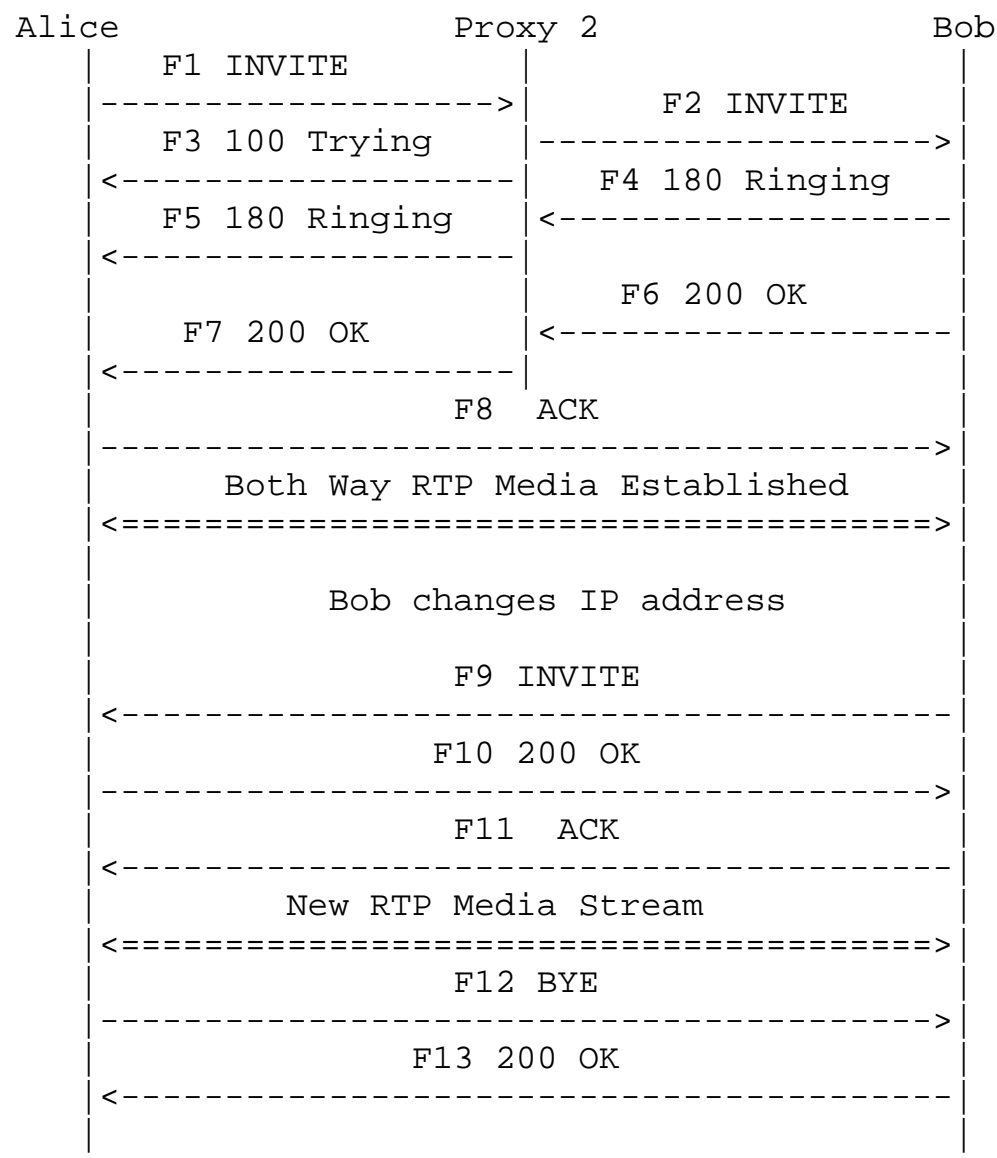

This example shows a session in which the media changes midway through the session. When Bob's IP address changes during the session, Bob sends a re-INVITE containing a new Contact and SDP (version number incremented) information to A. In this flow, the proxy does not Record-Route so is not in the SIP messaging path after the initial exchange. 


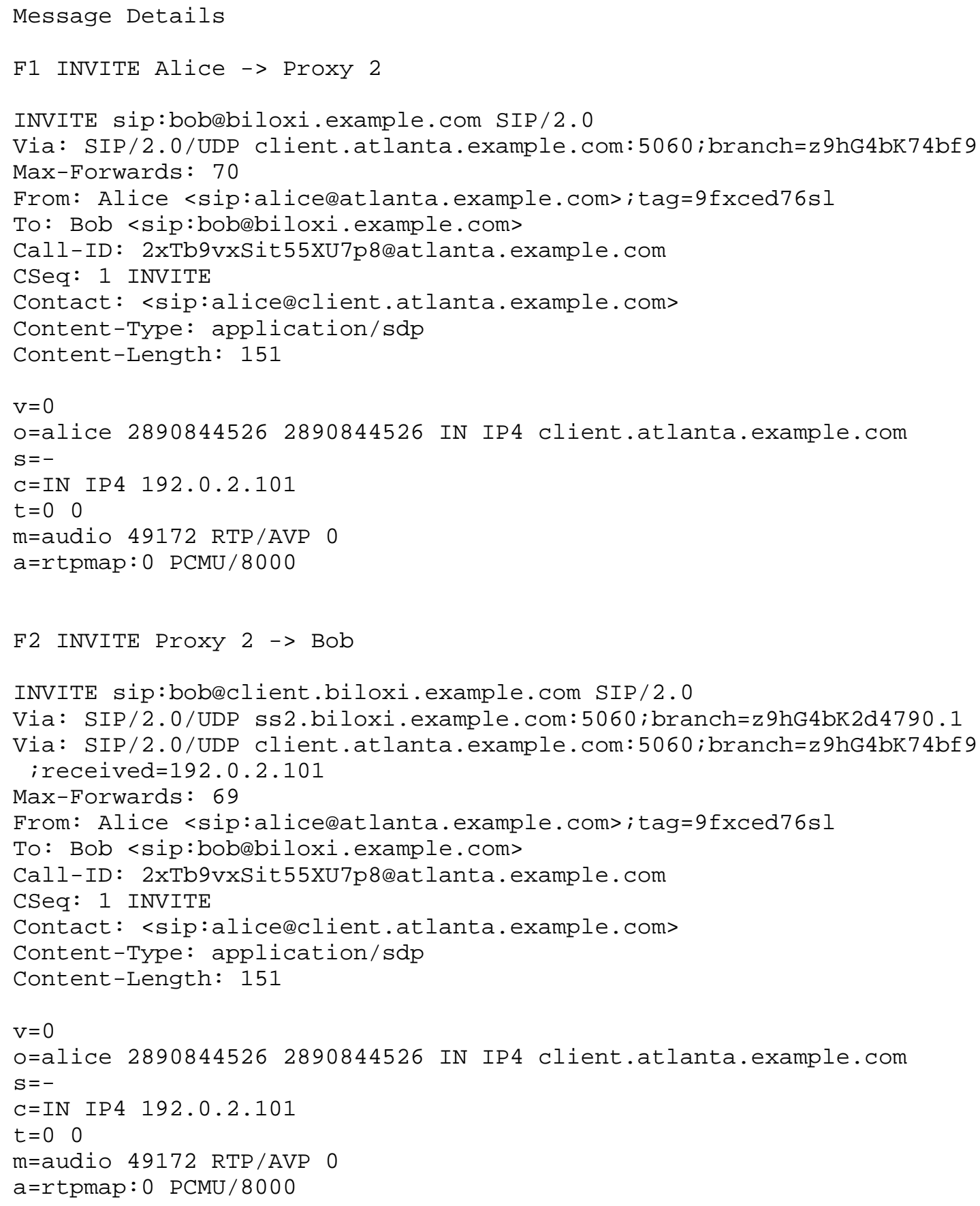


F3 100 Trying Proxy $2 \rightarrow$ Alice

SIP/2.0 100 Trying

Via: SIP/2.0/UDP client.atlanta.example.com:5060; branch=z9hG4bK74bf 9 i received $=192.0 .2 .101$

From: Alice <sip:alicedatlanta.example.com>; tag=9fxced76sl

To: Bob <sip:bobebiloxi.example.com>

Call-ID: 2xTb9vxSit55XU7p8@atlanta.example.com

CSeq: 1 INVITE

Content-Length: 0

F4 180 Ringing Bob $\rightarrow$ Proxy 2

SIP/2.0 180 Ringing

Via: SIP/2.0/UDP ss2.biloxi.example.com:5060; branch=z9hG4bK2d4790.1 ; received $=192.0 .2 .222$

Via: SIP/2.0/UDP client.atlanta. example.com:5060; branch=z9hG4bK74bf 9 ireceived=192.0.2.101

From: Alice <sip:alicedatlanta.example.com>; tag=9fxced76sl

To: Bob <sip:bob@biloxi.example.com>; tag=314159

Call-ID: 2xTb9vxSit55XU7p8@atlanta.example.com

CSeq: 1 INVITE

Contact: <sip:bob@client.biloxi.example.com>

Content-Length: 0

F5 180 Ringing Proxy $2->$ Alice

SIP/2.0 180 Ringing

Via: SIP/2.0/UDP client.atlanta.example.com:5060; branch=z9hG4bK74bf 9 ; received=192.0.2.101

From: Alice <sip:alicelatlanta.example.com>; tag=9fxced76sl

To: Bob <sip:bobabiloxi.example.com>; tag=314159

Call-ID: 2xTb9vxSit55XU7p8@atlanta.example.com

CSeq: 1 INVITE

Contact: <sip:bobeclient.biloxi.example.com>

Content-Length: 0

F6 200 OK Bob -> Proxy 2

$\mathrm{SIP} / 2.0200 \mathrm{OK}$

Via: SIP/2.0/UDP ss2.biloxi.example.com:5060; branch=z9hG4bK2d4790.1 ; received $=192.0 .2 .222$

Via: SIP/2.0/UDP client.atlanta.example.com:5060; branch=z9hG4bK74bf 9 ; received=192.0.2.101

From: Alice <sip:alicelatlanta.example.com>; tag=9fxced76sl 


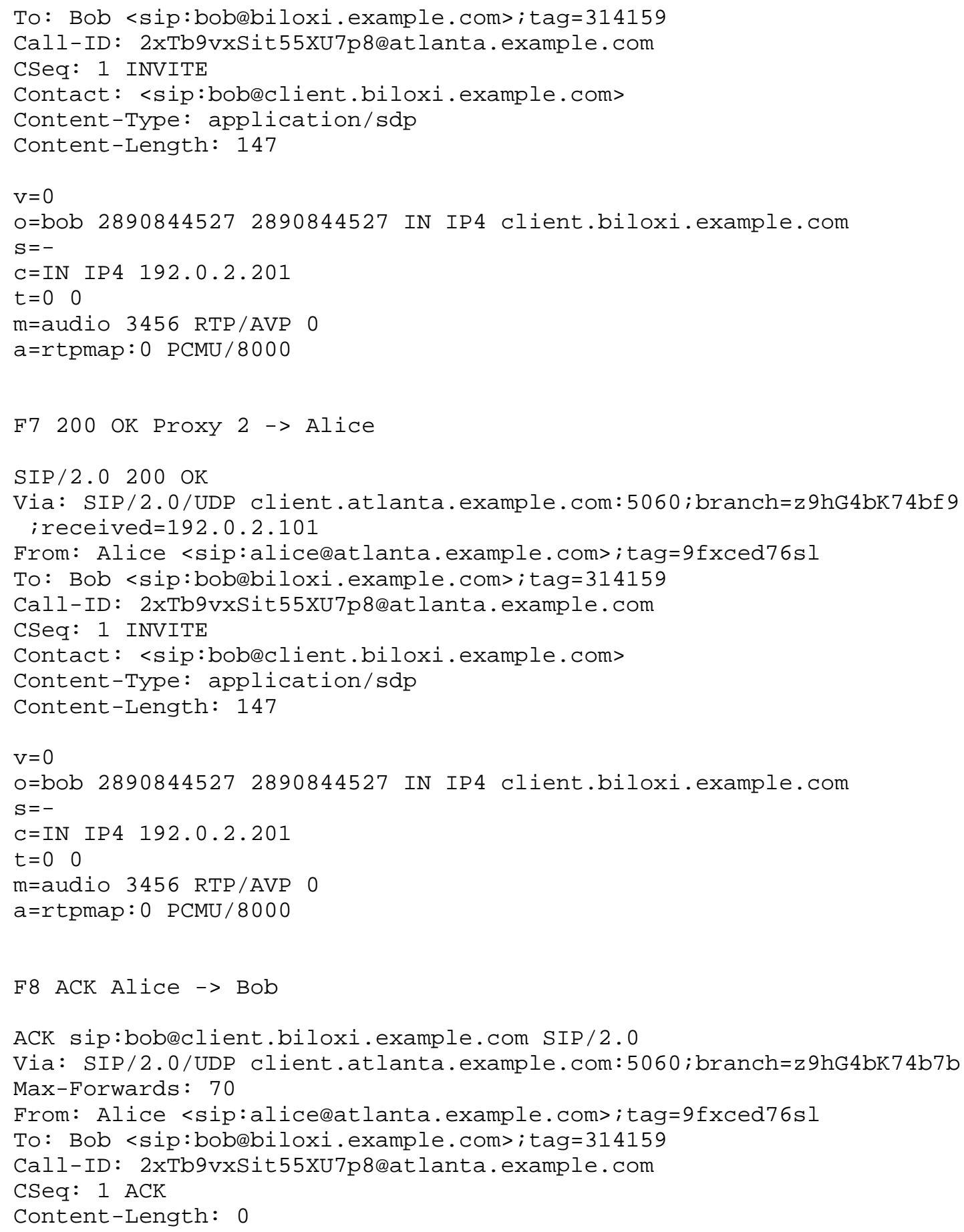




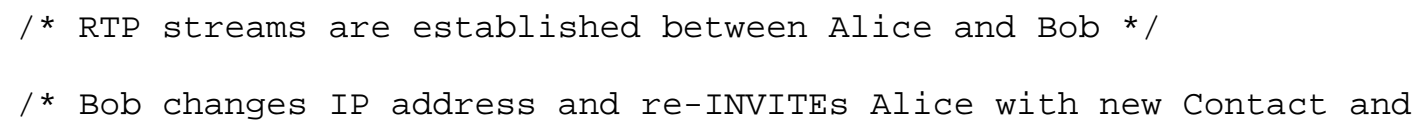




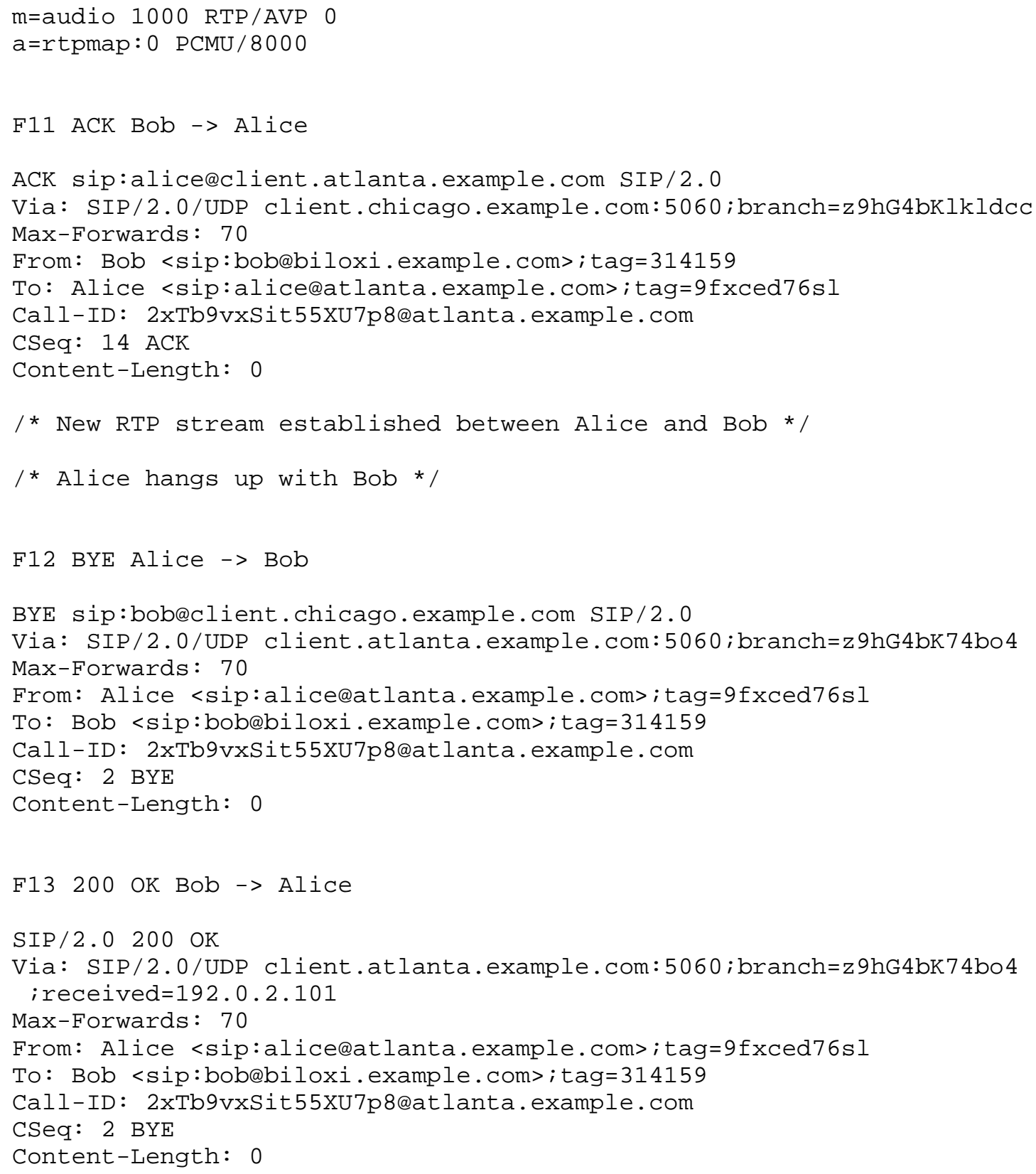




\subsection{Unsuccessful No Answer}

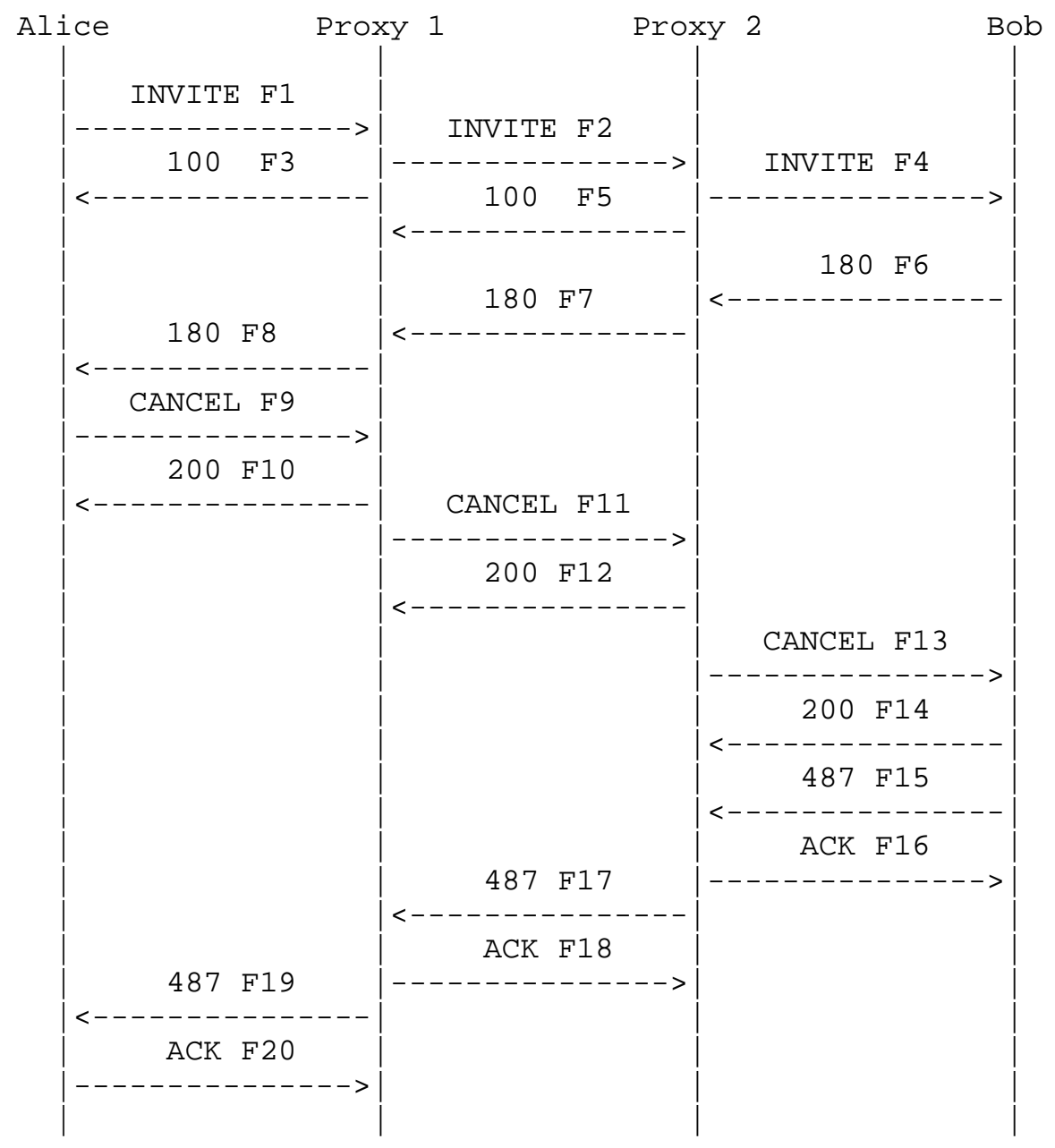

In this scenario, Alice gives up on the call before Bob answers (sends a 200 OK response). Alice sends a CANCEL (F9) since no final response had been received from Bob. If a 200 OK to the INVITE had crossed with the CANCEL, Alice would have sent an ACK then a BYE to Bob in order to properly terminate the call.

Note that the CANCEL message is acknowledged with a 200 OK on a hop by hop basis, rather than end to end. 


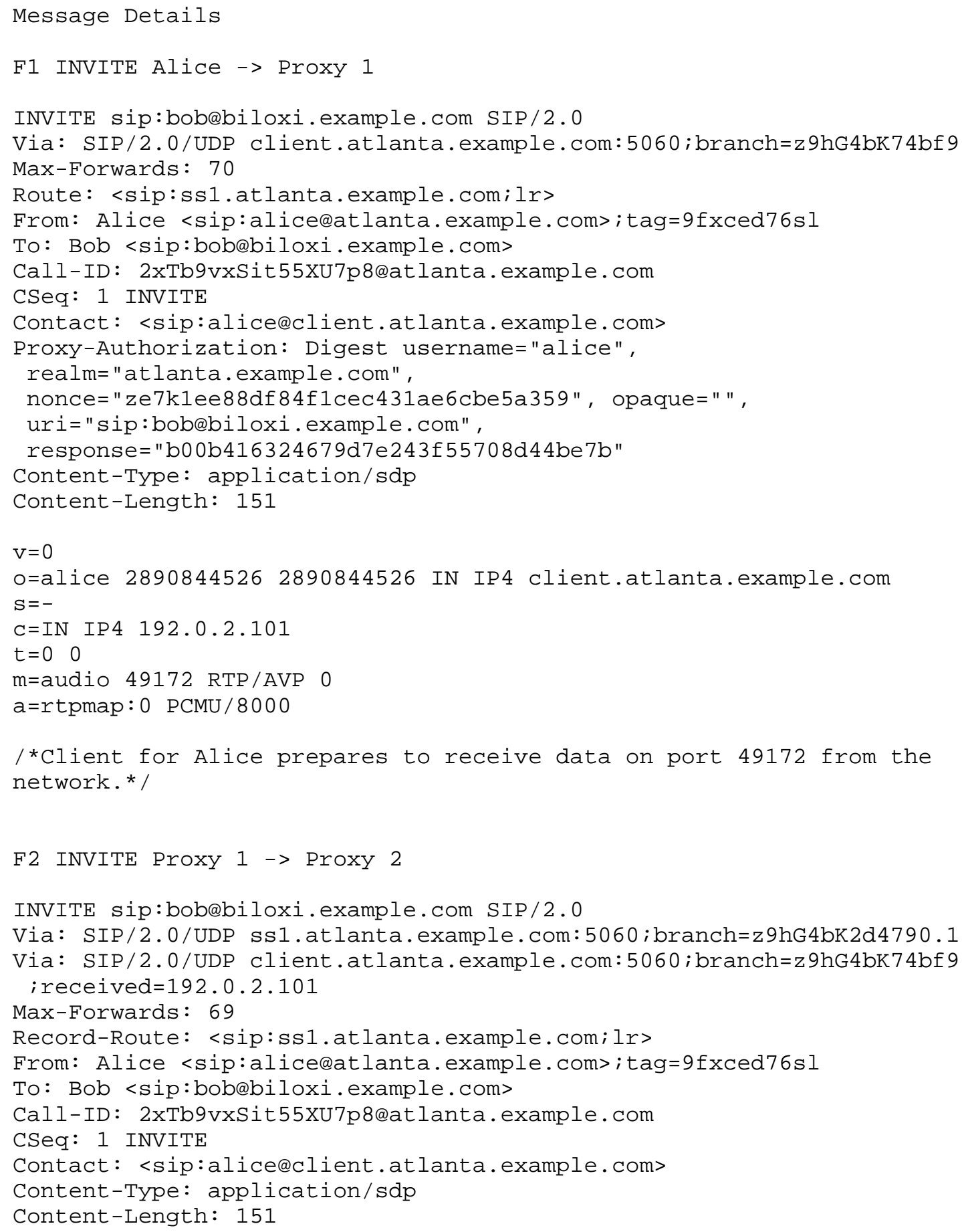




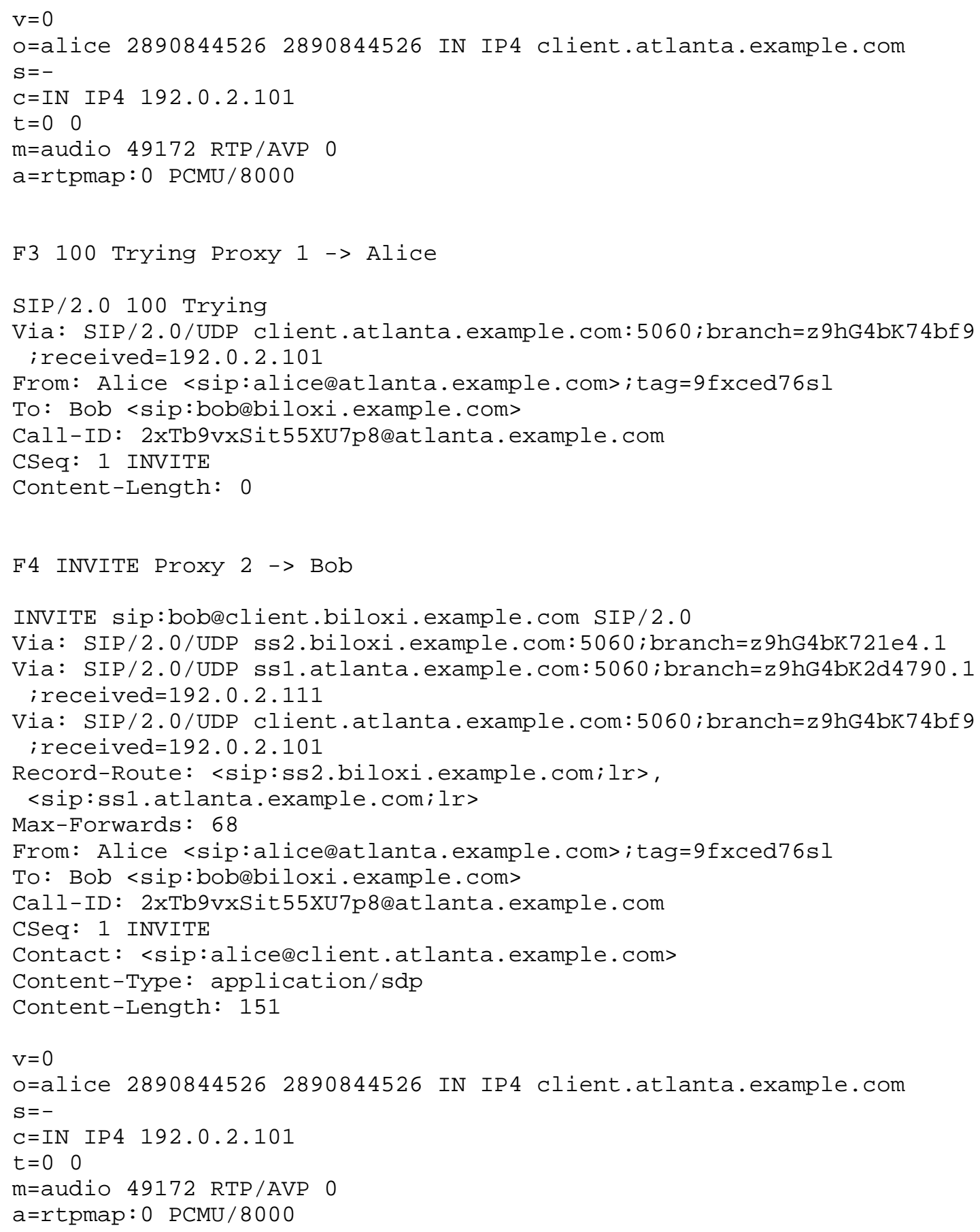




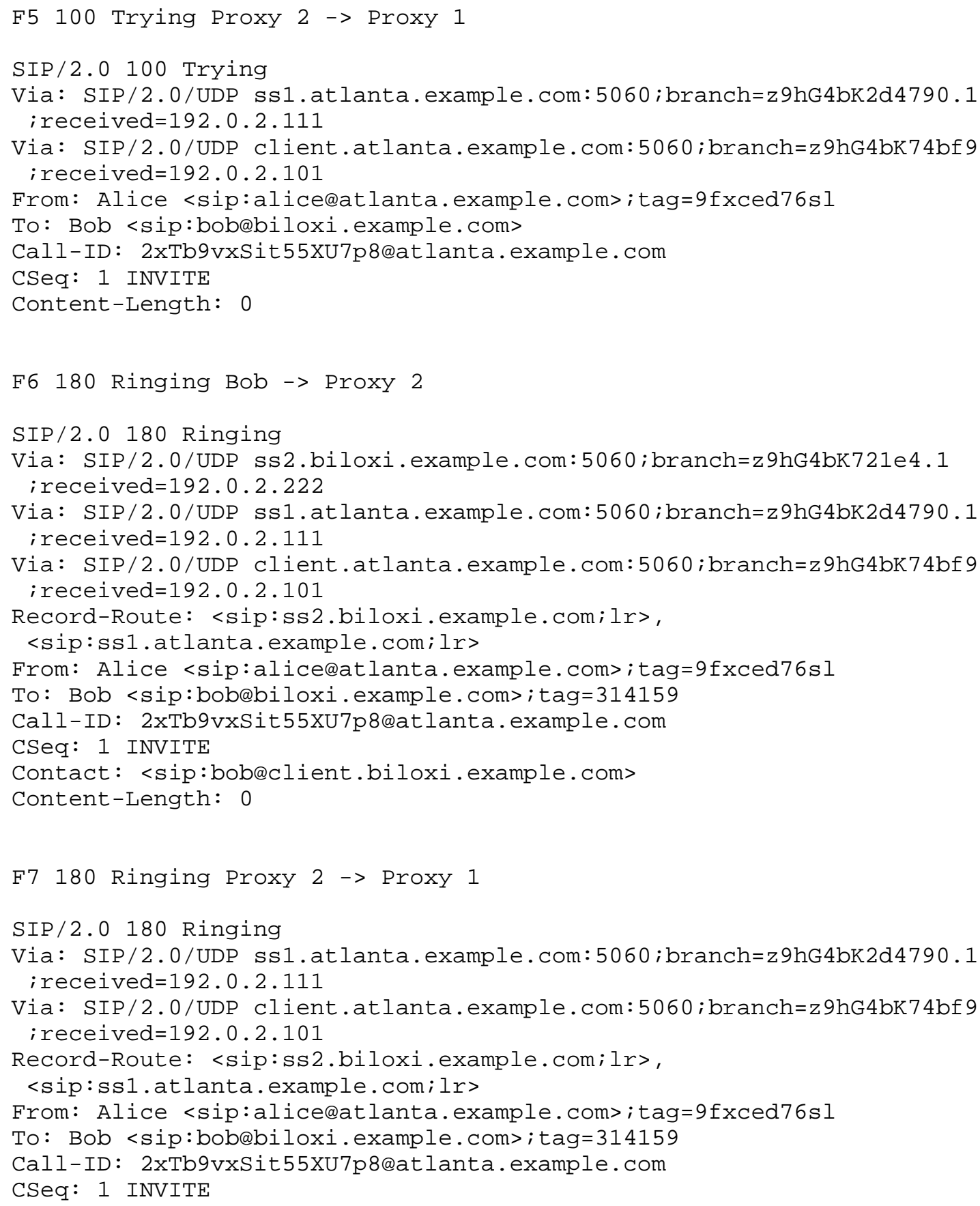




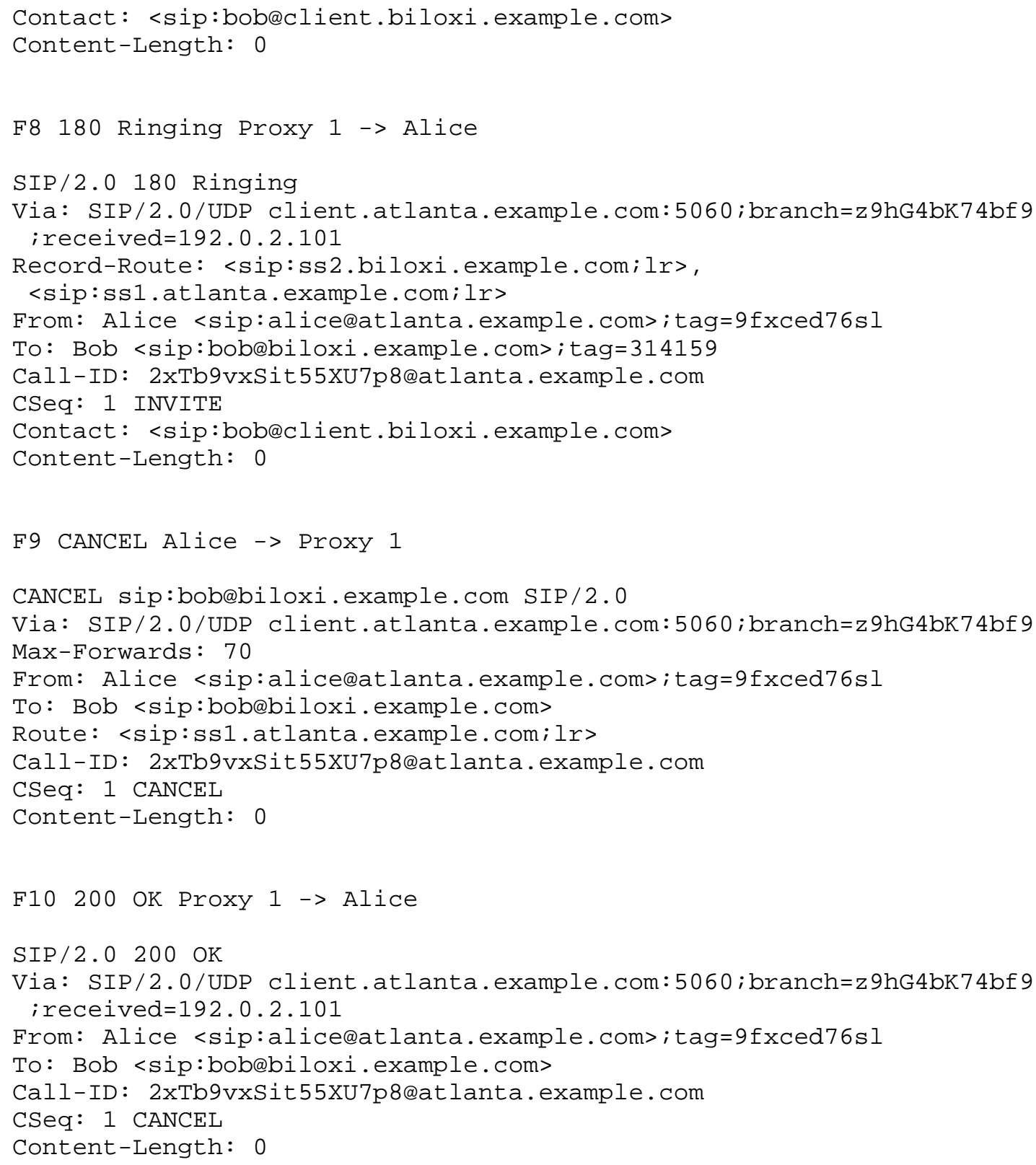




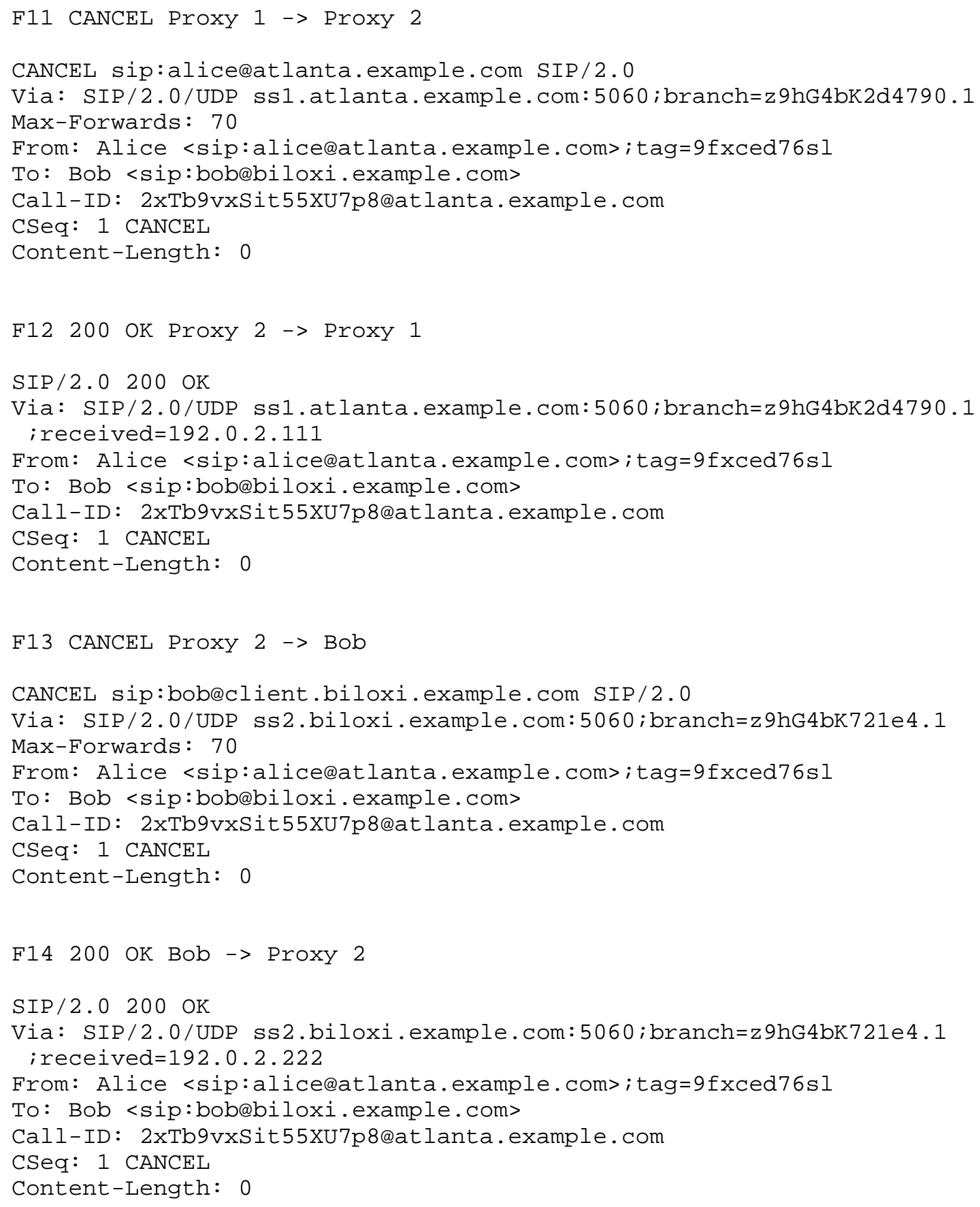




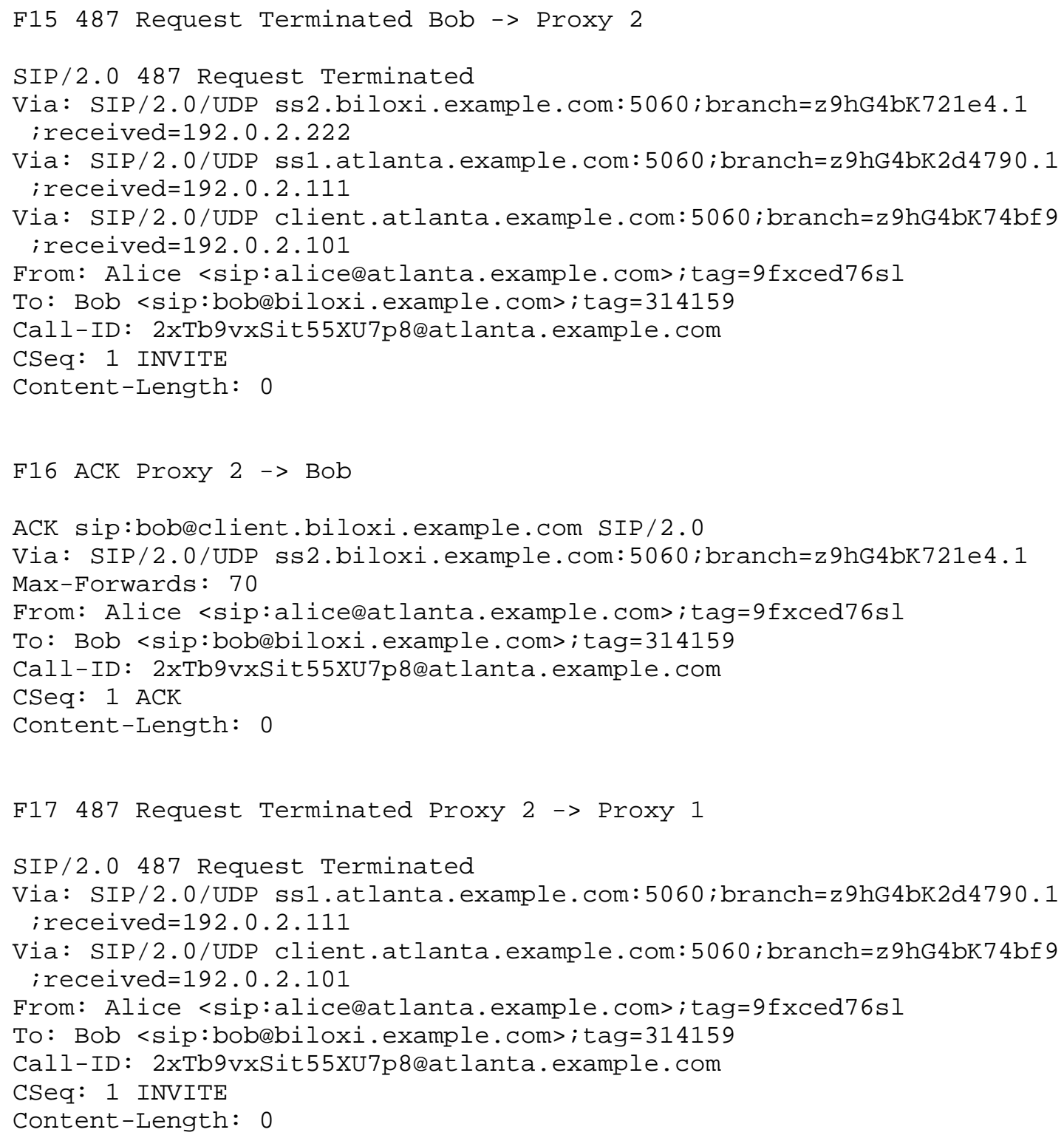




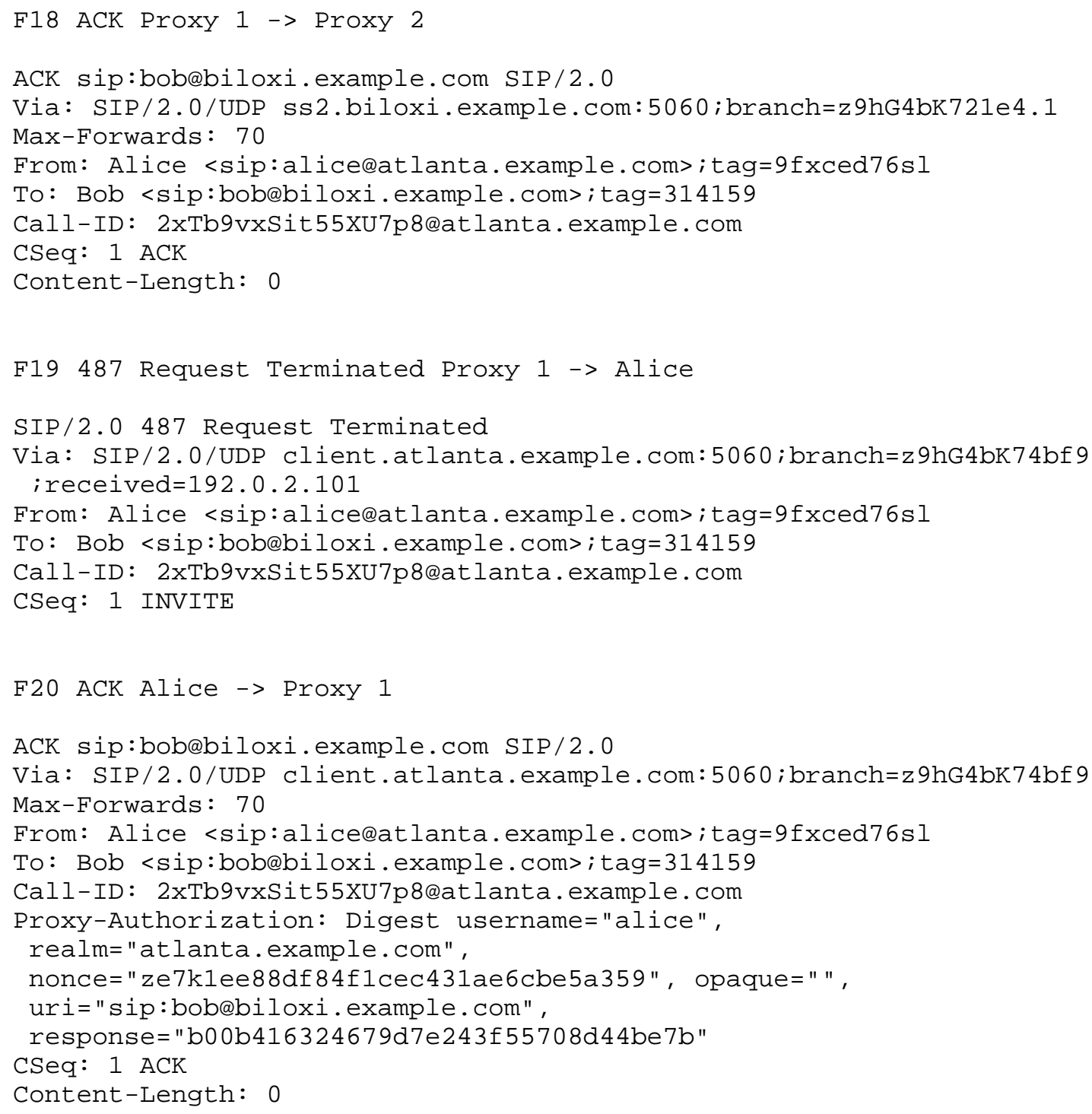


3.9. Unsuccessful Busy

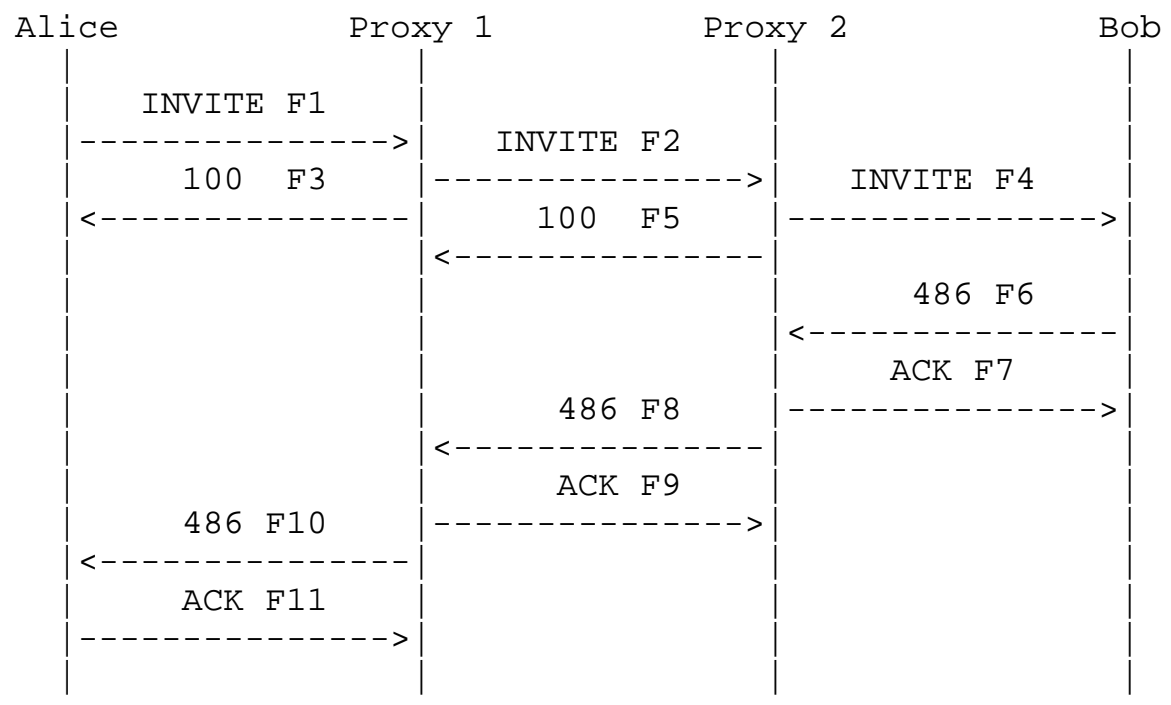

In this scenario, Bob is busy and sends a 486 Busy Here response to Alice's INVITE. Note that the non-2xx response is acknowledged on a hop-by-hop basis instead of end-to-end. Also note that many SIP UAS will not return a 486 response, as they have multiple line and other features.

Message Details

F1 INVITE Alice $->$ Proxy 1

INVITE sip:bob@biloxi.example.com SIP/2.0

Via: SIP/2.0/TCP client.atlanta. example.com:5060; branch=z 9 hG $4 \mathrm{bK} 74 \mathrm{bf} 9$ Max-Forwards: 70

Route: <sip:ssl.atlanta.example.com; lr>

From: Alice <sip:alicelatlanta. example.com>; tag=9fxced76sl

To: Bob <sip:boblbiloxi.example.com>

Call-ID: 2xTb9vxSit55XU7p8eatlanta.example.com

CSeq: 1 INVITE

Contact: <sip:alicedclient.atlanta.example.com;transport=tcp>

Proxy-Authorization: Digest username="alice", realm="atlanta.example.com", nonce="dc3a5ab2530aa93112cf5904ba7d88fa", opaque=" ", uri="sip:bob@biloxi.example.com", response="702138b27d869ac8741e10ec643d55be"

Content-Type: application/sdp

Content-Length: 151 


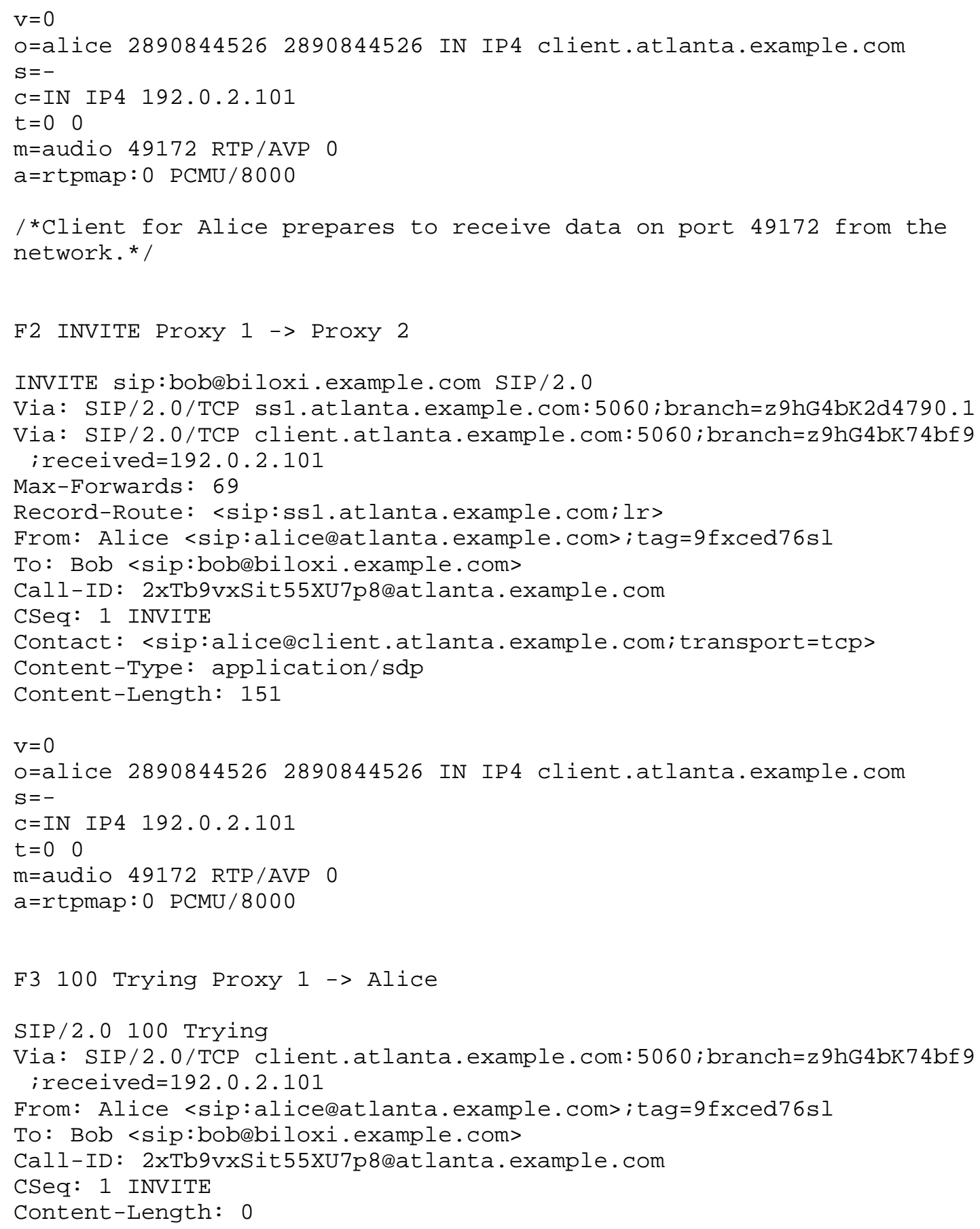


F4 INVITE Proxy $2 \rightarrow$ Bob

INVITE sip:bob@client.biloxi.example.com SIP/2.0

Via: SIP/2.0/TCP ss2.biloxi.example.com:5060; branch=z9hG4bK721e4.1

Via: SIP/2.0/TCP ss1.atlanta.example.com:5060; branch=z 9hG4bK2d4790.1 ireceived=192.0.2.111

Via: SIP/2.0/TCP client.atlanta.example.com:5060; branch=z9hG4bK74bf 9 ; received=192.0.2.101

Max-Forwards: 68

Record-Route: <sip:ss2.biloxi.example.com;lr>, <sip:ss1.atlanta.example.com; lr>

From: Alice <sip:aliceeatlanta. example.com>; tag=9fxced76sl

To: Bob <sip:boblbiloxi.example.com>

Call-ID: 2xTb9vxSit55XU7p8@atlanta.example.com

CSeq: 1 INVITE

Contact: <sip:alicedclient.atlanta.example.com; transport=tcp>

Content-Type: application/sdp

Content-Length: 151

$\mathrm{V}=0$

o=alice 28908445262890844526 IN IP4 client.atlanta.example.com

$\mathrm{S}=-$

$\mathrm{C}=$ IN IP 4 192.0.2.101

$t=0 \quad 0$

m=audio 49172 RTP/AVP 0

a=rtpmap: 0 PCMU $/ 8000$

F5 100 Trying Proxy $2->$ Proxy 1

SIP/2.0 100 Trying

Via: SIP/2.0/TCP ss1.atlanta.example.com:5060; branch=z9hG4bK2d4790.1 ; received $=192.0 .2 .111$

Via: SIP/2.0/TCP client.atlanta.example.com:5060; branch=z9hG4bK74bf 9 ; received=192.0.2.101

From: Alice <sip:alicelatlanta.example.com>; tag=9fxced76sl

To: Bob <sip:bob@biloxi.example.com>

Call-ID: 2xTb9vxSit55XU7p8@atlanta.example.com

CSeq: 1 INVITE

Content-Length: 0

F6 486 Busy Here Bob $->$ Proxy 2

SIP/2.0 486 Busy Here

Via: SIP/2.0/TCP ss2.biloxi.example.com:5060; branch=z9hG4bK721e4.1 ; received=192.0.2.222

Via: SIP/2.0/TCP ss1.atlanta. example.com:5060; branch=z 9hG4bK2d4790.1 


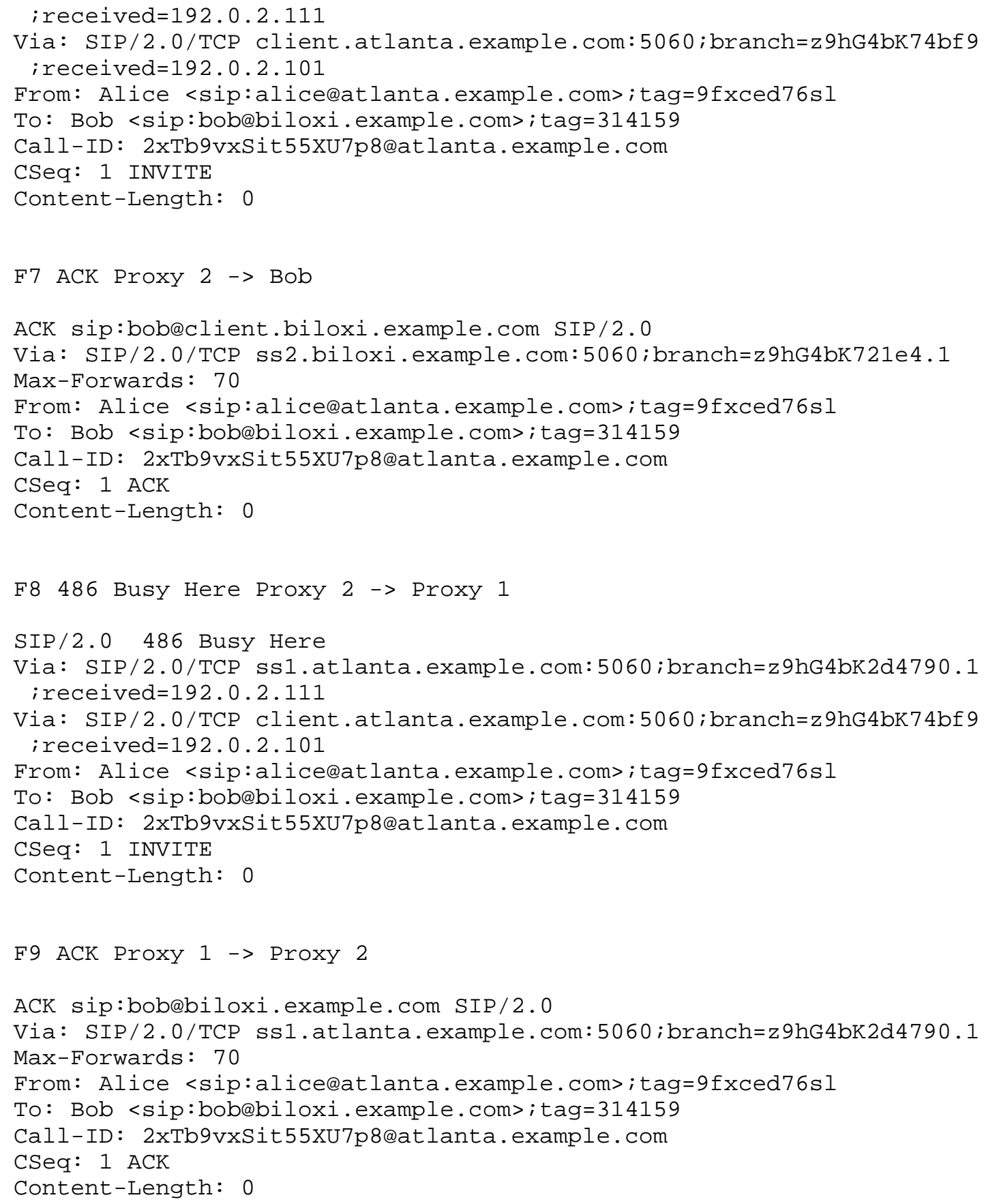




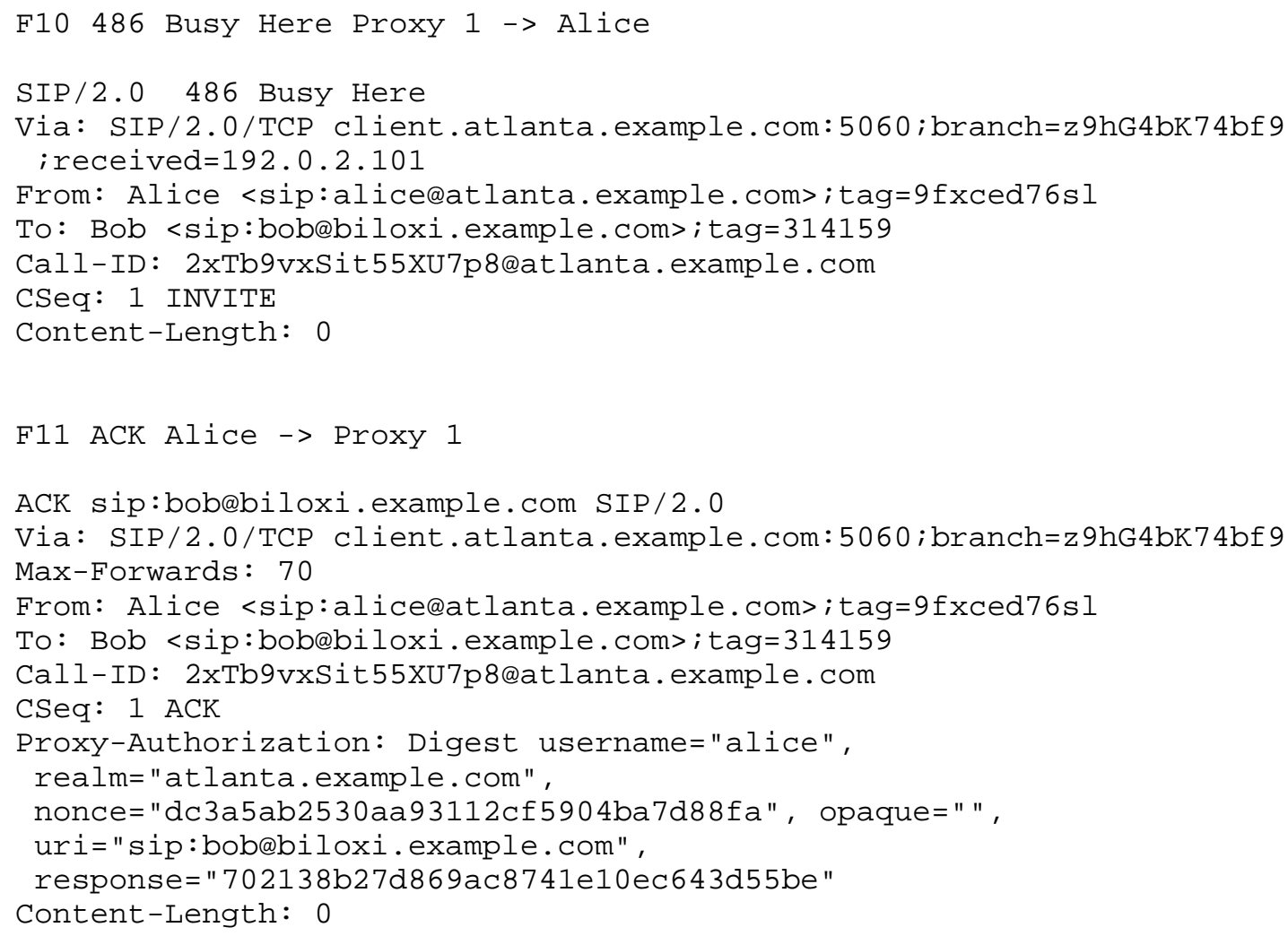


3.10. Unsuccessful No Response from User Agent

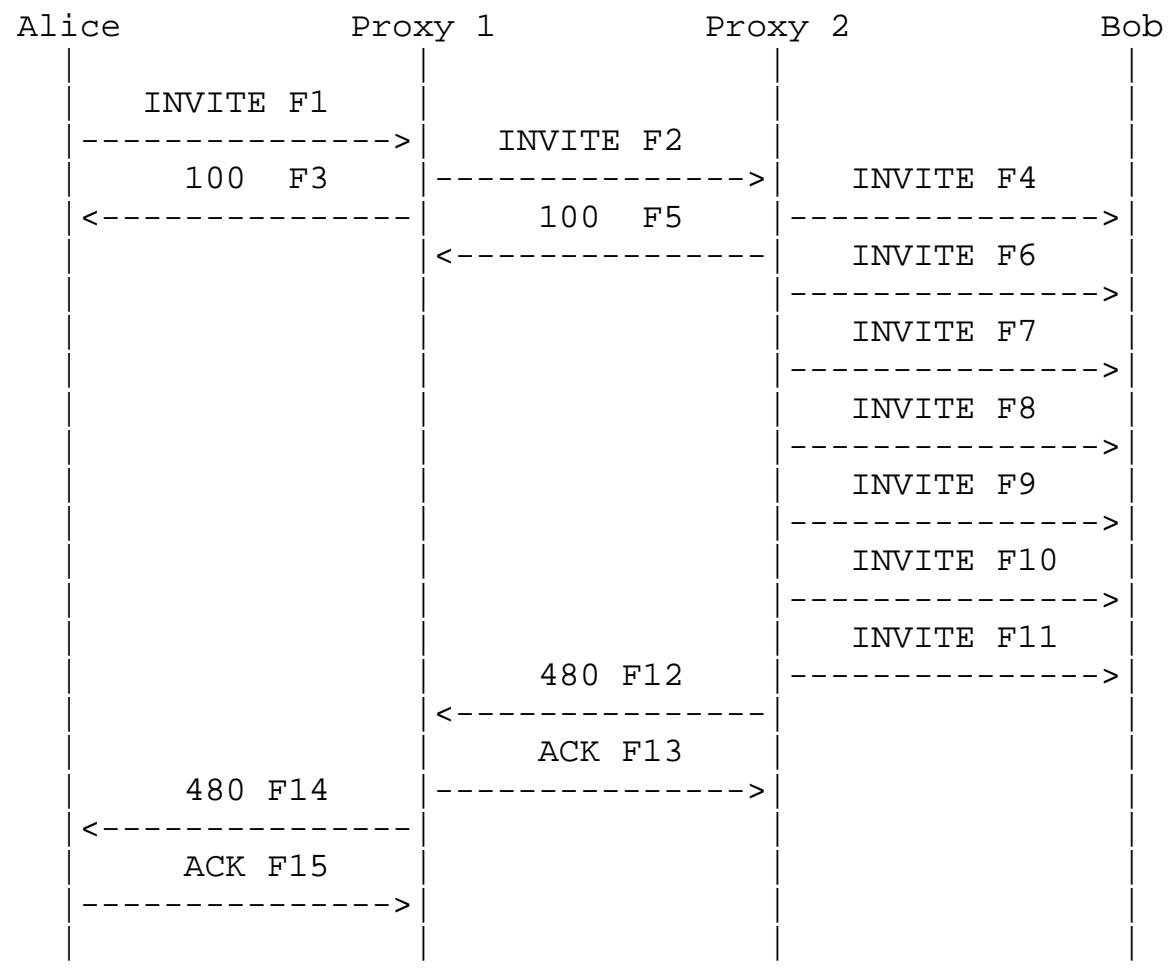

In this example, there is no response from Bob to Alice's INVITE messages being re-transmitted by Proxy 2. After the sixth re-transmission, Proxy 2 gives up and sends a 480 No Response to Alice.

Message Details

F1 INVITE Alice $->$ Proxy 1

INVITE sip:bob@biloxi.example.com SIP/2.0

Via: SIP/2.0/UDP client.atlanta.example.com:5060; branch=z9hG4bK74bf 9 Max-Forwards: 70

Route: <sip:ss1.atlanta.example.com; lr>

From: Alice <sip:alicelatlanta.example.com>; tag=9fxced76sl

To: Bob <sip:bob@biloxi.example.com>

Call-ID: 2xTb9vxSit55XU7p8@atlanta.example.com

CSeq: 1 INVITE

Contact: <sip:alice@client.atlanta.example.com>

Proxy-Authorization: Digest username="alice", realm="atlanta.example.com", 


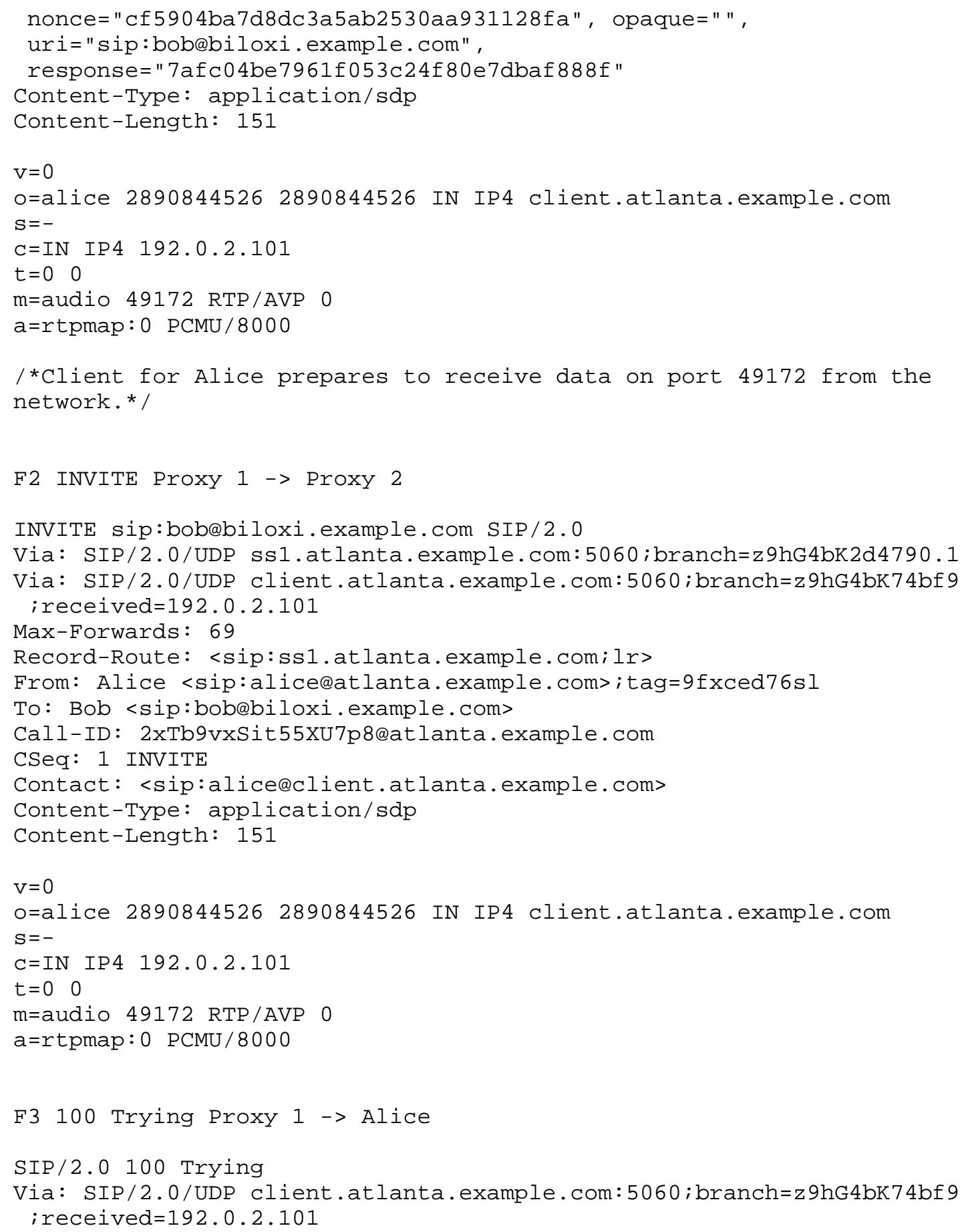




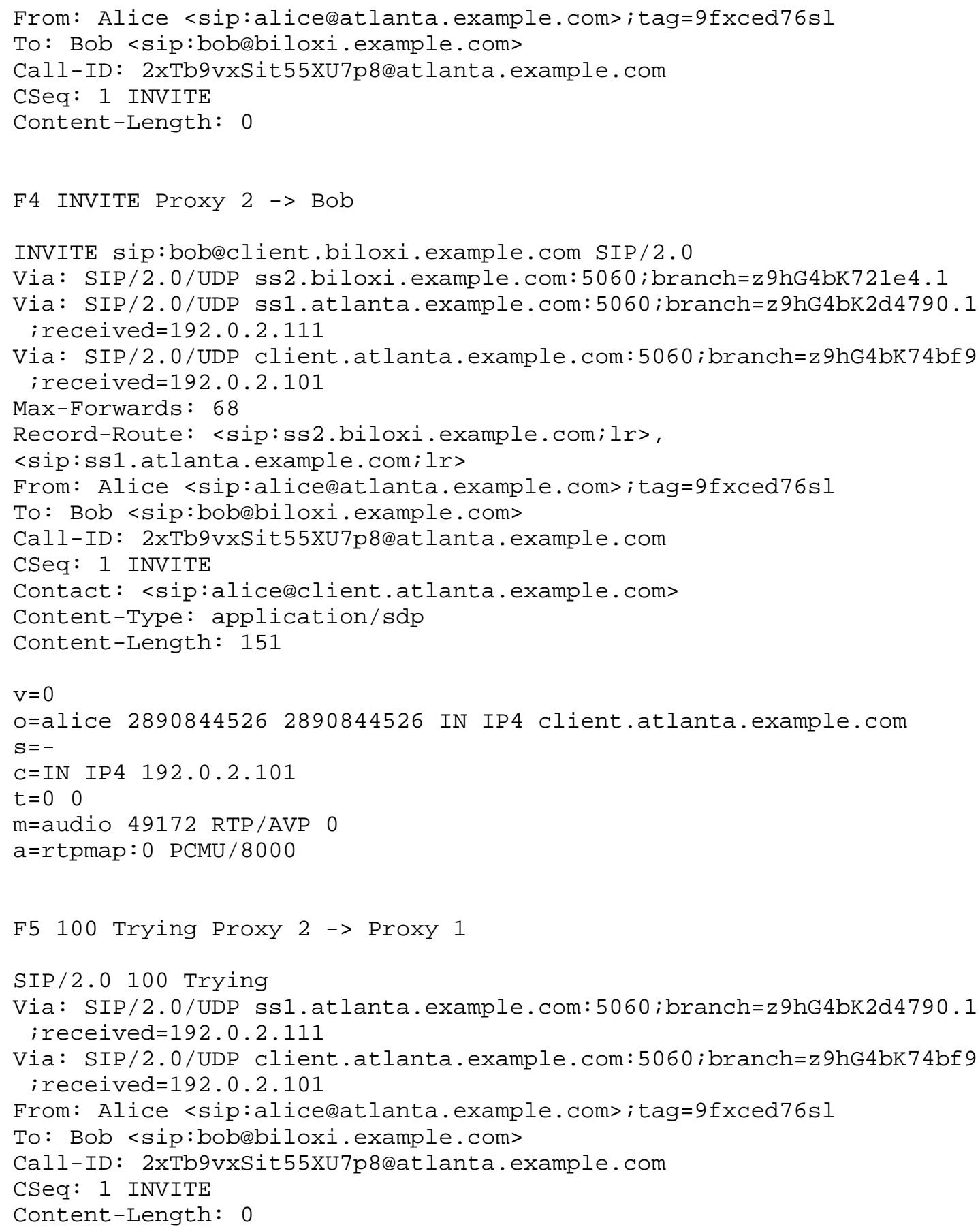


F6 INVITE Proxy $2->$ Bob

Resend of Message F4

F7 INVITE Proxy $2->$ Bob

Resend of Message F4

F8 INVITE Proxy $2 \rightarrow$ Bob

Resend of Message F4

F9 INVITE Proxy $2->$ Bob

Resend of Message F4

F10 INVITE Proxy $2 \rightarrow$ Bob

Resend of Message F4

F11 INVITE Proxy $2->$ Bob

Resend of Message F4

/* Proxy 2 gives up */

F12 480 No Response Proxy $2->$ Proxy 1

SIP/2.0 480 No Response

Via: SIP/2.0/UDP ss1.atlanta. example.com:5060; branch=z 9hG4bK2d4790.1 ; received=192.0.2.111

Via: SIP/2.0/UDP client.atlanta.example.com:5060; branch=z9hG4bK74bf 9 ; received=192.0.2.101

From: Alice <sip:alicelatlanta.example.com>; tag=9fxced76sl

To: Bob <sip:bob@biloxi.example.com>; tag=314159

Call-ID: 2xTb9vxSit55XU7p8@atlanta.example.com

CSeq: 1 INVITE

Content-Length: 0 


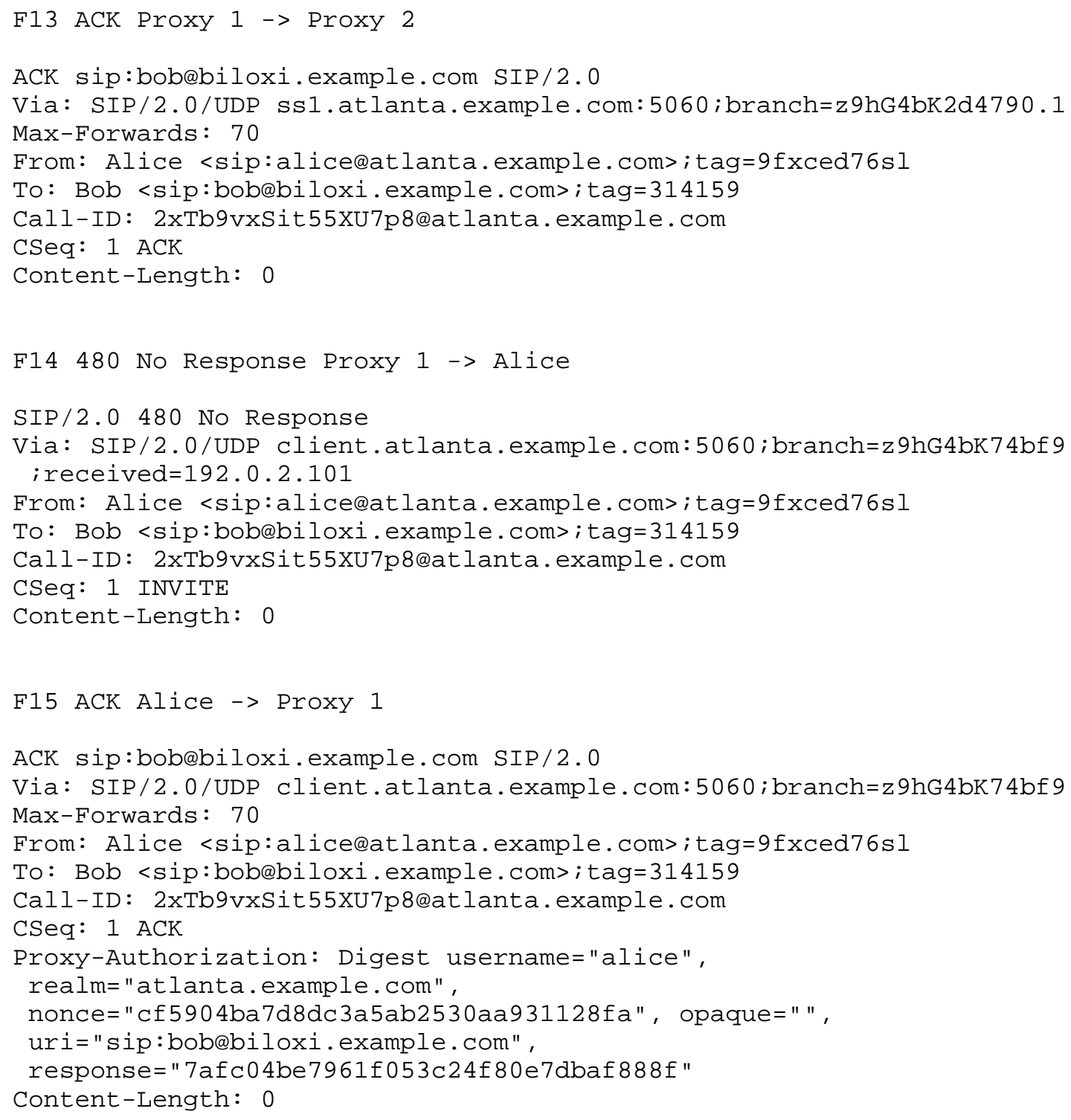


3.11. Unsuccessful Temporarily Unavailable

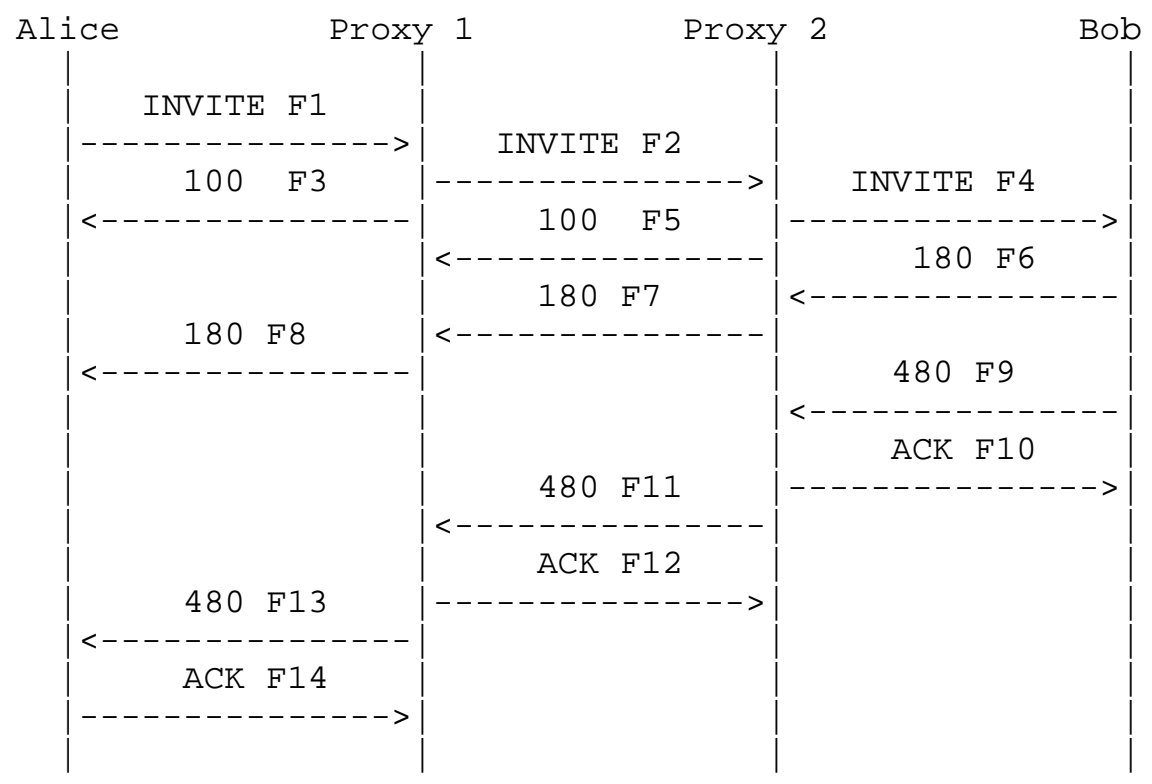

In this scenario, Bob initially sends a 180 Ringing response to Alice, indicating that alerting is taking place. However, then a 480 Unavailable is then sent to Alice. This response is acknowledged then proxied back to Alice.

Message Details

F1 INVITE Alice $->$ Proxy 1

INVITE sip:bob@biloxi.example.com SIP/2.0

Via: SIP/2.0/UDP client.atlanta.example.com:5060; branch=z9hG4bK74bf 9 Max-Forwards: 70

Route: <sip:ssl.atlanta.example.com; lr>

From: Alice <sip:alicelatlanta.example.com>; tag=9fxced76sl

To: Bob <sip:boblbiloxi.example.com>

Call-ID: 2xTb9vxSit55XU7p8@atlanta.example.com

CSeq: 1 INVITE

Contact: <sip:alicedclient.atlanta.example.com>

Proxy-Authorization: Digest username="alice", realm="atlanta.example.com", nonce="aa9311cf5904ba7d8dc3a5ab253028fa", opaque=" ", uri="sip:bob@biloxi . example.com", response="59a46a91bf1646562a4d486c84b399db"

Content-Type: application/sdp 


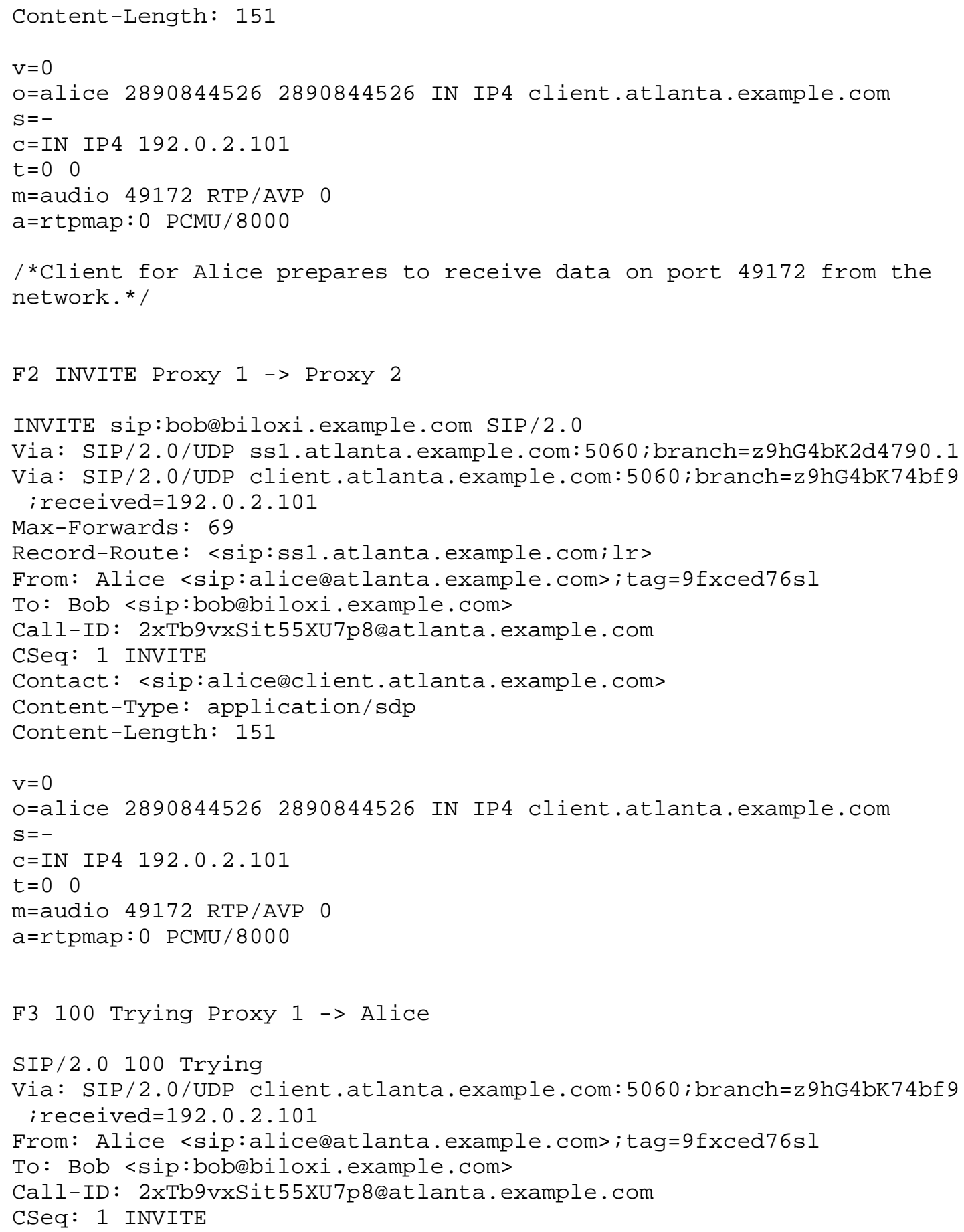




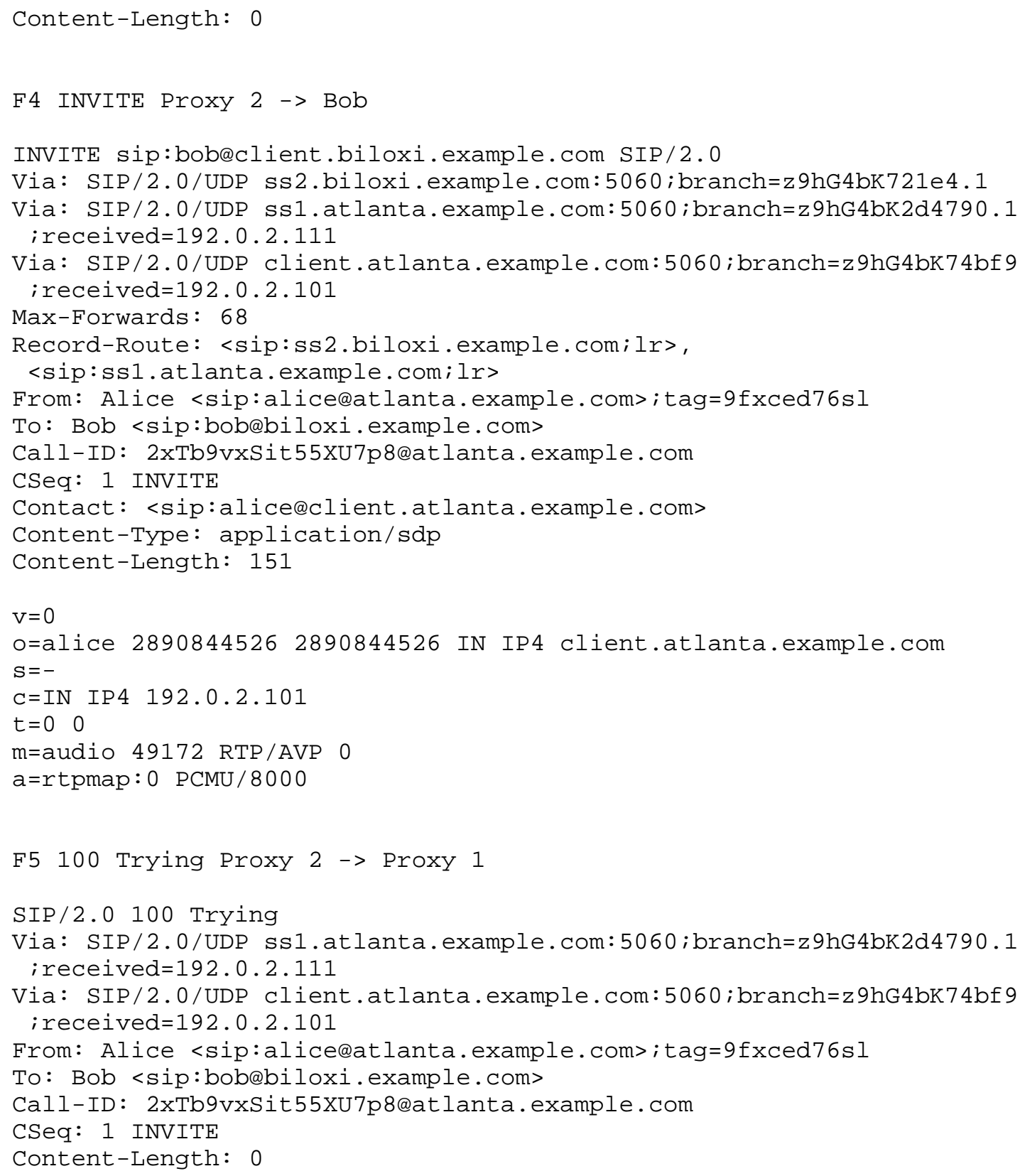




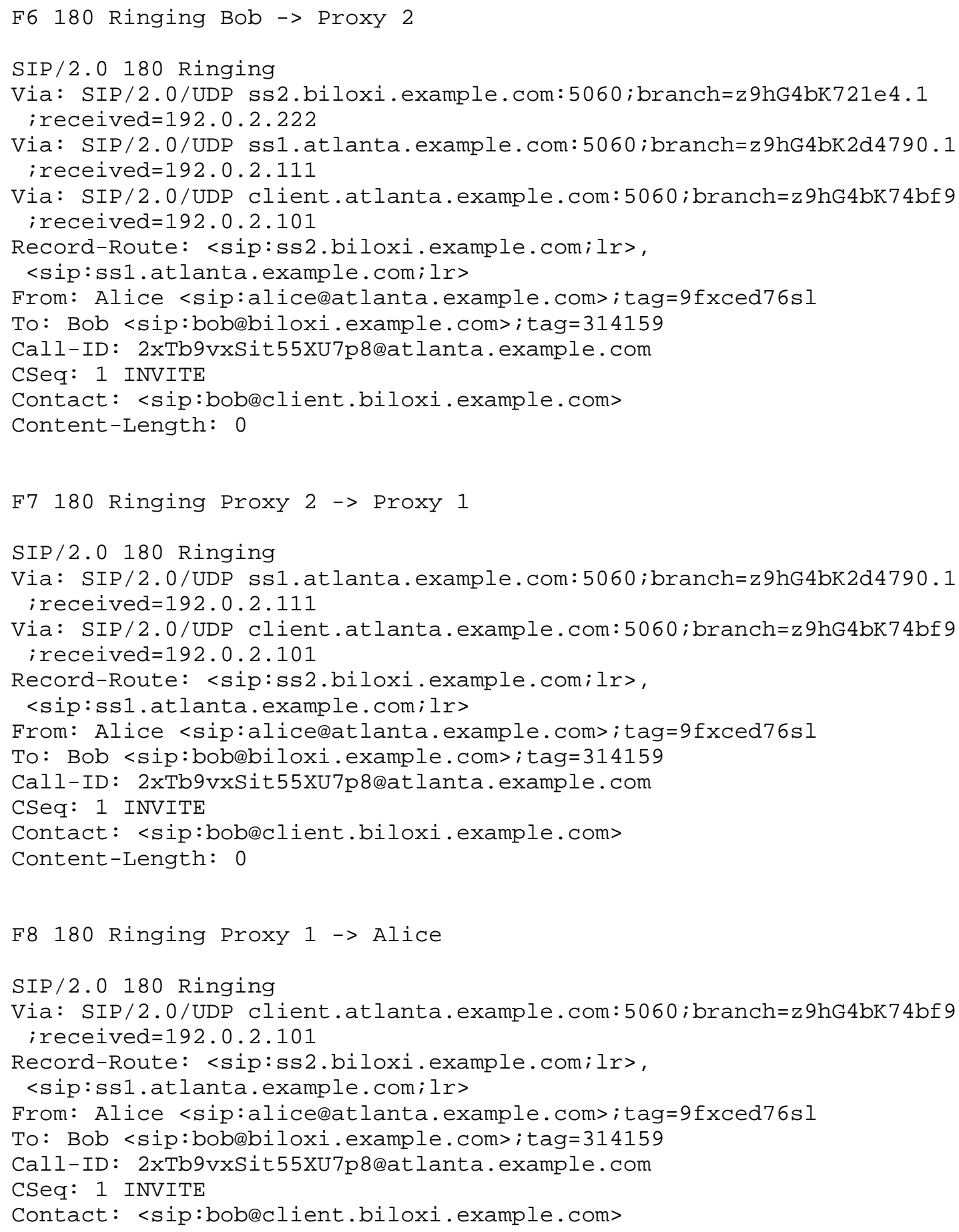




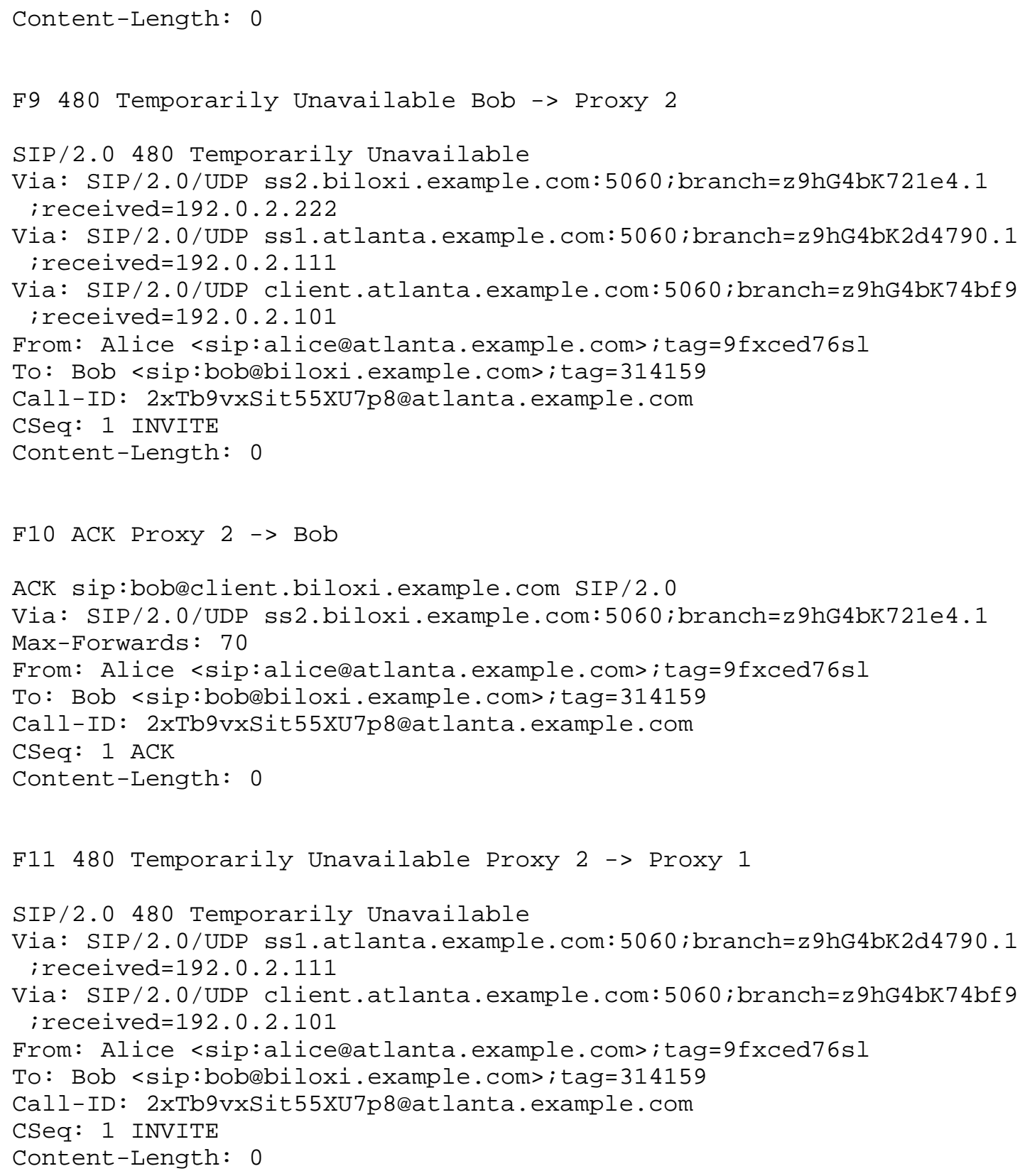




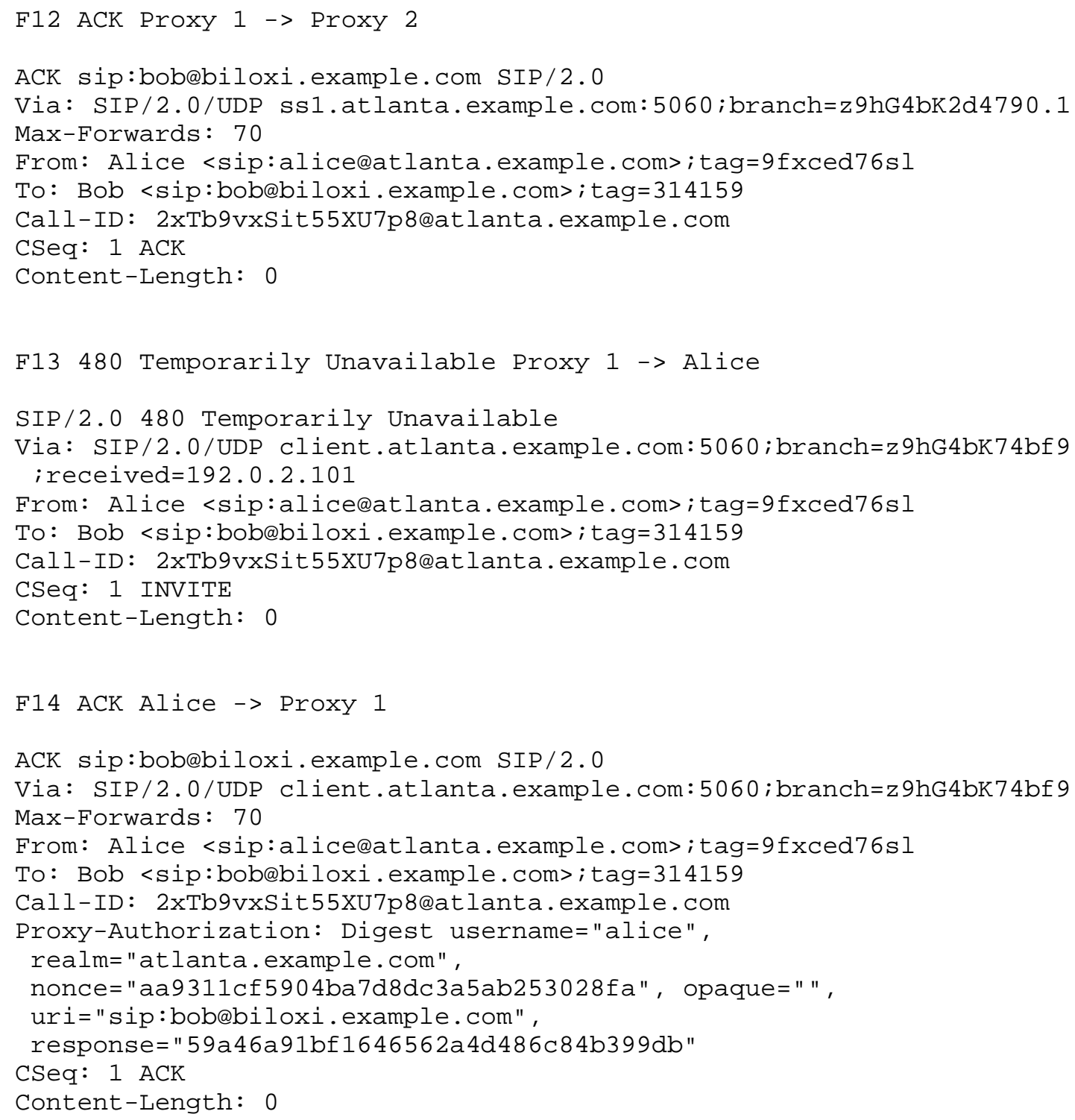




\section{Security Considerations}

Since this document contains examples of SIP session establishment, the security considerations in RFC 3261 [1] apply. RFC 3261 describes the basic threats including registration hijacking, server impersonation, message body tampering, session modifying or teardown, and denial of service and amplification attacks. The use of HTTP Digest as shown in this document provides one-way authentication and protection against replay attacks. TLS transport is used in registration scenarios due to the lack of integrity protection in HTTP Digest and the danger of registration hijacking without it, as described in RFC 3261 [1]. A full discussion of the weaknesses of HTTP Digest is provided in RFC 3261 [1]. The use of TLS and the Secure SIP (sips) URI scheme provides a better level of security including two-way authentication. S/MIME can provide end-to-end confidentiality and integrity protection of message bodies, as described in RFC 3261.

5. References

5.1. Normative References

[1] Rosenberg, J., Schulzrinne, H., Camarillo, G., Johnston, A., Peterson, J., Sparks, R., Handley, M. and E. Schooler, "SIP: Session Initiation Protocol", RFC 3261, June 2002.

[2] Rosenberg, J. and H. Schulzrinne, "An Offer/Answer Model with SDP", RFC 3264, April 2002 .

[3] Franks, J., Hallam-Baker, P., Hostetler, J., Lawrence, S., Leach, P., Luotonen, A. and L. Stewart, "HTTP authentication: Basic and Digest Access Authentication", RFC 2617, June 1999.

[4] Bradner, S., "Key words for use in RFCs to Indicate Requirement Levels", BCP 14, RFC 2119, March 1997.

\subsection{Informative References}

[5] Johnston, A., Donovan, S., Sparks, R., Cunningham, C. and K. Summers, "Session Initiation Protocol (SIP) Public Switched Telephone Network (PSTN) Call Flows", BCP 76, RFC 3666, December 2003.

6. Intellectual Property statement

The IETF takes no position regarding the validity or scope of any intellectual property or other rights that might be claimed to pertain to the implementation or use of the technology described in 
this document or the extent to which any license under such rights might or might not be available; neither does it represent that it has made any effort to identify any such rights. Information on the IETF's procedures with respect to rights in standards-track and standards-related documentation can be found in BCP-11. Copies of claims of rights made available for publication and any assurances of licenses to be made available, or the result of an attempt made to obtain a general license or permission for the use of such proprietary rights by implementors or users of this specification can be obtained from the IETF Secretariat.

The IETF invites any interested party to bring to its attention any copyrights, patents or patent applications, or other proprietary rights which may cover technology that may be required to practice this standard. Please address the information to the IETF Executive Director.

\section{Acknowledgments}

This document is has been a group effort by the SIP and SIPPING WGs. The authors wish to thank everyone who has read, reviewed, commented, or made suggestions to improve this document.

Thanks to Rohan Mahy, Adam Roach, Gonzalo Camarillo, Cullen Jennings, and Tom Taylor for their detailed comments during the final review. Thanks to Dean Willis for his early contributions to the development of this document.

The authors wish to thank Kundan Singh for performing parser validation of messages.

The authors wish to thank the following individuals for their participation in the review of this call flows document: Aseem Agarwal, Rafi Assadi, Ben Campbell, Sunitha Kumar, Jon Peterson, Marc Petit-Huguenin, Vidhi Rastogi, and Bodgey Yin Shaohua.

The authors also wish to thank the following individuals for their assistance: Jean-Francois Mule, Hemant Agrawal, Henry Sinnreich, David Devanatham, Joe Pizzimenti, Matt Cannon, John Hearty, the whole MCI WorldCom IPOP Design team, Scott Orton, Greg Osterhout, Pat Sollee, Doug Weisenberg, Danny Mistry, Steve McKinnon, and Denise Ingram, Denise Caballero, Tom Redman, Ilya Slain, Pat Sollee, John Truetken, and others from MCI WorldCom, 3Com, Cisco, Lucent and Nortel. 


\section{Authors' Addresses}

All listed authors actively contributed large amounts of text to this document.

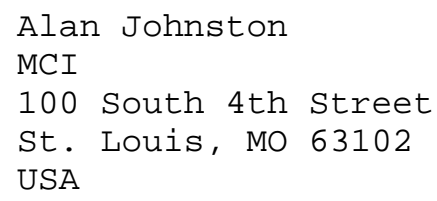


9. Full Copyright statement

Copyright (C) The Internet Society (2003). All Rights Reserved.

This document and translations of it may be copied and furnished to others, and derivative works that comment on or otherwise explain it or assist in its implementation may be prepared, copied, published and distributed, in whole or in part, without restriction of any kind, provided that the above copyright notice and this paragraph are included on all such copies and derivative works. However, this document itself may not be modified in any way, such as by removing the copyright notice or references to the Internet society or other Internet organizations, except as needed for the purpose of developing Internet standards in which case the procedures for copyrights defined in the Internet Standards process must be followed, or as required to translate it into languages other than English.

The limited permissions granted above are perpetual and will not be revoked by the Internet society or its successors or assignees.

This document and the information contained herein is provided on an "AS IS" basis and THE INTERNET SOCIETY AND THE INTERNET ENGINEERING TASK FORCE DISCLAIMS ALL WARRANTIES, EXPRESS OR IMPLIED, INCLUDING BUT NOT LIMITED TO ANY WARRANTY THAT THE USE OF THE INFORMATION HEREIN WILL NOT INFRINGE ANY RIGHTS OR ANY IMPLIED WARRANTIES OF MERCHANTABILITY OR FITNESS FOR A PARTICULAR PURPOSE.

Acknowledgement

Funding for the RFC Editor function is currently provided by the Internet society. 